\title{
Production and Testing of the DNA Few-Group Coupled Neutron-Gamma Cross-Section Library
}
D. E. Bartine
J. R. Knight
J. V. Pace, III
R. Roussin

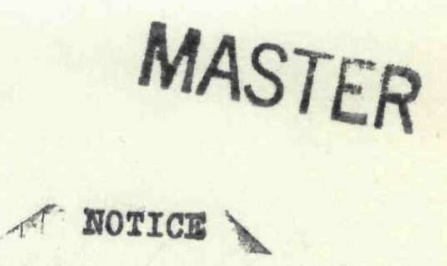

PORTIONS OF THIS REPORT ART IIIEGTBLE. It has been reproduced fros the best ava1lable copy to permit the broadest possible availability.

\section{OAK RIDGE NATIONAL LABORATORY}

OPERATED BY UNION CARBIDE CORPORATION FOR THE ENERGY RESEARCH AND DEVELOPMENT ADMINISTRATION 


\section{Printed in the United States of America. Available from National Technical Information Service}

U.S. Department of Commerce 5285 Port Royal Road, Springfield, Virginia 22161

Price: Printed Copy $\$ 5.50$; Microfiche $\$ 3.00$

This report was prepared as an account of work sponsored by the United States Government. Neither the United States nor the Energy Research and Development Administration/United States Nuclear Regulatory Commission, nor any of their employees, nor any of their contractors, subcontractors, or their employees, makes any warranty, express or implied, or assumes any legal liability or responsibility for the accuracy, completeness or usefulness of any information, apparatus, product or process disclosed, or sepresents that its use would not infringe privately owned rights. 


\section{DISCLAIMER}

This report was prepared as an account of work sponsored by an agency of the United States Government. Neither the United States Government nor any agency Thereof, nor any of their employees, makes any warranty, express or implied, or assumes any legal liability or responsibility for the accuracy, completeness, or usefulness of any information, apparatus, product, or process disclosed, or represents that its use would not infringe privately owned rights. Reference herein to any specific commercial product, process, or service by trade name, trademark, manufacturer, or otherwise does not necessarily constitute or imply its endorsement, recommendation, or favoring by the United States Government or any agency thereof. The views and opinions of authors expressed herein do not necessarily state or reflect those of the United States Government or any agency thereof. 


\section{DISCLAIMER}

Portions of this document may be illegible in electronic image products. Images are produced from the best available original document. 
Contract No. W-7405-eng-26

Neutron Physics Division

\author{
PRODUCTION AND TESTING OF THE DNA FEW-GROUP COUPLED \\ NEUTRON-GAMMA CROSS-SECTION LIBRARY \\ D. E. Bartine, J. R. Knight,* J. V. Pace, III,* \\ and R. Roussin
}

\author{
NOTE : \\ Work Supported by \\ DEFENSE NUCLEAR AGENCY \\ under \\ Subtask PC 103
}

Date Published: March 1977

*Computer Sciences Division

\author{
OAK RIDGE NATIONAL LABORATORY \\ Oak Ridge, Tennessee 37830 \\ operated by \\ UNION CARBIDE CORPORATION \\ for the \\ ENERGY RESEARCH AND DEVELOPMENT ADMINTSTRATION
}

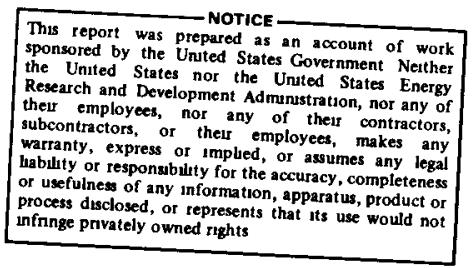


$\bullet$

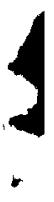

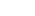

' 
Abstract .................... . vii

I. Introduction . . . . . . . . . . . . . 1

II. Libnary Contents . . . . . . . . . . . . 2

III. Sources and Responses . . . . . . . . . . 6

IV. Testing ................. 13

References . . . . . . . . . . . . 19

Appendix A: Listing of Partial Cross Sections . . . . Al

Appendix B: Listing of Response Functions . . . . . B1

Appendix C: ARID - A Simple Collapsing Code . . . . . C1 


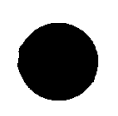

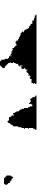


ACKNOWLEDGMENTS

The authors are especially indebted to R. Q. Wright for his patient efforts in processing the trial group structures leading up to the adoption of the 37-21 structure, and for processing the initial library. The authors are also indebted to W. E. Ford, III, to D. M. Plaster, and to $C$. C. Webster for generating subsequent cross-section sets. Special thanks is due to N. M. Greene for his assistance in debugging the crosssection sets and for his contribution of the ARID module. The project itself is the result of the foresight and planning of F. R. Mynatt of ORNL and D. Kau1 of SAI, former1y Project Officer at DNA. The existence of this manual is due to the organizational skills and typing of $G$. B. Raiford, which is deeply appreciated. 
•

4 


\begin{abstract}
A state-of-the-art cross-section library has been developed for the Defense Nuclear Agency in a 37-21 neutron-gamma energy group structure that can be used for radiation transport calculations on most available computer facilities. Based on data from the DNA Working Cross Section Library and the Evaluated Nuclear Data File, the library contains cross sections for 35 nuclides that will be updated and added to regularly. The documentation presented here includes descriptions of typical sources and responses in the 37-21 structure and results of comparative calculations performed for testing purposes. The library is available through the Radiation Shielding Information Center at Oak Ridge National Laboratory.
\end{abstract}




\section{INTRODUCTION}

Many contractors performing radiation transport calculations for the Defense Nuclear Agency do not have access to the large computer facilities required to use the detailed sets of coupled neutron and gamma-ray interaction cross sections developed by the several shielding research organizations. To aid their contractors, DNA requested that ORNL develop and test a coupled cross-section library having the minimum number of neutron and gamma-ray energy groups to obtain acceptable accuracy in the transport results. The resulting library, processed with the AMPX code, ${ }^{1}$ contains 37 neutron groups and 21 gamma-ray groups and includes cross sections for 35 nuclides of specific interest to the defense community. The library is based on cross-section data from the DNA Working Cross Section Library (DNA WCSL)* and the Evaluated Nuclear Data File. ${ }^{2}$ As new data become available from these two sources, the library will be subject to regular maintenance and revision and thereby will represent the state of the art.

This report presents the documentation of the library. Section II gives a listing of the contents of the library in terms of nuclides, with appropriate references. It also presents the energy group structure, along with details regarding the processing employed.

Section III contains a listing of the response functions included in the library and a discussion of their application, together with descriptions of frequently employed source distributions. Calculations performed to test the library by comparison with other libraries are described in

\footnotetext{
*R. W. Roussin, "The Defense Nuclear Agency Working Cross Section Library: Description and Contents," ORNL-RSIC-34 (1972) Revised 1973. This document is periodically revised to reflect changes in the DNA WCSL, but a more timely status is given by means of the RSIC Monthly Newsletter.
} 
Section IV. Complete problem descriptions are given, as well as the results obtained and conclusions drawn, particularly with regard to group structure and weighting functions.

Appendix A contains a ligting of the partial reaction cross sections (for example, elastic, inelastic, etc.) by element, and describes a simple module for collapsing the 37-21 neutron-gamma group library to fewer energy groups, as in a 22-18 neutron-gamma structure.

Inquiries or requests for the library package may be mailed to:

Data Coordinator

Radiation Shielding Information Center (RSIC)

Oak Ridge National Laboratory

Post Office Box X

Oak Ridge, Tennessee 37830 .

The library is designated DLC-31/(DPL-1/FEWGI) by RSIC.

\section{LIBRARY CONTENTS}

A list of the 35 nuclides included in this cross-section library is presented in Table 1, along with the names of the evaluators, the date of the evaluation, and the data set identification in the DNA Working Cross Section Library and/or Evaluated Nuclear Data File (ENDF/B).

As stated previously, the cross sections were processed into a coupled 37-21 neutron-gamma energy group structure, with gamma-ray production included for each element. The energy structure, shown in Table 2, is quite biased at high energies for both the neutron and the gamma-ray groups owing to the importance of high-energy particles for the calculation of tissue dose and silicon damage in electronics. ${ }^{3}$ In addition, the neutron groups are tailored to allow for the major peaks and valleys in the total neutron cross sections of nitrogen, oxygen, silicon, and iron, and the gamma-ray groups are tailored to allow 
Table 1. DATA DESCRIPTION FOR THE DEFENSE NUCLEAR AGENCY FEW-GROUP CROSS-SECTION LIBRARY

\begin{tabular}{|c|c|c|c|c|}
\hline Materia1 & Evaluators & Date & $\begin{array}{c}\text { DNA } \\
\text { MAT-MOD } \\
\end{array}$ & $\begin{array}{l}\text { ENDF/B-IV } \\
\text { Equivalent }\end{array}$ \\
\hline H & Stewart, LaBauve, Young - LASL & $10 / 73$ & $4148-2$ & 1269 \\
\hline $\mathrm{H}-3$ & Stewart - LASL & $10 / 73$ & $4169-1$ & 1169 \\
\hline $\mathrm{Li}-6$ & Battat, LaBauve - LASL & 1974 & & 1271 \\
\hline $\mathrm{Li}-7$ & Battat, LaBauve - LASL & 1974 & & 1272 \\
\hline $\mathrm{Be}-9$ & Howerton, Perkins - LLL & 1973 & $4154-3$ & 1289 \\
\hline$B-10$ & LaBauve, Young, Hale - LASL & 1972 & & $1159 *$ \\
\hline $\mathrm{B}-11$ & LaBauve, Young, Hale - LASL & 1973 & & $1160 *$ \\
\hline$C-12$ & Perey, Perey - ORNL & $2 / 74$ & 4274-0 & 1274 \\
\hline $\mathrm{N}$ & Young, Foster - LASL & $7 / 73$ & $4133-4$ & 1275 \\
\hline 0 & Young, Foster - LASL & $8 / 73$ & $4134-2$ & 1276 \\
\hline $\mathrm{F}$ & Fu, Larson, Perey - ORNL & $9 / 74$ & $4509-1$ & 1277 \\
\hline $\mathrm{Na}$ & Paik, Pitterle, Perey - WARD, ORNL & $2 / 72$ & $4156-0$ & 1156 \\
\hline Mg & Drake, Fricke - SAI & $2 / 74$ & 4512-1 & 1280 \\
\hline A1 & Foster, Young - LASL & $11 / 73$ & $4135-3$ & 1193 \\
\hline $\begin{array}{l}\mathrm{Si} \\
\mathrm{CI}\end{array}$ & $\begin{array}{l}\text { Larson, Perey, Drake, Young - ORNL } \\
\text { Drake - GGA }\end{array}$ & $\begin{array}{l}2 / 74 \\
1967\end{array}$ & $4151-3$ & $\begin{array}{l}1194 \\
1149\end{array}$ \\
\hline $\mathrm{K}$ & Drake - GGA & 1967 & & 1150 \\
\hline $\mathrm{Ca}$ & Fu, Perey - ORNL & $2 / 73$ & $4152-3$ & 1195 \\
\hline $\mathrm{V}$ & Penny, Owen - ORNL & $9 / 72$ & & 1196 \\
\hline $\mathrm{Cr}$ & Prince - BNL & $4 / 74$ & & 1191 \\
\hline Mn & Takahashi - BNL & $2 / 74$ & & 1197 \\
\hline $\mathrm{Fe}$ & Perey, Fu, Penny, Kinney, Wright - ORNL & $2 / 74$ & $4180-2$ & 1192 \\
\hline $\mathrm{Ni}$ & Bhat - BNL & $12 / 73$ & & 1190 \\
\hline $\mathrm{Cu}$ & Drake, Fricke - SAI & $2 / 74$ & $4529-1$ & 1295 \\
\hline Mo & Howerton - LLL & $9 / 74$ & & 1287 \\
\hline $\begin{array}{l}\mathrm{Ta}-181 \\
\mathrm{~W}-182\end{array}$ & $\begin{array}{l}\text { Howerton, Perkins, MacGregor - LLL } \\
\text { Young - LASL }\end{array}$ & $\begin{array}{r}10 / 73 \\
3 / 74\end{array}$ & $\begin{array}{l}4179-3 \\
4582-2\end{array}$ & $\begin{array}{l}1285 \\
1128\end{array}$ \\
\hline W-183 & Young - LASL & $3 / 74$ & 4583-2 & 1129 \\
\hline$W-184$ & Young - LASL & $3 / 74$ & $4584-2$ & 1130 \\
\hline W-186 & Young - LASL & $3 / 74$ & $4586-2$ & 1131 \\
\hline $\mathrm{Pb}$ & Fu, Perey - ORNL & $10 / 73$ & $4136-5$ & 1288 \\
\hline $\mathrm{U}-235$ & Howerton, MacGregor - LLL & $3 / 73$ & $4188-1$ & \\
\hline $\mathrm{U}-238$ & Howerton, MacGregor - LLL & $3 / 73$ & $4187-1$ & \\
\hline $\mathrm{Pu}-239$ & Stewart, Hunter - LASL & $3 / 73$ & $4539-0$ & \\
\hline $\mathrm{Pu}-240$ & Stewart, Hunter - LASL & $3 / 73$ & $4540-0$ & \\
\hline
\end{tabular}

* Secondary gamma-ray production cross sections processed with POPOP4. 
Table 2. NEUTRON AND GAMMA-RAY ENERGY BOUNDARIES FOR THE 37-21 COUPLED NEUTRON-GAMMA LIBRARY

\begin{tabular}{|c|c|c|c|c|}
\hline \multirow[b]{2}{*}{ Group No. } & \multicolumn{2}{|c|}{ Neutron Group (eV) } & \multicolumn{2}{|c|}{ Gamma Group (eV) } \\
\hline & Energy & Lethargy & Energy & Eff. Avg. \\
\hline 1 & $1.96+7 *$ & -0.675 & $1.40+7$ & $1.20+7$ \\
\hline 2 & $1.69+7$ & -0.525 & $1.00+7$ & $9.00+6$ \\
\hline 3 & $1.49+7$ & -0.400 & $8.00+6$ & $7.50+6$ \\
\hline 4 & $1.42+7$ & -0.350 & $7.00+6$ & $6.50+6$ \\
\hline 5 & $1.38+7$ & -0.325 & $6.00+6$ & $5.50+6$ \\
\hline 6 & 1. $28+7$ & -0.250 & $5.00+6$ & $4.50+6$ \\
\hline 7 & $1.22+7$ & -0.200 & $4.00+6$ & $3.50+6$ \\
\hline 8 & $1.11+7$ & -0.100 & $3.00+6$ & $2.75+6$ \\
\hline 9 & $1.00+7$ & 0.000 & $2.50+6$ & $2.25+6$ \\
\hline 10 & $9.05+6$ & 0.100 & $2.00+6$ & $1.75+6$ \\
\hline 11 & $8.19+6$ & 0.200 & 1. $50+6$ & $1.25+6$ \\
\hline 12 & $7.41+6$ & 0.300 & 1. $00+6$ & $8.50+5$ \\
\hline 13 & $6.38+6$ & 0.450 & $7.00+5$ & $5.75+5$ \\
\hline 14 & $4.97+6$ & 0.700 & $4.50+5$ & $3.75+5$ \\
\hline 15 & $4.72+6$ & 0.750 & $3.00+5$ & $2.25+5$ \\
\hline 16 & $4.07+6$ & 0.900 & $1.50+5$ & $1.25+5$ \\
\hline 17 & $3.01+6$ & 1.200 & $1.00+5$ & $8.50+4$ \\
\hline 18 & $2.39+6$ & 1.433 & $7.00+4$ & $5.75+4$ \\
\hline 19 & $2.31+6$ & 1.467 & $4.50+4$ & $3.75+4$ \\
\hline 20 & $1.83+6$ & 1.700 & $3.00+4$ & $2.50+4$ \\
\hline 21 & $1.11+6$ & 2.200 & $2.00+4$ & $1.50+4$ \\
\hline 22 & $5.50+5$ & 2.900 & $1.00+4$ & \\
\hline 23 & 1. $58+5$ & 4.150 & & \\
\hline 24 & $1.11+5$ & 4.500 & & \\
\hline 25 & $5.25+4$ & 5.250 & & \\
\hline 26 & $2.48+4$ & 6.000 & & \\
\hline 27 & $2.19+4$ & 6.125 & & \\
\hline 28 & $1.03+4$ & 6.875 & & \\
\hline 29 & $3.35+3$ & 8.000 & & \\
\hline 30 & $1.23+3$ & 9.000 & & \\
\hline 31 & $5.83+2$ & 9.750 & & \\
\hline 32 & $1.01+2$ & 11.500 & & \\
\hline 33 & $2.90+1$ & 12.750 & & \\
\hline 34 & $1.07+1$ & 13.750 & & \\
\hline 35 & $3.06+0$ & 15.000 & & \\
\hline 36 & $1.13+0$ & 16.000 & & \\
\hline 37 & $4.14-1$ & 17.000 & & \\
\hline 38 & $1.00-5$ & 27.631 & & \\
\hline
\end{tabular}

$*_{\text {Read as }} 1.96 \times 10^{7}$. 
accurate calculation of pair production, annihilation photon transport, hydrogen capture, and backscatter photon transport. There is also sufficient low photon energy structure to allow for rapidly changing cross sections and response functions. The cross-section data were processed with the $\mathrm{AMPX}^{1}$ code, using a $1 / \mathrm{E}$ weighting spectrum for all neutron groups except the thermal group, for which a $300^{\circ} \mathrm{K}$ Maxwellian weighting spectrum was used. $\cdot \mathrm{A}_{3}$ Legendre expansion is given for each element in the $\operatorname{ANISN}^{4}$ format, as follows:

\section{For Energy Group g}

\section{Table Position}

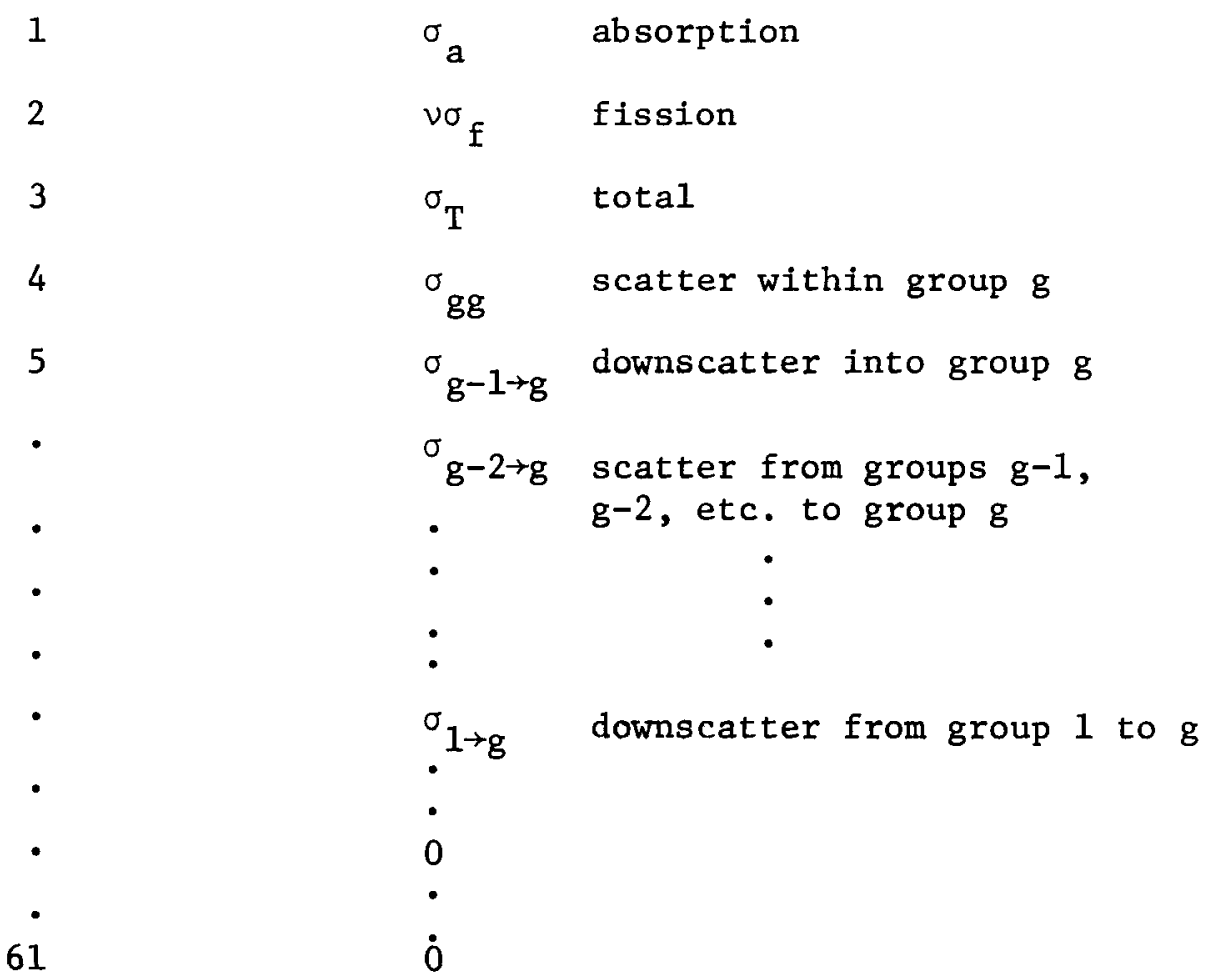

The table length of the cross-section set is therefore 61 to allow for downscatter and production from all higher groups to group 58 (gamma-ray group 21), and the cross sections basically fill in the upper diagonal matrix below the first three positions. 
III. SOURCES AND RESPONSES

This section presents the sources and response functions included with the library for use in defense-related calculations. The titles of the response functions are listed in Table 3, along with the applicable units. Responses 1 and 2, the neutron and gamma free-in-air tissue kerma response functions, were generated for an 11-element standard man ${ }^{5}$ composition from the same data used for the cross-section library with the MACK $^{6}$ code. Free-in-air tissue kerma is also referred to as a single-collision or first-collision dose. Responses 3, 4, and 5 are essentially electronic damage functions. Responses 6 and 7 are neutron and gamma tissue dose responses, respectively. These tissue dose responses were obtained by transporting monoenergetic neutron and gamma broad-beam sources through tissue phantoms and scoring the maximum energy deposition (kerma) in the phantoms. Response 6 , the Snyder-Auxier neutron tissue dose function, ${ }^{7}$ considered a threedimensional homogeneous cylindrical tissue phantom and includes the effects of secondary gammas produced in the phantom. Response 7 , the Claiborne-Trubey gamma tissue dose function, 8 considered a onedimensional homogeneous slab tissue phantom. Table 4 presents the free-in-air kerma functions (responses 1 and 2), the tissue dose (inphantom) functions (responses 6 and 7), and includes the older Henderson neutron free-in-air kerma function for comparison. The entire set of response functions listed in Table 3 are presented in cross-section format as Appendix B. Responses 8 through 21 and 22 through 35 are neutron and gamma elemental kerma factors, respectively. Kerma is 
Table 3. RESPONSES INCLUDED WITH THE 37-21 LIBRARY

\begin{tabular}{|c|c|c|}
\hline Position & Response Function & Units \\
\hline 1 & Neutron Free-in-Air Tissue Kerma & Rads/(Neutron $/ \mathrm{cm}^{2}$ ) \\
\hline 2 & Gamma Free-in-Air Tissue Kerma & Rads/(Neutron $\left./ \mathrm{cm}^{2}\right)$ \\
\hline 3 & Neutron Displacement in Silicon & $\begin{array}{l}\left.\text { (Equiv. } 1-\mathrm{MeV} \text { Neutrons } /\left(\mathrm{cm}^{2} \cdot \mathrm{sec}\right)\right) / \\
\text { Neutron } /\left(\mathrm{cm}^{2} \cdot \mathrm{sec}\right)\end{array}$ \\
\hline 4 & Neutron Ionization in Silicon & (Rads-Si)/(Neutron $\left./ \mathrm{cm}^{2}\right)$ \\
\hline 5 & Gamma Ionization in Silicon & $\left(\right.$ Rads-Si)/(Photon $\left./ \mathrm{cm}^{2}\right)$ \\
\hline 6 & Snyder-Auxier Neutron Tissue Dose & Rads/(Neutron/ $/ \mathrm{cm}^{2}$ ) \\
\hline 7 & Claiborne-Trubey Gamma Tissue Dose & Rads/(Photon $\left./ \mathrm{cm}^{2}\right)$ \\
\hline 8 & H Neutron Kerma & $\left(\right.$ Rads/Atom) $/\left(\right.$ Neutron $\left./\left(\mathrm{cm}^{2} \cdot \mathrm{g}\right)\right)$ \\
\hline 9 & C Neutron Kerma & (Rads/Atom)/(Neutron/ $\left.\left(\mathrm{cm}^{2} \cdot \mathrm{g}\right)\right)$ \\
\hline 10 & N Neutron Kerma & (Rads/Atom)/(Neutron/ $\left.\left(\mathrm{cm}^{2} \cdot \mathrm{g}\right)\right)$ \\
\hline 11 & o Neutron Kerma & (Rads/Atom)/(Neutron/ $\left.\left(\mathrm{cm}^{2} \cdot \mathrm{g}\right)\right)$ \\
\hline 12 & $\mathrm{Na}$ Neutron Kerma & (Rads/Atom)/(Neutron/ $\left.\left(\mathrm{cm}^{2} \cdot \mathrm{g}\right)\right)$ \\
\hline 13 & Mg Neutron Kerma & $(\operatorname{Rads} /$ Atom $) /\left(\right.$ Neutron $\left./\left(\mathrm{cm}^{2} \cdot \mathrm{g}\right)\right)$ \\
\hline 14 & A1 Neutron Kerma & (Rads/Atom)/(Neutron/ $\left.\left(\mathrm{cm}^{2} \cdot \mathrm{g}\right)\right)$ \\
\hline 15 & Si Neutron Kerma & $(\operatorname{Rads} /$ Atom $) /\left(\right.$ Neutron $\left./\left(\mathrm{cm}^{2} \cdot \mathrm{g}\right)\right)$ \\
\hline 16 & $P$ Neutron Kerma & $(\operatorname{Rad} /$ Atom $) /\left(\right.$ Neutron $\left./\left(\mathrm{cm}^{2} \cdot \mathrm{g}\right)\right)$ \\
\hline 17 & S Neutron Kerma & $(\operatorname{Rad} /$ Atom $) /\left(\right.$ Neutron $\left./\left(\mathrm{cm}^{2} \cdot \mathrm{g}\right)\right)$ \\
\hline 18 & C1 Neutron Kerma & $(\operatorname{Rad} \mathrm{s} /$ Atom $) /\left(\right.$ Neutron/ $\left.\left(\mathrm{cm}^{2} \cdot \mathrm{g}\right)\right)$ \\
\hline 19 & $\mathrm{~K}$ Neutron Kerma & (Rads/Atom)/(Neutron/ $\left.\left(\mathrm{cm}^{2} \cdot \mathrm{g}\right)\right)$ \\
\hline 20 & Ca Neutron Kerma & (Rads/Atom)/(Neutron/ $\left.\left(\mathrm{cm}^{2} \cdot \mathrm{g}\right)\right)$ \\
\hline 21 & Fe Neutron Kerma & $\left(\right.$ Rads/Atom) $/\left(\right.$ Neutron $\left./\left(\mathrm{cm}^{2} \cdot \mathrm{g}\right)\right)$ \\
\hline 22 & H Gamma Kerma & $(\operatorname{Rads} /$ Atom $) /\left(\right.$ Photon $\left./\left(\mathrm{cm}^{2} \cdot g\right)\right)$ \\
\hline 23 & C Gamma Kerma & (Rads/Atom) $/\left(\right.$ Photon $\left./\left(\mathrm{cm}^{2} \cdot \mathrm{g}\right)\right)$ \\
\hline 24 & N Gamma Kerma & (Rads/Atom) $/\left(\right.$ Photon $\left./\left(\mathrm{cm}^{2} \cdot \mathrm{g}\right)\right)$ \\
\hline 25 & o Gamma Kerma & (Rads/Atom)/(Photon/ $\left.\left(\mathrm{cm}^{2} \cdot \mathrm{g}\right)\right)$ \\
\hline 26 & Na Gamma Kerma & (Rads/Atom)/(Photon/ $\left.\left(\mathrm{cm}^{2} \cdot \mathrm{g}\right)\right)$ \\
\hline 27 & Mg Gamma Kerma & (Rads/Atom)/(Photon/ $\left.\left(\mathrm{cm}^{2} \cdot \mathrm{g}\right)\right)$ \\
\hline 28 & A1 Gamma Kerma & (Rads/Atom) /(Photon/ $\left.\left(\mathrm{cm}^{2} \cdot \mathrm{g}\right)\right)$ \\
\hline 29 & Si Gamma Kerma & (Rads/Atom)/(Photon/ $\left.\left(\mathrm{cm}^{2} \cdot \mathrm{g}\right)\right)$ \\
\hline 30 & P Gamma Kerma & (Rads/Atom)/(Photon/ $\left.\left(\mathrm{cm}^{2} \cdot \mathrm{g}\right)\right)$ \\
\hline 31 & S Gamma Kerma & (Rads/Atom)/(Photon/ $\left.\left(\mathrm{cm}^{2} \cdot \mathrm{g}\right)\right)$ \\
\hline 32 & C1 Garma Kerma & (Rads/Atom)/(Photon/ $\left.\left(\mathrm{cm}^{2} \cdot \mathrm{g}\right)\right)$ \\
\hline 33 & K Gamma Kerma & (Rads/Atom) $/\left(\right.$ Photon $\left./\left(\mathrm{cm}^{2} \cdot \mathrm{g}\right)\right)$ \\
\hline 34 & Ca Gamma Kerma & (Rads/Atom) /(Photon/ $\left.\left(\mathrm{cm}^{2} \cdot \mathrm{g}\right)\right)$ \\
\hline 35 & Fe Gamma Kerma & (Rads/Atom)/(Photon/ $\left.\left(\mathrm{cm}^{2} \cdot \mathrm{g}\right)\right)$ \\
\hline 36 & Snyder-Neufeld Neutron Tissue Dose & Rads/(Neutron $/ \mathrm{cm}^{2}$ ) \\
\hline 37 & Henderson Neutron Free-in-Air & \\
\hline & Tissue Kerma & Rads/ (Neutron $/ \mathrm{cm}^{2}$ ) \\
\hline 38 & Henderson Gamma Free-in-Air & \\
\hline 39 & Snyder-Auxier Neutron Tissue Dose & Rem/ (Neutron $/ \mathrm{cm}^{2}$ ) \\
\hline
\end{tabular}


Table 4. SELECTED TISSUE RESPONSE FUNCTIONS 4.A. Neutron Responses in Rads/(Neutron $/ \mathrm{cm}^{2}$ )

\begin{tabular}{|c|c|c|c|c|}
\hline Group & $\begin{array}{l}\text { Upper Energy } \\
\text { Boundary (MeV) }\end{array}$ & $\begin{array}{l}\text { Neutron Free-in-Air } \\
\text { Tissue Kerma }\end{array}$ & $\begin{array}{c}\text { Snyder-Auxier } \\
\text { Neutron Tissue } \\
\text { Dose } \\
\end{array}$ & $\begin{array}{c}\text { Henderson } \\
\text { Free-in-Air } \\
\text { Tissue Kerma } \\
\end{array}$ \\
\hline 1 & 19.6 & $7.00-09$ & $7.85-09$ & $5.64-09$ \\
\hline 2 & 16.9 & $6.64-09$ & $7.78-09$ & $5.64-09$ \\
\hline 3 & 14.9 & $6.43-09$ & $7.74-09$ & $5.52-09$ \\
\hline 4 & 14.2 & $6.33-09$ & $7.71-09$ & $5.41-09$ \\
\hline 5 & 13.8 & $6.19-09$ & $7.49-09$ & $5.48-09$ \\
\hline 6 & 12.8 & $6.00-09$ & 7. 21-09 & $5.44-09$ \\
\hline 7 & 12.2 & $5.93-09$ & $6.89-09$ & $5.30-09$ \\
\hline 8 & 11.1 & $5.68-09$ & $6.49-09$ & $5.09-09$ \\
\hline 9 & 10.0 & $5.53-09$ & $6.23-09$ & $4.83-09$ \\
\hline 10 & 9.0 & $5.22-09$ & $6.10-09$ & $4.85-09$ \\
\hline 11 & 8.2 & $5.12-09$ & $5.97-09$ & $4.84-09$ \\
\hline 12 & 7.4 & $4.86-09$ & $5.82-09$ & $4.55-09$ \\
\hline 13 & 6.4 & $4.56-09$ & $5.57-09$ & $4.46-09$ \\
\hline 14 & 5.0 & $4.32-09$ & $5.34-09$ & $4.26-09$ \\
\hline 15 & 4.7 & $4.25-09$ & $5.09-09$ & $4.33-09$ \\
\hline 16 & 4.1 & $4.04-09$ & $4.56-09$ & $4.06-09$ \\
\hline 17 & 3.0 & $3.43-09$ & $4.00-09$ & $3.37-09$ \\
\hline 18 & 2.4 & $3.14-09$ & $3.82-09$ & $3.14-09$ \\
\hline 19 & 2.3 & $3.07-09$ & $3.74-09$ & $3.09-09$ \\
\hline 20 & 1.8 & $2.65-09$ & $3.52-09$ & $2.66-09$ \\
\hline 21 & 1.1 & $2.01-09$ & $2.94-09$ & $2.01-09$ \\
\hline 22 & $5.5-01$ & $1.25-09$ & $1.69-09$ & $1.21-09$ \\
\hline 23 & $1.6-01$ & $7.68-10$ & $9.72-10$ & $7.53-10$ \\
\hline 24 & $1.1-01$ & $5.35-10$ & $7.45-10$ & $4.97-10$ \\
\hline 25 & $5.2-02$ & $3.02-10$ & $5.89-10$ & $2.53-10$ \\
\hline 26 & $2.5-02$ & $2.05-10$ & $5.13-10$ & $1.72-10$ \\
\hline 27 & $2.2-02$ & $1.41-10$ & $4.49-10$ & $1.25-10$ \\
\hline 28 & $1.0-02$ & $6.01-11$ & $4.21-10$ & 0.0 \\
\hline 29 & $3.4-03$ & $2.12-11$ & $4.79-10$ & 0.0 \\
\hline 30 & $1.2-03$ & $8.88-12$ & $5.25-10$ & 0.0 \\
\hline 31 & $5.8-04$ & $3.07-12$ & $5.58-10$ & 0.0 \\
\hline 32 & $1.0-04$ & 1. $14-12$ & $5.94-10$ & 0.0 \\
\hline 33 & $2.9-05$ & 1. $12-12$ & $6.18-10$ & 0.0 \\
\hline 34 & $1.1-05$ & $1.69-12$ & $6.28-10$ & 0.0 \\
\hline 35 & $3.1-06$ & $2.82-12$ & $6.22-10$ & 0.0 \\
\hline 36 & $1.1-06$ & $4.60-12$ & $6.00-10$ & 0.0 \\
\hline 37 & $4.1-07$ & $2.20-10$ & $5.17-10$ & 0.0 \\
\hline & $1.0-11$ & & & \\
\hline
\end{tabular}


4.B. Gamma Responses in $\operatorname{Rads} /\left(\right.$ Photon $/ \mathrm{cm}^{2}$ )

\begin{tabular}{|c|c|c|c|}
\hline Group & $\begin{array}{c}\text { Upper Energy } \\
\text { Boundary (MeV) } \\
\end{array}$ & $\begin{array}{c}\text { Gamma Frce-in-Air } \\
\text { Tissue Kerma } \\
\end{array}$ & $\begin{array}{l}\text { Claiborne-Trubey } \\
\text { Gamma Tissue Dose } \\
\end{array}$ \\
\hline 1 & 14.0 & $3.04-09$ & $3.05-09$ \\
\hline 2 & 10.0 & $2.41-09$ & $2.43-09$ \\
\hline 3 & 8.0 & $2.09-09$ & $2.12-09$ \\
\hline 4 & 7.0 & $1.88-09$ & $1.92-09$ \\
\hline 5 & 6.0 & $1.67-09$ & $1.71-09$ \\
\hline 6 & 5.0 & $1.46-09$ & $1.50-09$ \\
\hline 7 & 4.0 & $1.23-09$ & $1.27-09$ \\
\hline 8 & 3.0 & $1.05-09$ & $1.09-09$ \\
\hline 9 & 2.5 & $9.15-10$ & $9.59-10$ \\
\hline 10 & 2.0 & $7.68-10$ & $8.13-10$ \\
\hline 11 & 1.5 & $5.98-10$ & $6.41-10$ \\
\hline 12 & 1.00 & $4.36-10$ & $4.82-10$ \\
\hline 13 & 0.70 & $3.05-10$ & $3.60-10$ \\
\hline 14 & 0.45 & $1.98-10$ & $2.48-10$ \\
\hline 15 & 0.30 & $1.11-10$ & $1.64-10$ \\
\hline 16 & 0.15 & $5.56-11$ & $1.01-10$ \\
\hline 17 & 0.10 & $4.02-11$ & $7.44-11$ \\
\hline 18 & 0.07 & $4.09-11$ & $7.73-11$ \\
\hline 19 & 0.045 & $7.34-11$ & $1.17-10$ \\
\hline 20 & 0.030 & $1.63-10$ & $2.23-10$ \\
\hline \multirow[t]{2}{*}{21} & 0.020 & $5.22-10$ & $6.26-10$ \\
\hline & 0.010 & & \\
\hline
\end{tabular}


an acronym for Kinetic Energy Release in MAterials. The elemental kerma factors given here must be multiplied by the normal atomic number density (atoms $/ \mathrm{g} / 10^{24}$ ) to give calculational units of $\mathrm{rads} /\left(\right.$ neutron $/ \mathrm{cm}^{2}$ ), similar to those given in the tissue kerma responses 1 and 2 . The elemental kerma data included here were used in the generation of responses 1 and 2 . Response 36 is an older neutron tissue dose function, ${ }^{9}$ while responses 37 and 38 are older free-in-air tissue kerma factors, ${ }^{10}$ still frequently employed.

A word of caution should be expressed regarding the use of response 37 , since the Henderson neutron tissue kerma has no values below group 27 (10 keV), while the newer kerma have values at all energies and have a fairly significant value at thermal energies. Response 39 is the Snyder-Auxier neutron tissue dose response in units of rem/(neutron/ $\mathrm{cm}^{2}$ ). The data in response 6 , the Snyder-Auxier neutron tissue dose in rads/(neutron $/ \mathrm{cm}^{2}$ ), were multiplied by a quality factor which accounts for the difference in linear energy transfer (LET) for neutrons of different energies in order to obtain response 39. The LET quality factors are similar to the relative biological effectiveness (RBE) factors frequently used in radiobiological applications. Selected neutron and gamma sources are given in Table 5 in the 37-21 group structure. These sources are commonly used in defense-related calculations, and some of the neutron sources were utilized in the comparative calculations presented in Section IV. 
Table 5. SELECTED SOURCE DISTRIBUTIONS

5.A. Neutron Sources

Upper Energy

Group Boundary (MeV)

19.6

16.9

14.9

14.2

13.8

12.8

12.2

11.1

10.0

9.0

8.2

7.4

6.4

5.0

4.7

4.1

3.0

2.4

2.3

1.8

1.1

5. 5-01

1.6-01

1.1-01

5.2-02

2. 5-02

2. 2-02

1. 0-02

3. 4-03

1. 2-03

5. 8-04

1. 0-04

2.9-05

1.1-05

3.1-06

1.1-06

4.1-07

1. 0-11
$14 \mathrm{MeV}$

0.0

1. 89-02*

3.12-01

3.12-01

3.54-01

4. 37-03

0.0

0.0

0.0

0.0

0.0

0.0

0.0

0.0

0.0

0.0

0.0

0.0

0.0

0.0

0.0

0.0

0.0

0.0

0.0

0.0

0.0

0.0

0.0

0.0

0.0

0.0

0.0

0.0

0.0

0.0

0.0

1.00

Sources (Neutrons/Source Neutron)

Thermonuclear** Fission***

0.0

1.89-02

9.34-03

2.66-02

$1.67-02$

$1.69-02$

$1.24-02$

$7.48-03$

$6.82-03$

$6.78-03$

$1.03-02$

1.81-02

$3.62-03$

1. 24-02

2.60-02

$2.37-02$

$3.75-03$

$2.56-02$

6.44-02

8.85-02

9.14-02

1.16-02

1.11-01

5.40-02

5.68-03

9.26-02

1. 16-01

7. 38-02

2. 32-02

2.03-02

1.90-03

0.0

0.0

0.0

0.0

0.0

1.00
0.0

0.0

0.0

0.0

0.0

0.0

0.0

0.0

3. 84-03

3. 50-03

5. 39-03

7. 35-03

1.84-02

3. 25-03

8.47-03

5. 50-02

3. 24-02

1.06-02

9.72-02

1.47-01

2.16-01

1. 50-01

1.93-02

1.21-01

5.73-02

6.00-03

2.40-02

1.44-02

0.0

0.0

0.0

0.0

0.0

0.0

0.0

0.0

0.0

1.00

*Read 1.89-02 as $1.89 \times 10^{-2}$.

**From reference 11 .

***From reference 12 . 
5.B. Prompt Gamma Fission Source*

\begin{tabular}{|c|c|c|}
\hline Group & $\begin{array}{l}\text { Upper Energy } \\
\text { Boundary (MeV) }\end{array}$ & Gamma/Source Gamma \\
\hline 1 & 14.0 & $1.65-5^{* *}$ \\
\hline 2 & 10.0 & $1.34-4$ \\
\hline 3 & 8.0 & $3.02-4$ \\
\hline 4 & 7.0 & $9.08-4$ \\
\hline 5 & 6.0 & $2.73-3$ \\
\hline 6 & 5.0 & $8.19-3$ \\
\hline 7 & 4.0 & $2.46-2$ \\
\hline 8 & 3.0 & $2.70-2$ \\
\hline 9 & 2.5 & $4.69-2$ \\
\hline 10 & 2.0 & $8.12-2$ \\
\hline 11 & 1.5 & $1.41-1$ \\
\hline 12 & 1.00 & $1.30-1$ \\
\hline 13 & 0.70 & $1.47-1$ \\
\hline 14 & 0.45 & $1.09-1$ \\
\hline 15 & 0.30 & $1.29-1$ \\
\hline 16 & 0.15 & $4.79-2$ \\
\hline 17 & 0.10 & $3.01-2$ \\
\hline 18 & 0.070 & $2.58-2$ \\
\hline 19 & 0.045 & $1.58-2$ \\
\hline 20 & 0.030 & $1.07-2$ \\
\hline \multirow[t]{2}{*}{21} & 0.020 & $1.08-2$ \\
\hline & 0.010 & 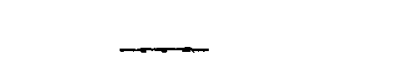 \\
\hline TOTAL & & 0.98 \\
\hline
\end{tabular}

*From reference 13. $* *$ Read as $1.65 \times 10^{-5}$. 


\section{TESTING}

This section presents the results of efforts designed to determine the ability of the 37-21 library to solve problems of basic interest to the defense community. These efforts were specifically directed toward questions concerning the correctness of the basic data, the results of the processing (including effects of the weighting function utilized in collapsing from the basic point data to a few-group library), and the adequacy of the 37 neutron and 21 gamma energy group structures selected. Three basic problems were addressed in the course of this study, two air penetration calculations and a concrete penetration calculation. A11 three problems were run using the familiar sources and response functions given in Section III and Appendix B.

Table 6 presents the results of an air transport calculation in spherical geometry using an $\mathrm{S}_{16}$ angular quadrature. Neutron and gamma tissue doses, neutron displacement in silicon, and neutron and gamma ionization in silicon are given at $2000 \mathrm{~m}$ in air due to a thermonuclear source. The calculations were extended to $3000 \mathrm{~m}$ to allow for adequate reflection. The results are given in rads or equivalent $1-\mathrm{MeV}$ displacements per incident neutron. This problem was calculated with six different cross-section sets. The 129-neutron 43-gamma cross-section set ${ }^{*}$ with a (1/E) weighting was selected as a standard because energy-group boundaries for this structure were specifically chosen to fit in detail the peaks and valleys in the neutron cross sections and to bracket major gammaproduction lines for elements involved in transport through air and concrete. The $129-43$ case with $\left(1 / E \Sigma_{T}\right)$ weighting indicates the adequacy of this group structure for this problem, since the results agree to within

* See Appendix D. 
ORNL-DWG . 74-2532R

Table 6. AIR TRANSPORT PROBLEM (200OM, THERMO SOURCE, $\mathrm{P}_{3} \mathrm{~S}_{16}$, SPHERICAL)

\begin{tabular}{|c|c|c|c|c|c|}
\hline Cross Section & $\begin{array}{c}\text { Snyder-Neufeld } \\
\text { Neutron TIssue } \\
\text { Dose (Rads) }\end{array}$ & $\begin{array}{l}\text { Neutron DIsplacement } \\
\text { (equiv. I-MeV/neutrons } / \mathrm{cm}^{2} \text { ) }\end{array}$ & $\begin{array}{c}\text { Neutron } \\
\text { Ionization } \\
\text { (Rads-S1) } \\
\end{array}$ & $\begin{array}{l}\text { Henderson Camma } \\
\text { Tissue Dose (Rads) }\end{array}$ & $\begin{array}{c}\text { Canma } \\
\text { Ionization } \\
\text { (Rads-S1) } \\
\end{array}$ \\
\hline $129-43(1 / E)$ & $1.61-23$ & $3.87-15$ & $2.72-25$ & $8.28-24$ & $7.81-24$ \\
\hline $129-43\left(1 / E \Sigma_{T}\right)$ & $+48 *$ & $+3 \%$ & $+1 \%$ & $+0 \%$ & $+2 \%$ \\
\hline $129-42(1 / E)$ & $-\cdots$ & -- & -- & $-15 \%$ & $-15 \%$ \\
\hline $37-18(1 / E)$ & $+3 \%$ & $+2 \%$ & $+1 \%$ & $+3 \%$ & $+3 \%$ \\
\hline $37-18\left(1 / E \Sigma_{T T}\right)$ & $+19 \%$ & $+14 \%$ & $+7 \%$ & $+4 \%$ & $+5 \%$ \\
\hline $37-21(1 / E)$ & $+3 \%$ & $+2 \%$ & $+1 \%$ & $+0 \%$ & $+18 \%$ \\
\hline
\end{tabular}

* Percent deviation from $129-43(1 / \mathrm{E})$ results. 
4\%. The 129-42 set indicates the importance ( $15 \%)$ of including the gammas with energies above $10 \mathrm{MeV}$, since the 42-group gamma structure cuts off at $10 \mathrm{MeV}$. The $37-18$ (1/E) results agree quite well with the 129-43 set and indicate the adequacy of the 37-group, neutron structure for this problem. The 37-18 $\left(1 / \mathrm{E} \Sigma_{\mathrm{T}}\right)$ results, on the other hand, show a fairly large discrepancy, exceeding the arbitrarily selected goal of agreement within $10 \%$. This is believed to be due to an overemphasis on relative minima contained in relatively broad energy groups in the 1.8 - to $0.1-\mathrm{MeV}$ range, so that resulting downscatter is too low for the $\left(1 / E \Sigma_{T}\right)$-weighted case. The 37-21 (1/E) results agree very well for the neutron responses and for the gamma tissue dose. The increase in the gamma silicon response is due to the importance of low-energy gammas $(\mathrm{E}<0.10 \mathrm{MeV})$ for the silicon ionization response as compared with the Henderson gamma tissue dose response (see Appendix B). The 21-group gamma structure was designed to provide sufficient energy groups for low-energy gamma transport (5 groups below $0.1 \mathrm{MeV}$ ) and hence gives an enhanced silicon ionization compared to the 43-group structure.

Table 7 presents the results of a series of calculations for a thermonuclear source spectrum incident on a $40 \mathrm{in}$. concrete slab. The responses and cross section sets utilized are very similar to those presented in Table 6 , but an $\mathrm{S}_{8}$ angular quadrature set was employed for the slab geometry. The results here are generally similar to those obtained for the air case. In general, the results were satisfactory (within $10 \%$ of the $129-43$ (1/E) results) for all combinations of weighting functions and group structures employed. The final calculation was 
Table 7. CONCRETE TRANSPORT PROBLEM ( $40^{\prime \prime}$ CONCRETE, THERMO SOURCE, $\mathrm{P}_{3} \mathrm{~S}_{8}, \mathrm{~S}$ lab)

\begin{tabular}{|c|c|c|c|c|c|}
\hline Cross Section & $\begin{array}{l}\text { Snyder-Neufeld } \\
\text { Neutron Tissue } \\
\text { Dose (Reds) } \\
\end{array}$ & $\begin{array}{l}\text { Neutron Displacement } \\
\text { (equiv. I-MeV/neutrons } / \mathrm{cm}^{2} \text { ) }\end{array}$ & $\begin{array}{c}\text { Neutron } \\
\text { Ionization } \\
\text { (Rads-Si) } \\
\end{array}$ & $\begin{array}{l}\text { Henderson Gamma } \\
\text { TIssue Dose (Rads) }\end{array}$ & $\begin{array}{c}\text { Gamma } \\
\text { Ionization } \\
\text { (Rads-Si) }\end{array}$ \\
\hline $129-43(1 / E)$ & $2.26-14^{*}$ & $4.97-6$ & $4.69-16$ & $4.72-14$ & $4.66-14$ \\
\hline $129-43\left(1 / \mathrm{E \Sigma}_{\mathrm{T}}\right)$ & $+7 \%$ & $-1 \%$ & $+3 \%$ & $+1 \%$ & $+1 \%$ \\
\hline $129-42(1 / E)$ & --- & -- & -- & $\%$ & $0 \%$ \\
\hline $37-18(1 / E)$ & $-7 \%$ & $-7 \%$ & $-3 \%$ & $-5 \%$ & $-4 \%$ \\
\hline $37-18\left(1 / \mathrm{E} \Sigma_{\mathrm{T}}\right)$ & $+2 \%$ & $+1 \%$ & $+1 \%$ & $-3 \%$ & $-1 \%$ \\
\hline $37-21(I / E)$ & $-7 \%$ & $-7 \%$ & $-4 \%$ & $-5 \%$ & $+2 \%$ \\
\hline $37-21(1 / E) * *$ & $+4 \%$ & $+4 \%$ & $+3 \%$ & $-3 \%$ & $+4 \%$ \\
\hline
\end{tabular}

*Percent deviation from $129-43(1 / E)$ results.

**ENDF/B-IV Si (4151-3) and Fe (4180-2). 
added to determine the effect of the new ENDF/B-IV evaluated data and does indicate a significant increase in calculated responses, especially due to the high-energy cross sections for silicon.

Table 8 presents the results of a study of very deep (9000 m) air penetration from $14-\mathrm{MeV}$ and weapon fission sources. Results for the $14 \mathrm{MeV}$ case indicate that both weightings meet the $10 \%$ criteria for about $4000 \mathrm{~m}$, and that the $37-21$ results are always within a $20 \%$ criteria for this problem. These results are quite gratifying, since the uncertainty in the dose due to uncertainties in the basic cross-section data is considerably larger than this discrepancy. ${ }^{2}$ The weapon fissionweighted cross section set displays a definite superiority to the $1 / \mathrm{E}-$ weighted set for the weapon fission source within the first $3000 \mathrm{~m}$. The weapon fission-weighted cross sections for nitrogen and oxygen were therefore included in this library for use in similar problems.

A related study to determine $\mathrm{P}_{\ell}$ (degree of Legendre expansion) requirements for air transport determined that the $\mathrm{P}_{3}$ expansion was adequate. For example, with a weapon fission source, the resulting Snyder-Auxier neutron dose from a $\mathrm{P}_{3}$ calculation was within $7 \%$ of a similar $\mathrm{P}_{5}$ calculation out to $9000 \mathrm{~m}$, while the gamma dose was within $13 \%$.

In summary, these results indicate that the 37-21 library is adequate to solve many problems of interest to the defense community. Additional testing of the library continues, and additions and updates will be performed as required. 
Table 8. NEUTRON TISSUE DOSE ${ }^{a}$ COMPARISONS

FOR $14 \mathrm{MeV}$ AND WEAPON FISSION SOURCES IN $9000 \mathrm{~m}$ OF AIR

\begin{tabular}{|c|c|c|c|c|c|c|}
\hline \multirow[b]{2}{*}{$\begin{array}{c}\text { Cross Sections Weighting } \\
\text { Range (m) } \\
\end{array}$} & & \\
\hline & $\begin{array}{c}129-43 \\
1 / E \\
\end{array}$ & $\begin{array}{c}37-21 \\
1 / E \\
\end{array}$ & $\begin{array}{c}37-21 \\
\text { Weapon Fission } \\
\phi \text { Weighted } \\
\end{array}$ & $\begin{array}{c}129-43 \\
1 / \mathrm{E} \\
\end{array}$ & $\begin{array}{l}37-21 \\
1 / E \\
\end{array}$ & $\begin{array}{c}37-21 \\
\text { Weapon Fission } \\
\phi \text { Weighted } \\
\end{array}$ \\
\hline 500 & $1.72-19^{b}$ & $+0 \%^{c}$ & $-0 \%$ & $1.05-19$ & $+5 \%$ & $+4 \%$ \\
\hline 1000 & $1.25-20$ & $+0 \%$ & $-2 \%$ & $3.93-21$ & $+12 \%$ & $+5 \%$ \\
\hline 1500 & $9.75-22$ & $+1 \%$ & $-2 \%$ & $1.65-22$ & $+19 \%$ & $+8 \%$ \\
\hline 2000 & $8.03-23$ & $+0 \%$ & $-3 \%$ & $8.58-24$ & $+18 \%$ & $+7 \%$ \\
\hline 2500 & $7 \cdot 31-24$ & $-0 \%$ & $-4 \%$ & $5.82-25$ & $+12 \%$ & $+2 \%$ \\
\hline 3000 & $6.64-25$ & $-2 \%$ & $-6 \%$ & $4.41-26$ & $+4 \%$ & $-5 \%$ \\
\hline 3500 & $6.01-26$ & $-5 \%$ & $-8 \%$ & $3.58-27$ & $-4 \%$ & $-10 \%$ \\
\hline 4000 & $5.50-27$ & $-7 \%$ & $-11 \%$ & $3.07-28$ & $-9 \%$ & $-14 \%$ \\
\hline 4500 & $5.88-28$ & $-9 \%$ & $-12 \%$ & $3.14-29$ & $-12 \%$ & $-15 \%$ \\
\hline 5000 & $5.54-29$ & $-11 \%$ & $-14 \%$ & $2.86-30$ & $-14 \%$ & $-17 \%$ \\
\hline 5500 & $6.05-30$ & $-12 \%$ & $-16 \%$ & $3.03-31$ & $-14 \%$ & $-17 \%$ \\
\hline 6000 & $5.78-31$ & $-13 \%$ & $-16 \%$ & $2.82-32$ & $-14 \%$ & $-17 \%$ \\
\hline 6500 & $5.54-32$ & $-14 \%$ & $-17 \%$ & $2.63-33$ & $-12 \%$ & $-15 \%$ \\
\hline 7000 & $5 \cdot 31-33$ & $-13 \%$ & $-16 \%$ & $2.46-34$ & $-10 \%$ & $-13 \%$ \\
\hline 7500 & $5.85-34$ & $-13 \%$ & $-16 \%$ & $2.64-35$ & $-6 \%$ & $-10 \%$ \\
\hline 8000 & $5.61-35$ & $-11 \%$ & $-15 \%$ & $2.47-36$ & $-2 \%$ & $-7 \%$ \\
\hline 8500 & $5 \cdot 36-36$ & $-9 \%$ & $-13 \%$ & $2 \cdot 30-37$ & $+4 \%$ & $-2 \%$ \\
\hline 9000 & $3.17-37$ & $-6 \%$ & $-11 \%$ & $1.32-38$ & $+10 \%$ & $+4 \%$ \\
\hline
\end{tabular}

a. Snyder-Neufeld neutron response function, see Appendix B, in Rads/Incident Neutron.

b. Read as $1.72 \times 10^{-19}$

c. Percent difference from the value calculated with the $129-43$ cross section set. 


\section{REFERENCES}

1. N. M. Greene, et al, "AMPX: A llodular Code System for Generating Coupled Multigroup Neutron-Gamma Libraries from ENDF/B," ORNL-TM3706 (March 1976).

2. D. Garber, C. Dunford, and S. Pearlstein, "Data Formats and Procedures for the Evaluated Nuclear Data File, ENDF," BNL-NCS-50496 (ENDF 102) Revised Oct. 1975.

3. D. E. Bartine, E. M. Oblow, F. R. Mynatt, "Radiation-Transport Cross-Section Sensitivity Analysis - A General Approach Illustrated for a Thermonuclear Source in Air," Nuc. Sci. Eng. 55, 147 (1974).

4. W. W. Engle, Jr., "A User's Manual for ANISN, A One-Dimensional Discrete Ordinates Transport Code with Anisotropic Scattering," K-1693, Computing Technology Center (1967).

5. J. J. Ritts, M. Solomito, P. N. Stevens, "The Calculation of Neutron-Induced Physical Doses in Human Tissues," ORNL-TM-2991 (1969).

6. M. A. Abdou, C. W. Maynard, R. Q. Wright, "MACK: A Computer Program to Calculate Neutron Energy Release Parameters (Fluenceto-Kerma Factors) and Multigroup Neutron Reaction Cross Sections From Nuclear Data in ENDF Format," ORNL-TM-3994 (July 1973).

7. "Protection Against Neutron Radiation," NCRP-38, National Council on Radiation Protection and Measurements, Washington, D. C. (1971).

8. H. C. Claiborne, D. K. Trubey, "Dose Rates in a Slab Phantom from Monoenergetic Gamma Rays," Nucl. Appl. Tech. 8, 450 (1970).

9. W. S. Snyder and C. Neufeld, Radiation Res., 6, 1, 67 (1957).

10. B. J. Henderson, "Conversion of Neutron or Gamma Ray Flux to Absorbed Dose Rate," XDC 59-8-179 (August 14, 1959).

11. E. A. Straker and M. L. Gritzner, "Neutron and Secondary Gamma-Ray Transport in Infinite Homogeneous Air," ORNL-TM-4464 (1969).

12. J. V. Pace, III, D. E. Bartine, F. R. Mynatt, "Neutron and Secondary-Gamma-Ray Transport Calculations for $14-\mathrm{MeV}$ and Fission Neutron Sources in Air-Over-Ground and Air-Over-Seawater Geometries," ORNL-TM-4841 (August 1975).

13. P. G. Fisher, W. S. Knapp, "Aids for the Study of Electromagnetic Blackout," 70TMP-12, General Electric - TEMP, Santa Barbara, Cal., p. 2.7 (July 1970). 
- 
APPENDIX A

Listing of Partial Cross Sections

This appendix contains partial cross sections listed by nuclide for each cross-section set included in the library. Partials are included for both neutron and gamma cross sections in a one-dimensional array printed by the AMPX code at the completion of processing the point data into multigroup form. The total cross section for neutrons is the sum of the elastic, inelastic, $(n, 2 n)$, and absorption cross sections. The constituent partials of the absorption cross section $[\operatorname{such}$ as $(n, \gamma),(n, P),(n, D),(n, T),(n, \alpha)$, etc.] are then listed separately. The inelastic cross section includes $\left(n, n^{\prime}, x \gamma\right)$-type reactions. The gamma partials listed are the pair-production and photoelectric cross sections and their sum, printed as the absorption cross section. The Compton scattering cross section is not given here, but may be obtained by subtracting the absorption cross section from the total nuclide cross section. The energy deposition values given here are gamma kerma values in units of (MeV-barn)/(photon-atom), and are consistent with the tissue kerma values in ( $\mathrm{rads} /$ atom)/(photon/ $\mathrm{cm}^{2} / \mathrm{gram}$ ) given in Appendix B. No gamma partials are listed for the weapon fission-weighted nitrogen and oxygen cross-section sets, since the gamma transport cross sections are unaffected by the weighting function and are therefore the same as those listed under the $(1 / E)$-weighted nitrogen and oxygen. The partial cross sections listed can be used for analytical purposes or as response functions to determine reaction rates. 
ORNL-DWG $76-10087_{\mathrm{R}}$

REACTION CROSS SECTICNS FISR HYDROGEN ONA $4148 / 2$ (1/E WT. I

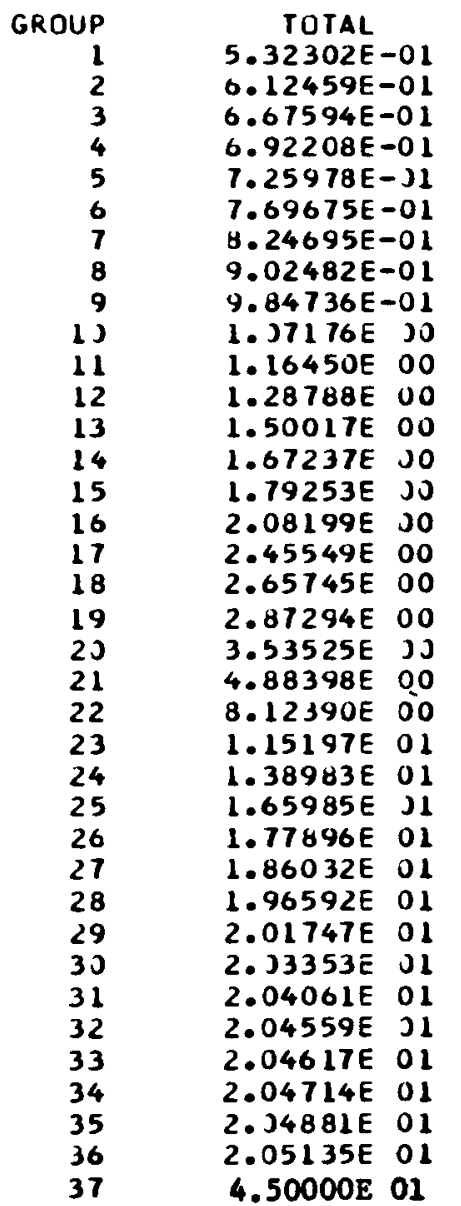

\begin{tabular}{|c|c|}
\hline $\begin{array}{c}\text { ELASTIC } \\
5.32275 E-01 \\
6.12431 E-01 \\
6.67565 E-01 \\
6.92178 E-C 1 \\
7.25548 E-J 1 \\
1.65645 E-01 \\
8.24664 E-01 \\
9 . C 2450 E-01 \\
5.84703 E-C 1 \\
1.07172 E \text { DO } \\
1.16447 E \text { CC } \\
1.28785 E \text { OO } \\
1.50014 E 0 C \\
1.67234 E \text { CO } \\
1.75250 E 00 \\
2.68195 E \text { CO } \\
2.45546 E 00 \\
2.65741 E 00\end{array}$ & $\begin{array}{l}\text { AB SORPTION } \\
2.68673 E-05 \\
2.83339 E-05 \\
2.93463 E-05 \\
2.97573 E-05 \\
3.30655 E-05 \\
3.06026 E-05 \\
3.13505 E-05 \\
3.21841 E-05 \\
3.29407 E-05 \\
3.35589 E-05 \\
3.42191 E-05 \\
3.52371 E-05 \\
3.55527 E-05 \\
3.63973 E-05 \\
3.63248 E-05 \\
3.55786 E-05 \\
3.52368 E-05 \\
3.47338 E-05\end{array}$ \\
\hline 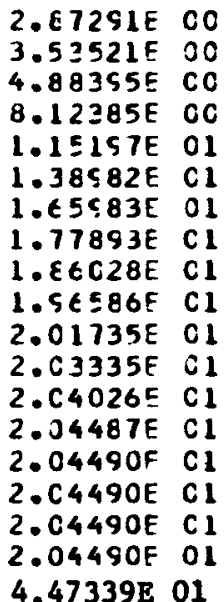 & $\begin{array}{l}3.43736 E-05 \\
3.43693 E-05 \\
3.53676 E-05 \\
5.13731 E-05 \\
8.71564 E-05 \\
1.32163 E-04 \\
2.21885 E-04 \\
2.93816 E-04 \\
3.89943 E-04 \\
6.82155 E-04 \\
1.18550 E-03 \\
1.81401 E-03 \\
3.48742 E-03 \\
7.28390 E-03 \\
1.271 C 7 E-02 \\
2.24407 E-02 \\
3.91590 E-02 \\
6.45676 E-02 \\
2.66028 E-01\end{array}$ \\
\hline
\end{tabular}

PHOTCA INTERACTION CRCSS SECTICAS

$\begin{array}{cc}\text { GROUP } & \text { SIG ABS } \\ 1 & 3.8 C 646 E-03 \\ 2 & 2.91026 E-03 \\ 3 & 2.32310 E-33 \\ 4 & 1.95515 E-03 \\ 5 & 1.60361 E-03 \\ 6 & 1.20935 E-C 3 \\ 7 & 7.62591 E-04 \\ 8 & 4.36222 E-04 \\ 9 & 2.50127 E-04 \\ 10 & 9.89497 E-05 \\ 11 & 1.18670 E-C 5 \\ 12 & 1.97341 E-09 \\ 13 & 6.76240 E-09 \\ 14 & 2.53930 E-08 \\ 15 & 1.56099 E-C 7 \\ 16 & 8.97628 E-07 \\ 17 & 3.17157 E-06 \\ 18 & 1.23380 E-05 \\ 19 & 5.18057 E-05 \\ 20 & 2.37748 E-04 \\ 21 & 1.46685 E-03\end{array}$

SIG PE

$3.75934 E-11$ $5.10629 E-11$ $6.27394 E-11$ $7.39797 \mathrm{E}-11$ 8.96976E-11 $1.14444 \mathrm{E}-10$ $1.57350 E-10$ $2.14886 E-10$ $2.86445 E-10$

$4.32054 E-10$

$7.92342 \mathrm{E}-10$ $1.97341 E-09$ $6.76240 \mathrm{E}-09$ $2.53930 E-08$ $1.56099 \mathrm{E}-07$ $8.97628 E-07$ $3.17157 \mathrm{E}-06$ 1.23380E-05 $5.18057 E-05$ $2.07748 E-04$ $1.46685 E-03$
SIG PP

$3.8 J 646 E-J 3$
$2.910 \angle 6 E-J 3$
$2.32 J 10 E-J 3$
$1.9 J 515 E-J 3$
$1.6 J 161 E-J 3$
$1.2 J 935 E-J 3$
$7.52591 E-J 4$
$4.36222 E-J 4$
$2.50127 E-J 4$
$9.844 Y 2 E-J 5$
$1.18662 E-J 5$
0.0
0.0
0.0
0.0
0.0
0.0
0.0
0.0
0.0
0.0

ENERGY DEP

4. 13745E-O1 3. $57840 E-01$ 3. 26230 E- 01 3.03612E-01 2.79006E-01 2. $51195 E-01$ 2. 18681 E- 01 1. $90105 E-01$ $1.67767 \mathrm{EO}$ $1.41816 E-01$ 1. $10723 E-01$ 8.08160E-02 5. $64788 \mathrm{E}-02$ 3.65513E- 02 $2.03433 \mathrm{E}-02$ $9.40621 \mathrm{E}-03$ 5. 33661 E- 03 2.88521 E- 03 1.41804 E- 03 7. 47593 E- 04 4.99627 E- 04 

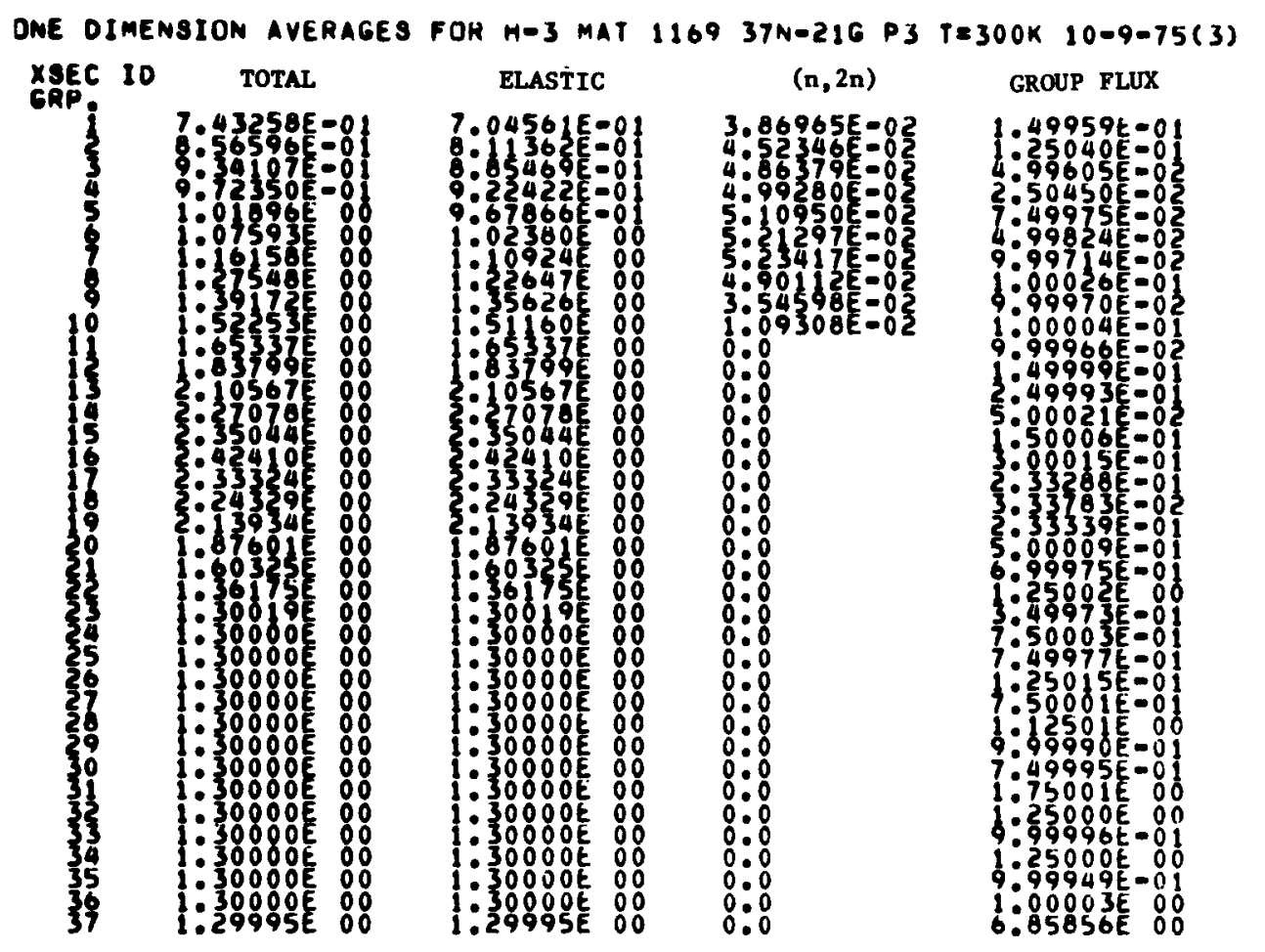

IDENTIFIEK

1169

PMOTON INTERACTION CROSS SECTIONS

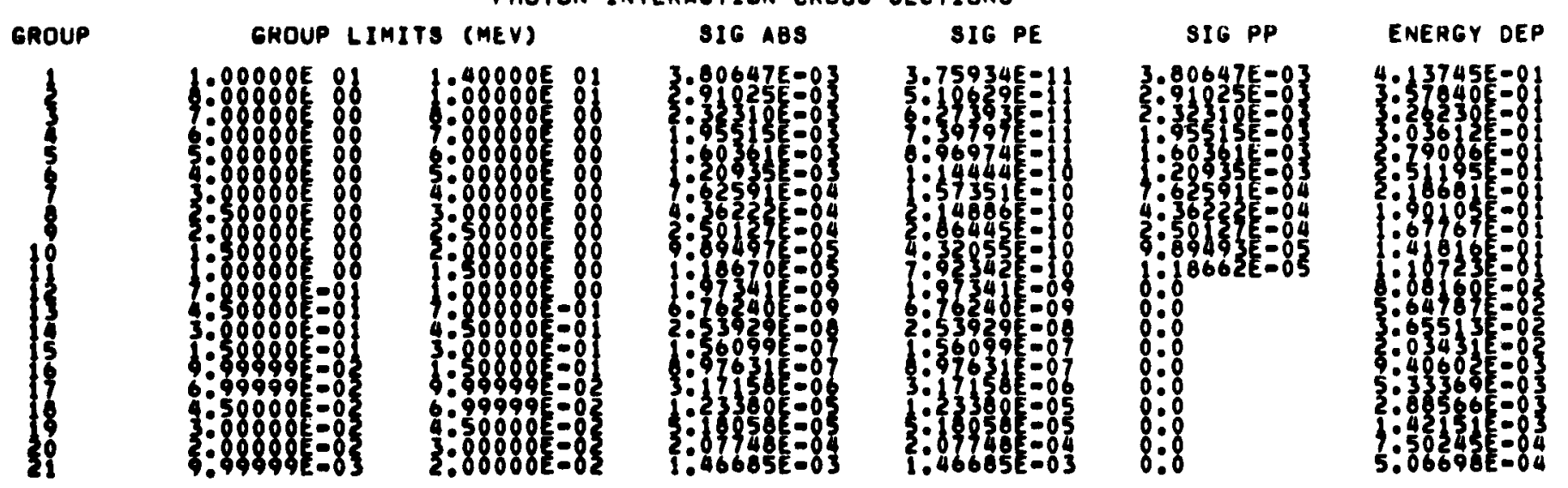




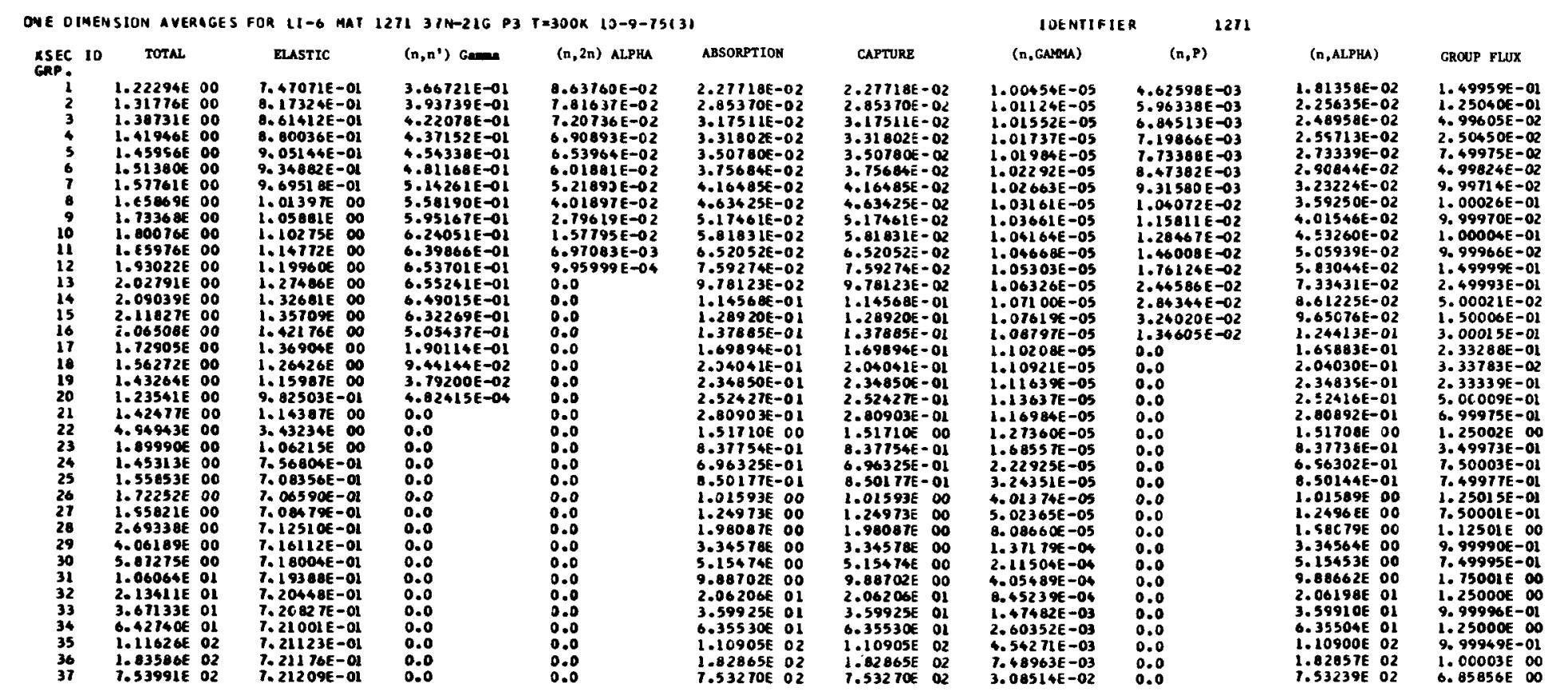


PHOTON INTERACTION CROSS SECTIONS

GROUP
1
2
3
4
5
6
7
8
9
10
11
12
13
14
15
16
17
18
19
20
21

GROUP LIMITS (MEV)

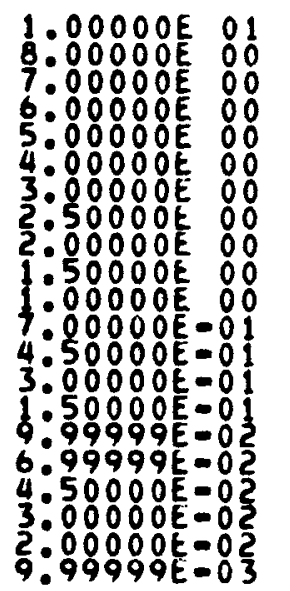

SIG ABS

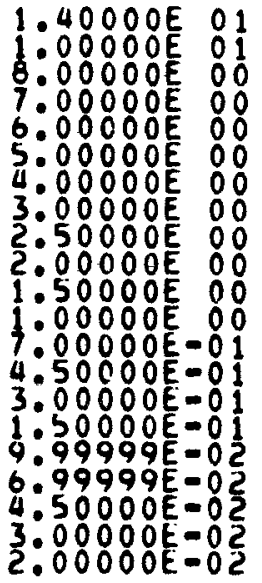

2. $57996 E-02$ 2.03699E $=0$ ? $1.44115 E=02$ $1: 210017 E=02$ $6: 27093=03$ $3: 77246 E=03$ $2.22000 E-03$ $8.91318 E-04$ 7. $12826 E=05$ $7.20421 E=07$ $6.85720=06$ $3.86017 E-05$ 2. $18320 E=04$ $7.82383 E=04$ $3 \cdot \begin{aligned} & 5577 E=03 \\ & 1: 3123 E=02\end{aligned}$ $5: 472255 \mathrm{E}=01$
SIG PE

$1.30699 E-08$ $2.04830 E=08$ 2: 96879 E $-O B$ 3.60036E-08 $4.60196 E-08$ $6: 35137 E=08$ $0.60000 \mathrm{E}=0$ $1.16165 E=07$ $1.75301 E-07$ 3.ट $1113 E-07$ $7.20421 E=07$ $2.06655 E-06$ $6.85720 E=06$ $3.86017 E-05$ 2. $18320 E=04$ $7.82383 E-04$ 1. $39123 \mathrm{E}=02$ $5.47014 E=02$
$4.42225 E=01$
SIG P

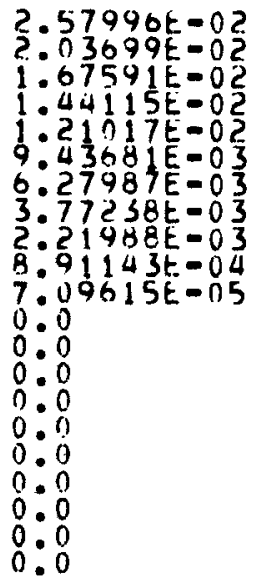

\section{ENERGY UEP}

$1.40012 t \quad 0$ ) $1.16681 \mathrm{E} 01$ 9. $57772 \mathrm{ZE}=0$ ? $8.69787 E-0$ S.76 72 CE-0 $5.05144 \bar{E}=0$ $4.25015=0$ 3. 321 द $3=0$ $2.424405=0$ $1.69437 E=0$ $1.09656=0$ $6: 10369 E=0$ $2.82438 E-02$ $1.60644 E-02$ 8.82651E=03 3:6 34 ? $1 E=03$ 


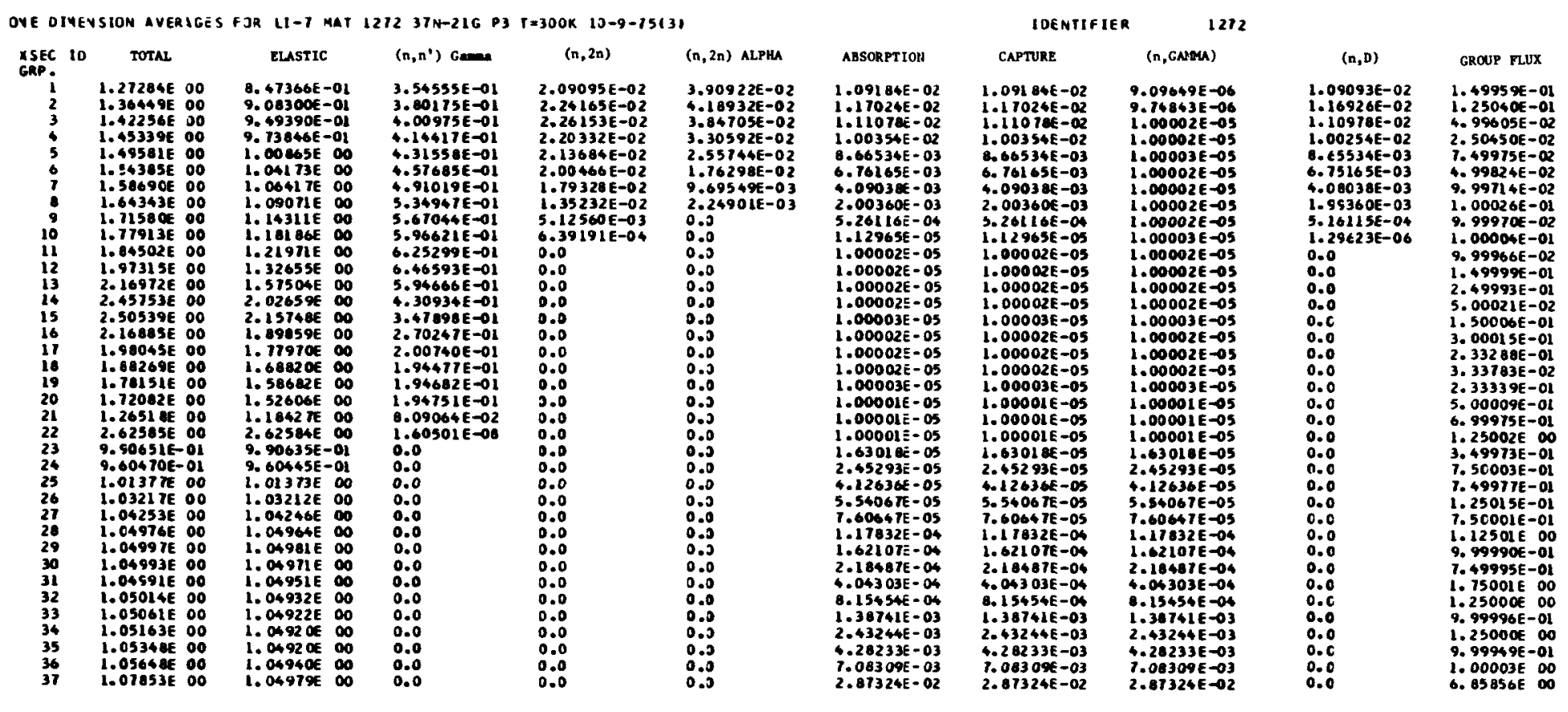


REATI ION CROSS SECTIONS FOR BE $4154 / 3$ 37N-21G 1/E 11-20-74 (5

JOENTIFICAIION

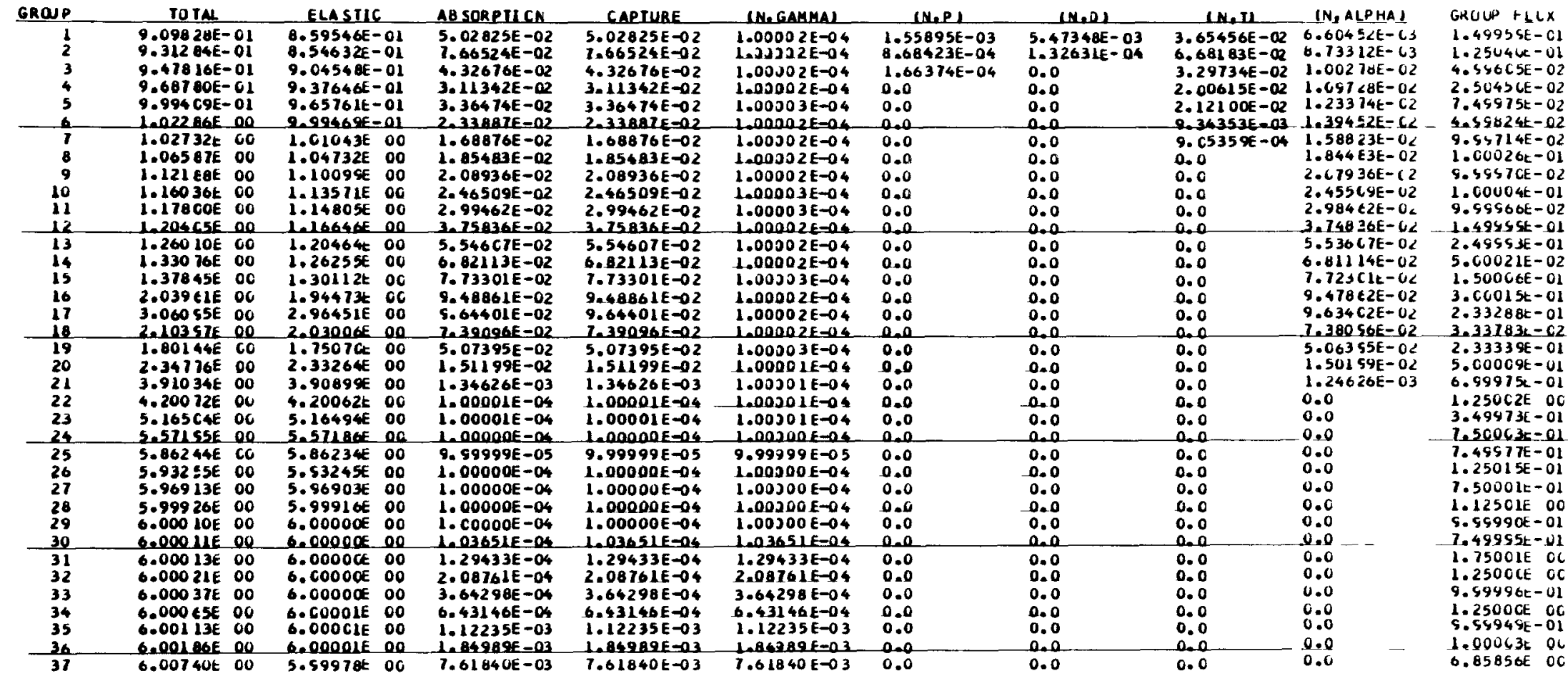


PHOTON INTERACTION CROSS SECTIONS FOR Be

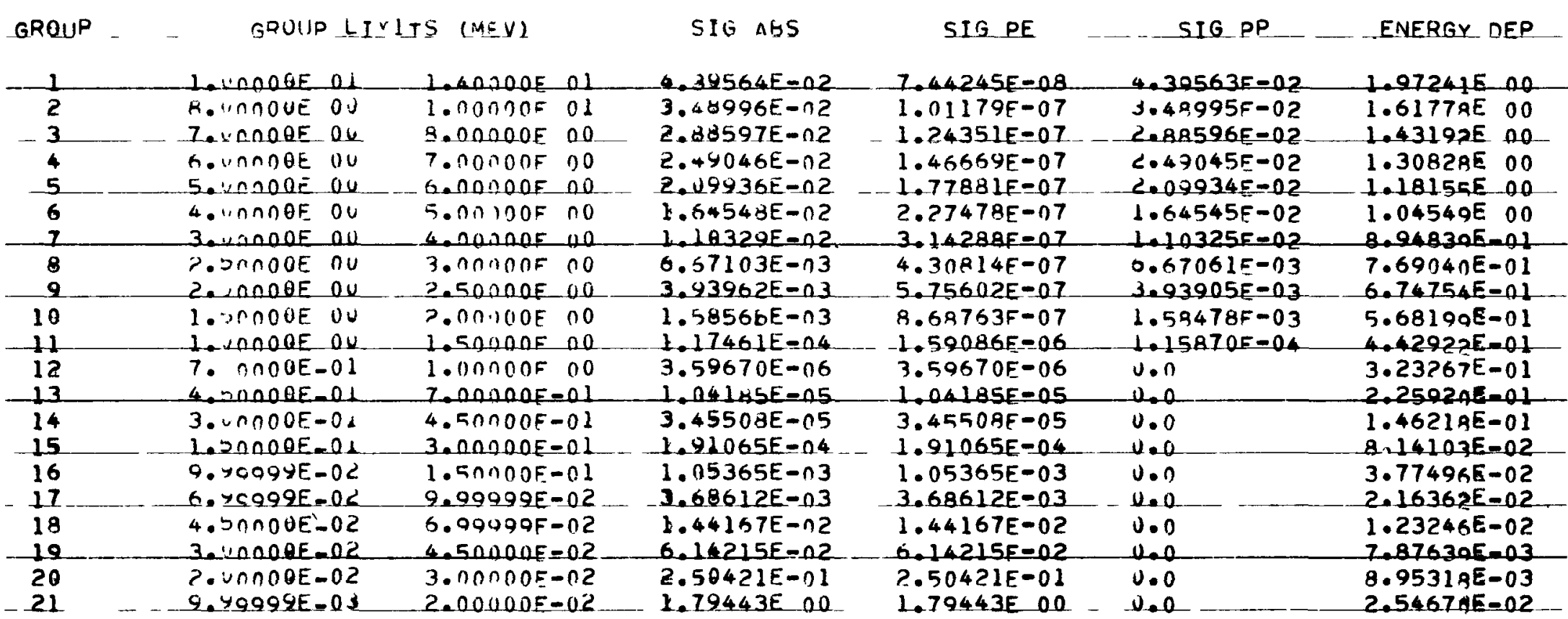




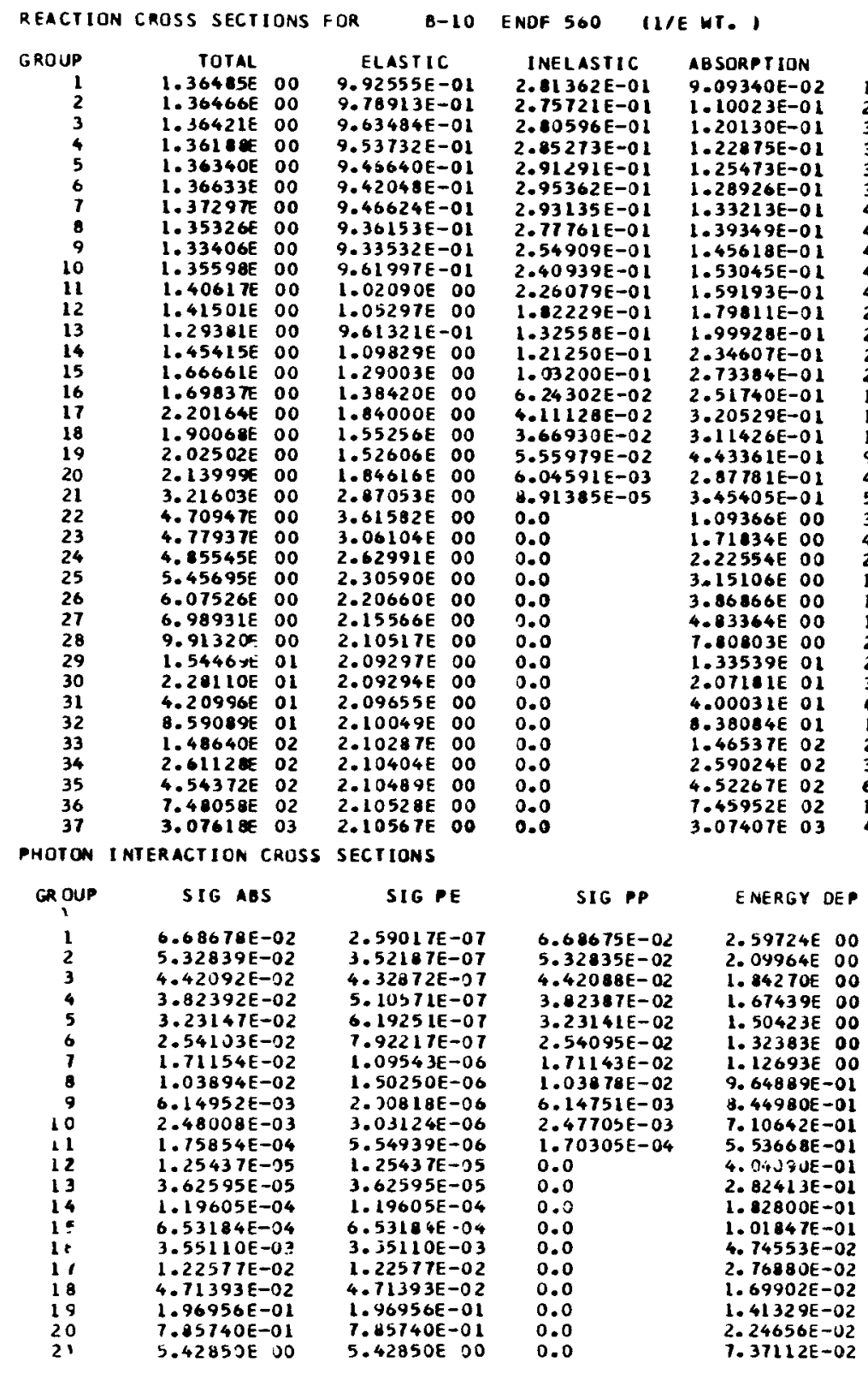

IOENTIFICATION

$(N, P)$

$(\mathrm{N}, \mathrm{O})$

(N,ALPHA) GROUP FLUX

5. $01917 E-02 \quad 1.49958 E-01$

6.0243LE-02 4.99605E-02

6.01776E-02 2.50451E-02

$5.92245 E-02 \quad 1.49978 E-02$

$5.84742 E-02 \quad 4.99823 E-02$

$5.02425 E-02$ $9.9975 E-02$

$6.13103 E-02 \quad 9.99977 E-02$

$6.73643 E-02 \quad 1.00005 E-01$

Q.10065E-02 9.99966E-02

$1.17208 \mathrm{E}-01$ 1.49999E-0

$1.58342 E-01 \quad 2.49995 E-01$

$2.51299 E-01 \quad 1.50009 E-02$

$2.37402 E-01 \quad 3.00014 E-01$

$3.08371 E-01 \quad 2.33288 E-0$

3. $00538 E-01 \quad 3.33783 E-02$

2.33697E-OL $2.33341 E-0$

$3.44879 E-01$ 6. $99975 E-0$

$1.09362 E$ DO $1.25002 E 00$

$1.71933 E$ OO $3.49973 E-0$

$2.22554 E$ OO $7.50003 E-01$

$3.15106 E$ DO $7.49976 E-0$ I

$3.86866 E$ OO $1.25015 E-0$

4.83364E OO 7.5O000E-OI

..33539E OI 9 .

$2.0718 \mathrm{bE}$ OI $7.49995 \mathrm{E}-\mathrm{O}$

4.00031E O1 1.75001E 00

8.38084E OL 1.25000 OO

$1.46537 E$ O2 $9.99996 E-01$

$4.52267 E$ O2 $9.99949 E-0$

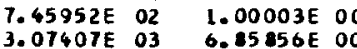


ONE OIMENSION AVERAGES FOR Q-11 MAT $110037 N-216$ P3 TE300K 10-9-73(3)
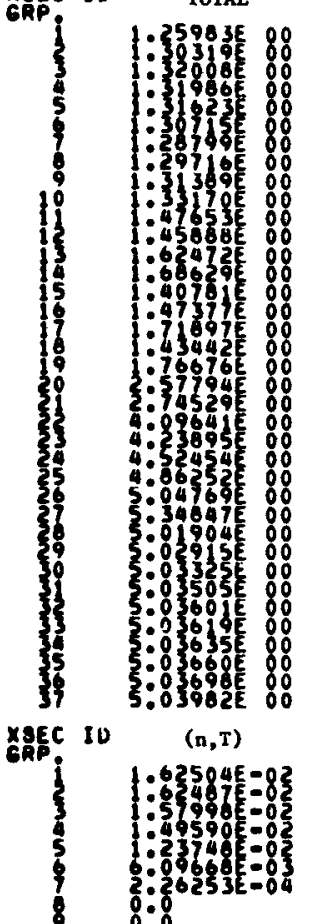

10

if

I.

II

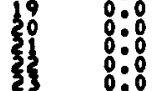

悲

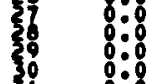

II $\quad 0: 0$

$\begin{array}{cc}3 & 8 \\ 35 & 8 \\ 3 & 8 \\ 0 & 0 \\ 0 & 0 \\ 0 & 0\end{array}$
ELASTIC

$\left(n, n^{\prime}\right)$ GArOA

$(n, 2 n)$

ABSORPTION

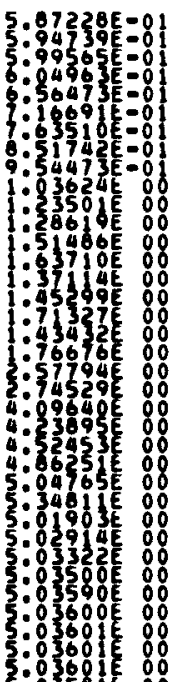

$.03581 E$ o

(n,ALPHA)

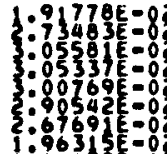

6:9031 $15=0$

$8: 8$

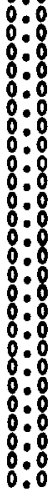

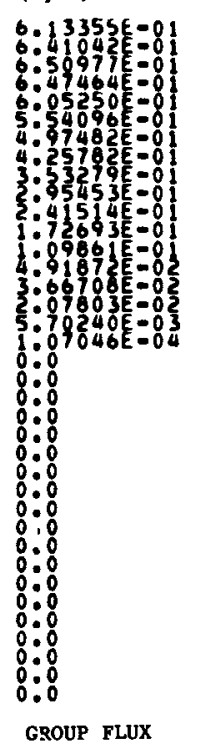

$1.49959 E^{2}-8$
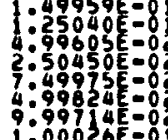

1:98936 =

$1: 99046=0$

1:49999

$1: 50006$

3:33383E=0

5: $000995=0$

3:

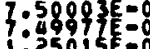

::ibs

:49995 E- 0

$1: 5608$

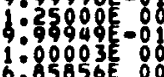

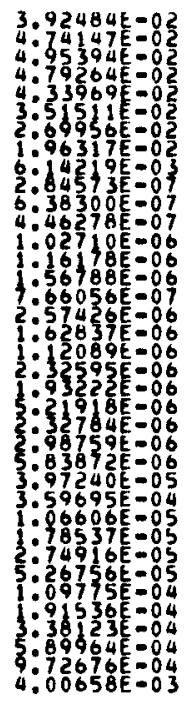

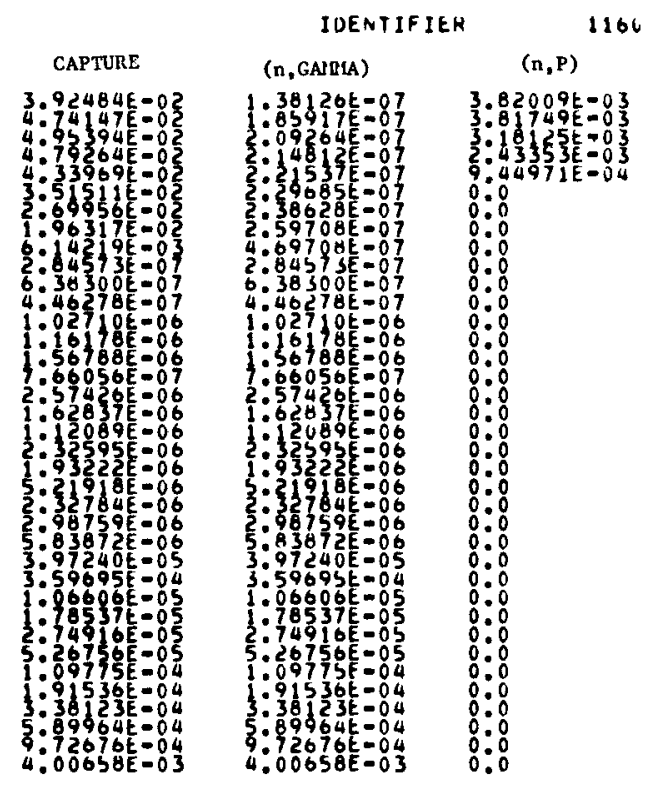


PHOTON INTERACTION CROSS SECTIONS FOR B-11
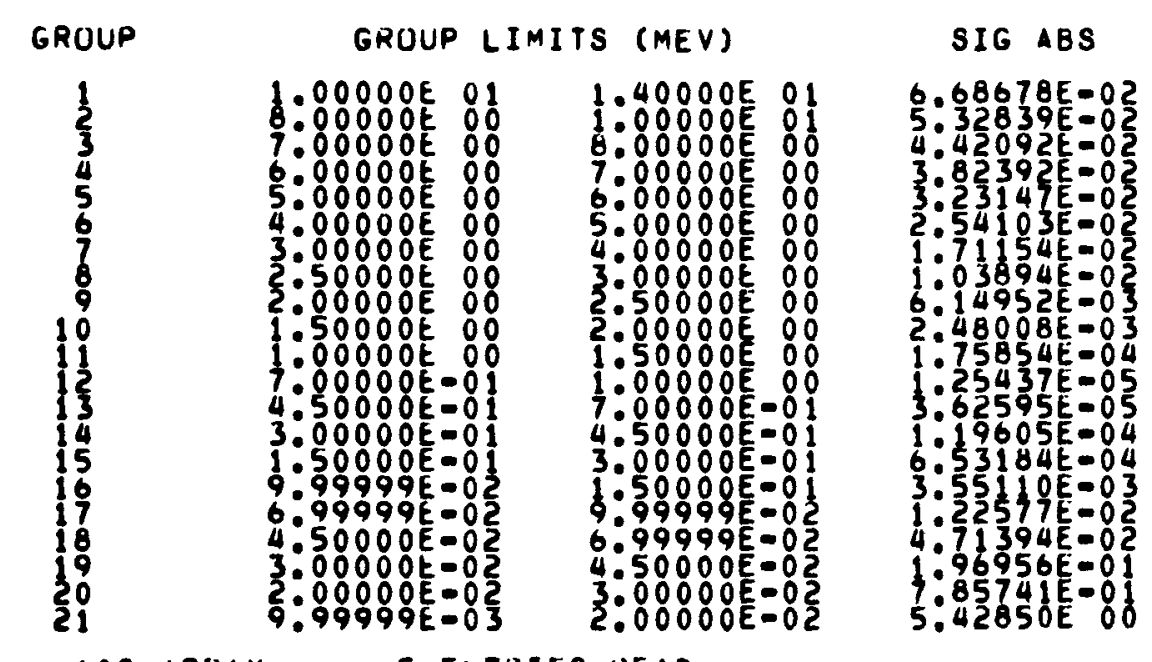

SIG PE

SIG PP

ENERGY DEP
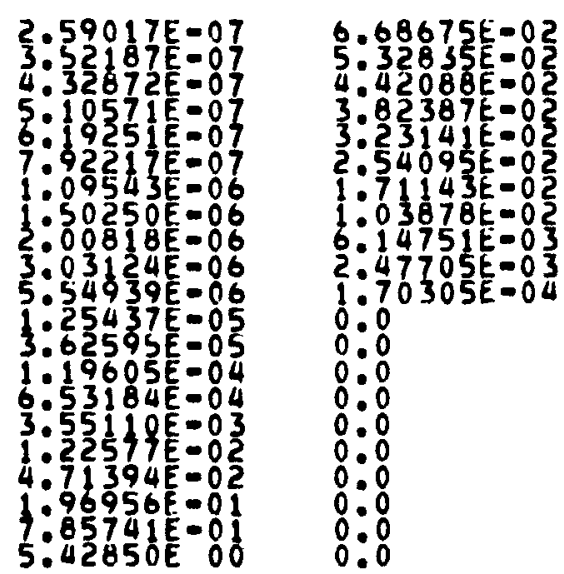

2:59724E 00 1:04270 800 107439600 1: $32383 E$ O $9.64889 E-01$ $8.44980 E=0$ 列 4.04090 E $=0$ 2.820 $1: 01046 E-0$ $4.7454 E-0$ $1.69924 E-02$ $2: 24789=002$ 


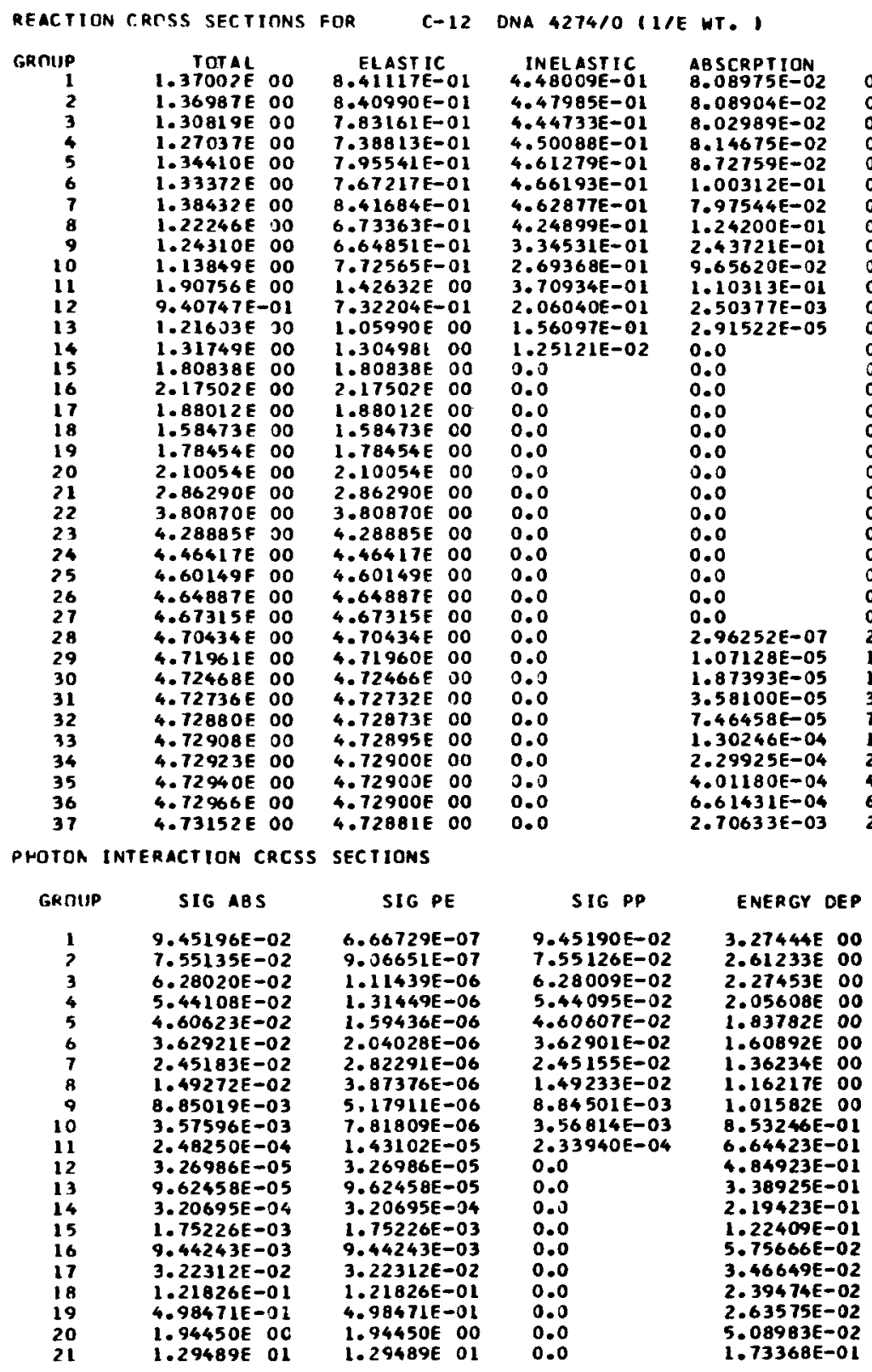

IDENTIFICATION

4274

\begin{tabular}{|c|c|c|}
\hline 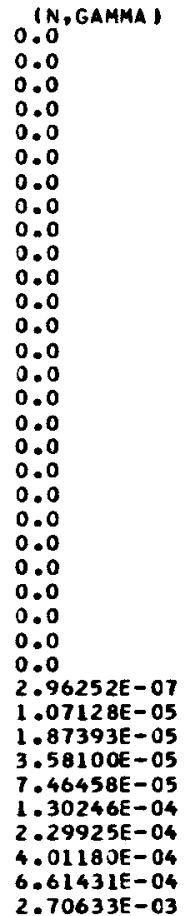 & $\begin{array}{l}1 N, A L P H \\
8.08975 E \\
8.08904 E \\
8.02989 E \\
8.14675 E \\
8.72759 E \\
1.00312 E \\
7.97544 E \\
1.24200 E \\
2.43721 E \\
9.65620 E \\
1.10313 E \\
2.50377 E \\
2.91522 E \\
0.0 \\
0.0 \\
0.0 \\
0.0 \\
0.0 \\
0.0 \\
0.0 \\
0.0 \\
0.0 \\
0.0 \\
0.0 \\
0.0 \\
0.0 \\
0.0 \\
0.0 \\
0.0 \\
0.0 \\
0.0 \\
0.0 \\
0.0 \\
0.0 \\
0.0 \\
0.0 \\
0.0\end{array}$ & 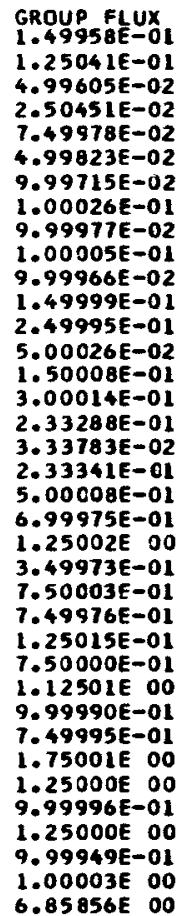 \\
\hline
\end{tabular}




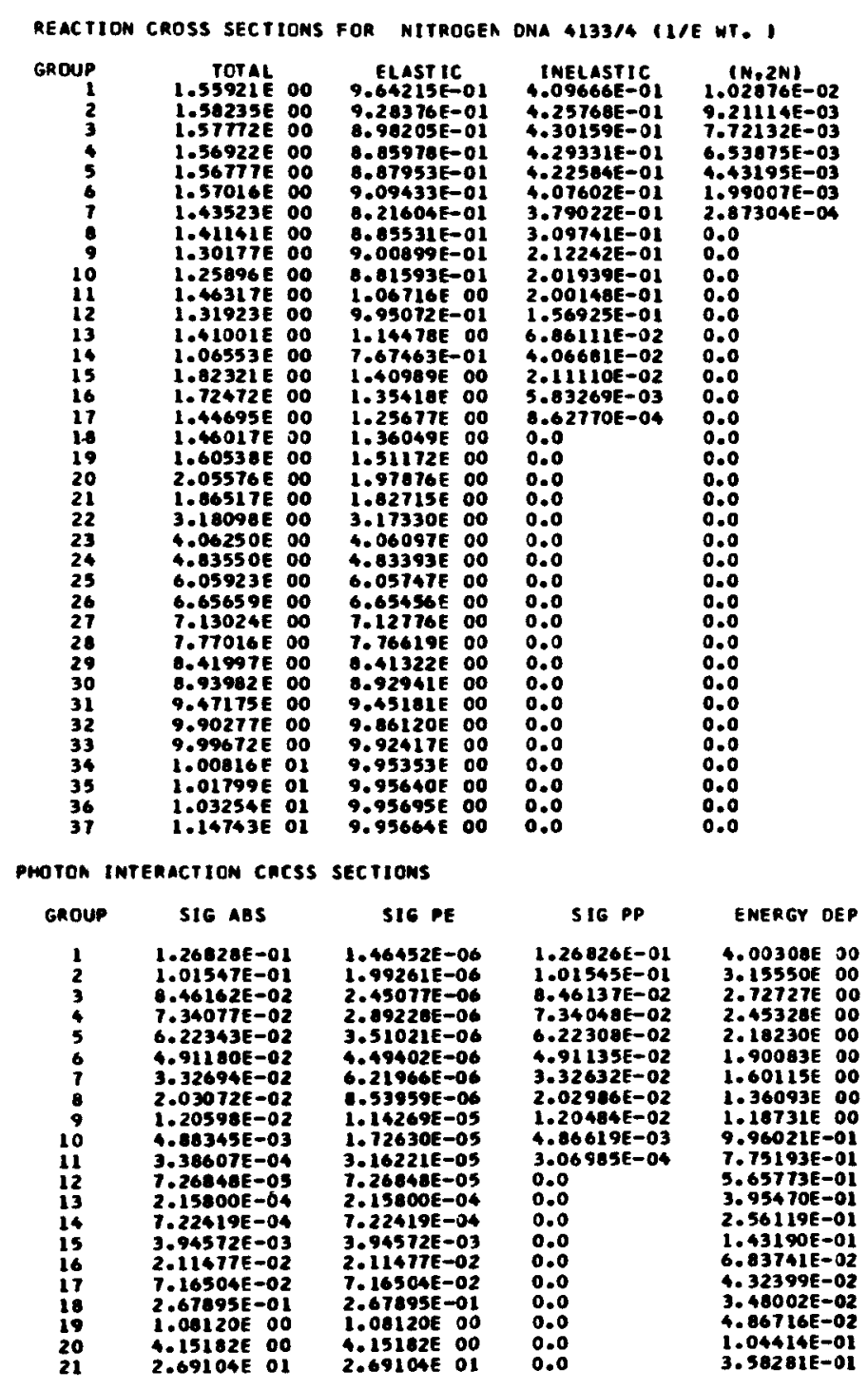

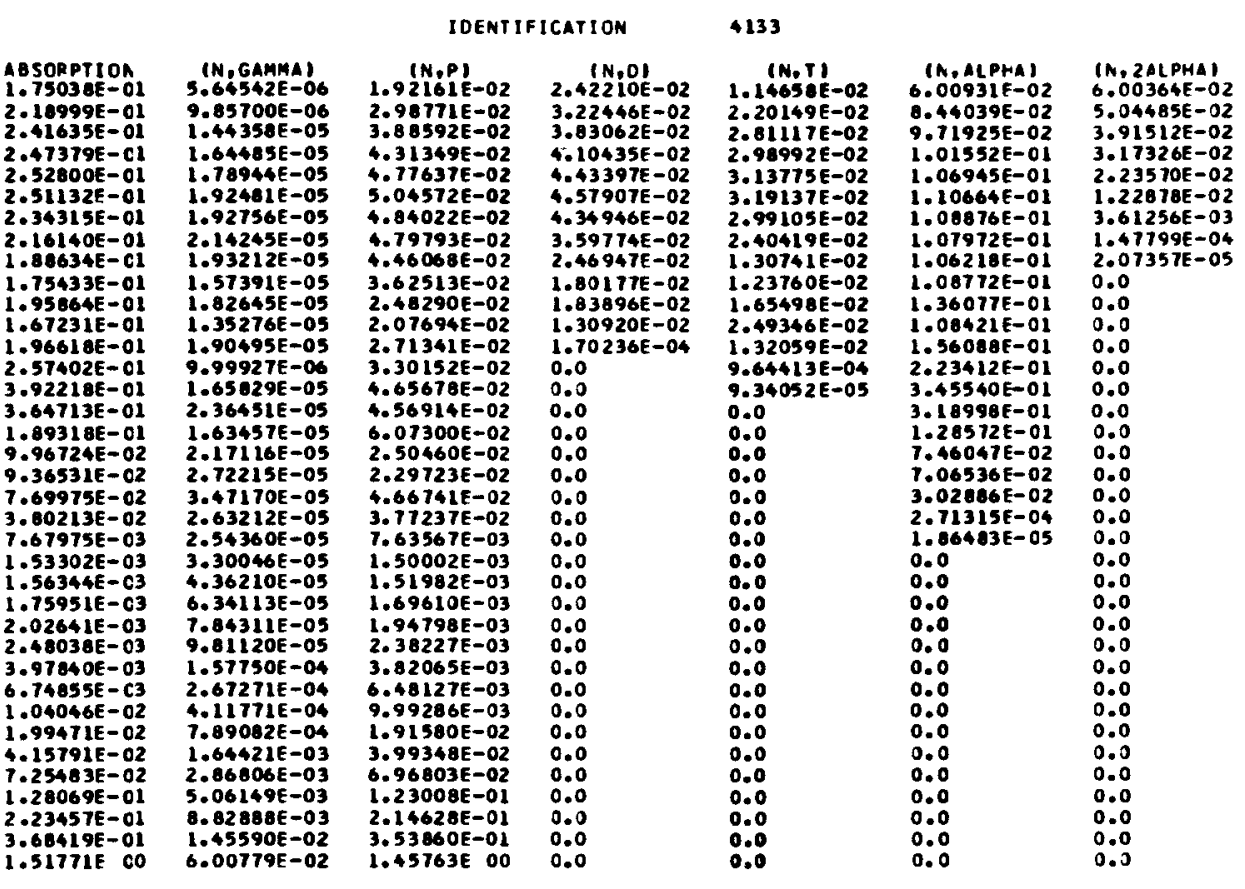




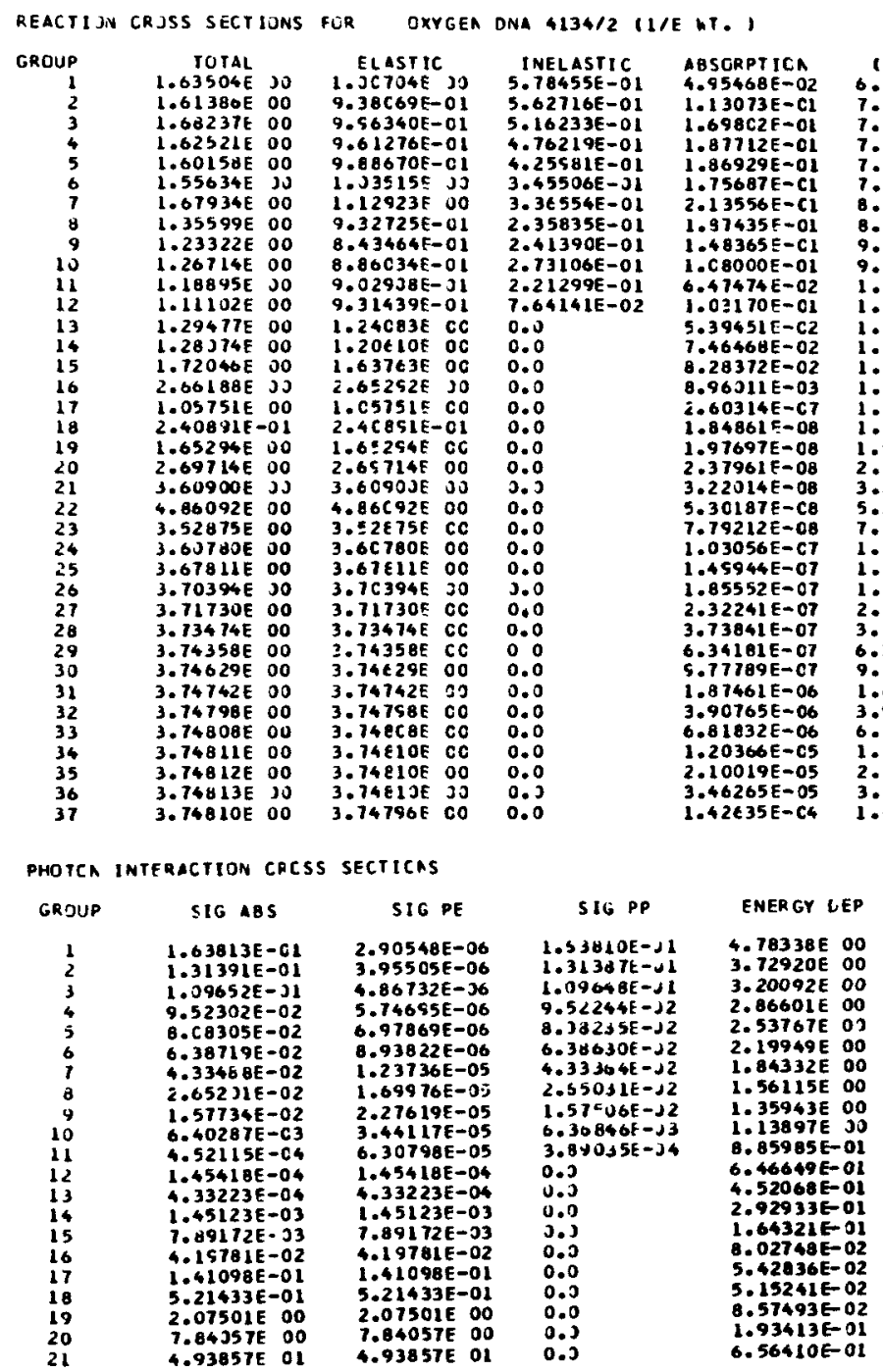


REACTION CRCSS STCTIONS FUR N 1275 ARR $37 N-716$ 30OK 10-4-74(3)

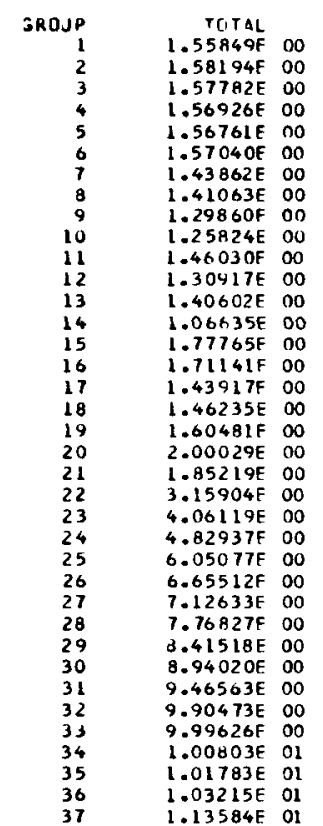

INELASTIC
$4.09279 F-01$
$4.25517 F-01$
$4.30165 F-01$
$4.29334 F-01$
$4.22732 E-01$
$4.07766 F-01$
$3.79569 E-01$
$3.11567 F-01$
$2.10959 E-01$
$2.01895 E-01$
$1.99984 F-01$
$1.53455 F-01$
$6.75016 F-02$
$4.06024 E-02$
$2.27247 E-02$
$5.46824 E-03$
$7.84385 E-04$
0.0
0.0
0.0
0.0
0.0
0.0
0.0
0.0
0.0
0.0
0.0
0.0
0.0
0.0
0.0
0.0
0.0
0.0
0.0
0.0

(N,2N) $1.03079 E-02$
$9.24378 F-03$ $9.24378 \mathrm{~F}-03$ $7.73379 \mathrm{~F}-03$
$6.54374 \mathrm{~F}-03$ $6.54374 \mathrm{E}-03$
$4.47171 \mathrm{E}-03$ $2.00550 \mathrm{~F}-03$ $3.04266 \mathrm{E}-0$ 0.0 0.0 0.0 0.0 0.0 0.0 0.0 0.0 0.0 0.0 0.0
0.0 0.0 0.0

0.0

0.0
0.0
0.0

0.0

0.0

0.0

0.0

0.0

0.0
0.0

0.0

0.0

0.0

0.0

REACTION CROSS SFCTINNS FOR N 1275 ARB 37N-21G 30OK 10-4-74(3)

$(\mathrm{N}, \mathrm{N})$
$2.40138 \mathrm{E}-02$
$3.20701 \mathrm{~F}-02$
$3.82714 \mathrm{~F}-02$
$4.10321 \mathrm{E}-02$
$4.42912 \mathrm{~F}-02$
$4.57977 \mathrm{E}-02$
$4.35613 \mathrm{E}-02$
$3.61710 \mathrm{E}-02$
$2.44603 \mathrm{E}-02$
$1.79203 \mathrm{E}-02$
$1.84109 \mathrm{E}-02$
$1.17931 \mathrm{~F}-02$
$1.58655 \mathrm{~F}-04$
0.0
0.0
0.0
0.0
0.0
0.0
0.0
0.0
0.0
0.0
0.0
0.0
0.0
0.0
0.0
0.0
0.0
0.0
0.0
0.0
0.0
0.0
0.0
0.0
0

(N, T)

$1.12552 \mathrm{E}-02$ $2.17863 F-02$ 2.8085 YE-02 Balle-02 3.1367 DE -02 $3.19073 F-02$
$2.9960 \mathrm{BE}-02$ $2.4194 \mathrm{OE}-02$ $1.28779 E-02$ $1.24318 E-02$ $1.65312 \mathrm{E}-02$ $1.28351 E-02$ $9.51306 E-04$ $1.14611 \mathrm{LE}-04$ 0.0 0.0
0.0
0.0
0.0 0.0

0.0

0.0

0.0

0.0

0.0

0.0

0.0

0.0

0.0

0.0

0.0
0.0
0.0

0.0

$(N, A L P H A)$
$5.94708 E-02$
$8.39491 E-02$
$9.71097 E-02$
$1.01527 E-01$
$1.06822 F-01$
$1.10663 F-01$
$1.08939 E-01$
$1.07953 E-01$
$1.06170 F-01$
$1.08951 E-01$
$1.36302 E-01$
$1.05035 E-01$
$1.57844 E-01$
$2.23705 E-01$
$3.41277 E-01$
$3.14810 E-01$
$1.23390 F-01$
$7.50002 E-02$
$0.93252 F-02$
$2.69923 F-02$
$2.07433 F-04$
$1.90399 F-05$
0.0
0.0
0.0
0.0
0.0
0.0
0.0
0.0
0.0
0.0
0.0
0.0
0.0
0.0
0.0

(N, 2ALPHA) $6.02458 \mathrm{~B}-02$
$5.06854 \mathrm{~F}-02$ $3.92596 \mathrm{~F}-02$ $3.17775 \mathrm{E}-02$ 2.25275E-02 $1.23537 \mathrm{~F}-02$ $3.75325 \mathrm{~F}-03$ $1.51565 \mathrm{E}-04$ $1.86032 \mathrm{~F}-05$ 1.86
0.0
0.0
0.0 0.0
0.0
0.0 0.0 0.0 0.0 0.0 0.0 0.0 0.0 0.0 0.0 0.0 0.0

0.0

0.0
0.0

0.0

0.0

0.0
0.0

0.0

0.0

0.0
IDFNTIFICATION
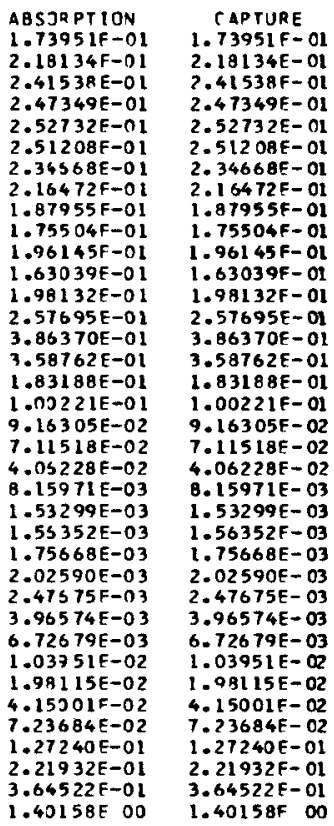

(N, GAMMA)

$\begin{aligned} & (N, G A M M A) \\ & 5.54450 F-06\end{aligned} \quad 1.89599 E-02$ $9.74150 F-06$ $1.44061 E-05$ $1.64346 \mathrm{E}-0 \mathrm{~S}$ $1.78878 E-05$ $1.92189 E-05$ $1.92940 F-05$ $2.13691 E-05$ $1.92098 F-05$ $1.5 T 172 E-05$ 1.33372E-05 $1.3372 E-05$ $1.88832 E-05$ $1.00208 E-05$ $1.60619 E-05$ $2.34835 E-05$ $1.652945-05$ $2.17758 F-05$ $2.73480 E-05$ 3. $46710 \mathrm{OE}-05$ $2.61765 F-05$ 2.54221F-05 $3.29867 F-05$ 4. $35382 E-05$ 6.32479E-OS 7.841 OOE-OS 9.797250E-04 $1.57250 E-04$ $2.66411 E-04$ 7. $7.83721 E-04$ $1.64109 \mathrm{E}-03$ $5.02873 \mathrm{~F}-03$ $0.76867 E-03$ 9.76867E-03 $1.44044 E-02$
$5.5477 T E-02$ $2.96330 \mathrm{~F}-02$ $4.31042 \mathrm{E}-02$ 4. $77063 \mathrm{~F}-02$ $5.04671 E-02$ 4. $84286 E-02$ 4. $79804 E-02$ 4. $44085 E-02$ $3.61851 E-02$ $2.48824 \mathrm{E}-02$ $2.10962 E-02$ $2.72755 E-02$ 3. $30288 E-02$ 4.49625E-02 4. $39290 F-02$ 5. $97810 \mathrm{O}-02$ $2.51987 E-02$ $2.22779 F-02$ 4.41249E-O2 $4.03892 E-02$ $8.11525 E-03$ $1.50000 \mathrm{~F}-03$ $1.51998 \mathrm{~F}-03$ $1.69344 F-03$ 1. $.94749 E-03$ $2.37879 E-03$ $6.46038 F-03$ $9.98376 \mathrm{E}-03$ $1.90278 E-02$ $3.98590 E-02$ $6.95075 E-02$ $1.22211 E-01$ 2.13163F -01 1.3461 OE OO $1.42548 \mathrm{~F}-01$ $3.10699 \mathrm{~F}-02$ $1.45153 \mathrm{E}-02$ $3.95085 F-02$ $2.34705 F-02$ 4. $0 \mathrm{~A} 405 \mathrm{~F}-02$ 3.38B 45E-02 $3.57881 \mathrm{~F}-02$ 4.38355E-02 $6.25867 \mathrm{E}-02$ $3.15687 \mathrm{E}-01$ $9.11689 E-02$ $1.90919 \mathrm{~F}-01$ $6.29880 \mathrm{~F}-01$ $8.27463 F-01$ 1.55B T8E DO $4.13802 E$ OO 8.81740F DO $1.27293 F$ OI $3.35074 \mathrm{E} \quad 00$ $7.08299 F$ OO $6.47936 F$ DO $1.02746 E$ ON $5.93486 F$ OO $8.42855 F$ OO $6.96107 F$ OO $1.07627 \mathrm{~F} 01$ $7.18708 F$ OO 5.4651 OE OD $6.26720 F$ on $4.34567 F$ DO
3.53427 OD T.RII NQF On 
REACTION CRTSSS SECTINHS FOR O 1276 ARB $37 N-21$ G 30OK 10-4-74131

SRUJP
1
2
3
4
5
6
6
7
8
9
90
11
12
13
14
15
16
17
18
19
20
21
22
23
24
25
26
27
28
29
30
31
32
33
34
35
36
37

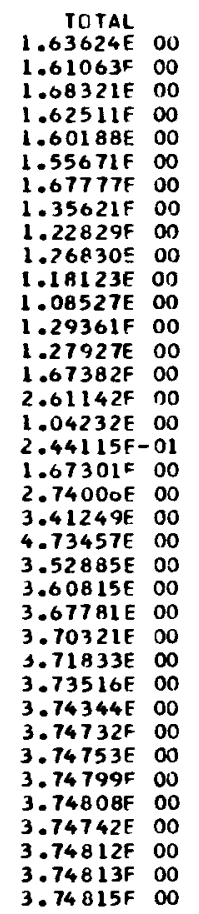

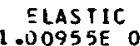
$1.00955 E$ OO $5.775476-01$ $01 \quad 5.63760 F-01$ .973 כ SE-OI $3.61122 E-01$
$9.87739 E-01$ $1.03519 \mathrm{E} 00$ $9.33241 E-01$ 3.40065E-01 $8.90221 \mathrm{E}-01$ $8.96407 E-01$ 9.1 J $1.23725 \mathrm{~F}$ OC $1.55378 \mathrm{FF} 0 \mathrm{O}$ $2.60431 \mathrm{~F} \mathrm{OO}$ $1.04232 E$ OC $2.44115 F-01$ $1.07301 \mathrm{E}$ OC
$2.74006 \mathrm{E}$ $3.41249 E 00$ $4.73457 F$ OO $3.52885 E$
$3.60815 E$ 3.6770 LE 00 3 . $3.73516 E$ OO $3.74344 E$ OO $3.74732 F \quad 00$ $3.74753 E 00$ 3.74799500 3.74471F 00 3.74815F 00
$A B S T R P T I O N$
$4.91422 E-02$ $1.10997 f-01$ $1.69561 \mathrm{~F}-01$
$1.87679 \mathrm{E}-01$ .87140F-0 2.75026F-O $1.86141 F-0$ $1.47715 \mathrm{~F}-0$ $1.05847 \mathrm{E}-0$ 6.48125E-02 $5.63665 E-02$ $7.38868 F-02$ $8.00388 E-02$ $7.11205 \mathrm{~F}-\mathrm{r}^{3}$ $2.11212 F-C$ 1.97999F-OB $2.40004 E-O B$ 3. $26812 F-O R$ $5.28237 F-08$ $7.78788 F-08$
$1.02860 E-07$ $1.49557 F-07$ 1. 85502 E-07 $2.31891 F-07$ (.72651F-07 $9.76898 \mathrm{~F}-07$ $1.86186 F-06$ 6. BOLL1E-06 $1.19586 \mathrm{~F}-05$ $2.08587 F-05$ $3.42603 \mathrm{~F}-0$ $1.31721 F-04$
IDFNTIFICATION

CAPTURF
$4.91422 F-02$
$1.19997 F-01$
$1.69561 F-01$
$1.87679 F-01$
$1.87140 E-01$
$1.75626 F-01$
$2.13975 F-01$
$1.85141 E-01$
$1.47715 F-01$
$1.05847 E-01$
$6.48125 E-02$
$1.02961 F-01$
$5.63665 F-07$
$7.39868 F-02$
$8.00388 E-02$
$7.11205 E-03$
$2.11212 E-07$
$1.84997 F-08$
$1.97999 F-08$
$2.40004 E-08$
$3.26812 F-08$
$5.28237 E-08$
$7.78788 E-08$
$1.02860 E-07$
$1.49557 F-07$
$1.85502 E-07$
$2.31891 F-07$
$3.72651 F-07$
$6.32136 F-07$
$9.76898 F-07$
$1.85186 C-06$
$3.93023 E-06$
$6.80141 E-06$
$1.19586 E-05$
$2.08587 E-05$
$3.42603 F-05$
$1.31721 E-04$

(N,GAYMA)
$6.62171 E-09$
$7.09625 E-09$
$7.47034 E-09$
$7.56161 E-09$
$7.75049 F-09$
$7.99834 E-09$
$8.29950 E-09$
$8.72502 E-09$
$9.19085 F-09$
$9.66207 F-09$
$1.01445 E-08$
$1.08361 F-08$
$1.19642 F-08$
$1.28672 F-08$
$1.34772 E-08$
$1.57555 F-08$
$1.73688 F-09$
$1.84981 E-C 8$
$1.97999 F-08$
$2.40004 E-08$
$3.26812 F-08$
$5.28237 F-08$
$7.78788 F-08$
$1.02860 E-07$
$1.49557 F-07$
$1.95502 E-07$
$2.31891 F-07$
$3.72651 F-07$
$6.32136 F-07$
$9.76898 F-07$
$1.86186 F-06$
$3.90023 F-06$
$6.80141 F-06$
$1.19586 F-05$
$2.08587 E-05$
$3.42603 E-05$
$1.31721 F-04$

\begin{tabular}{ll}
$(N, P)$ & \multicolumn{1}{c}{$(N, D)$} \\
$2.42198 F-02$ & $4.29381 F-03$ \\
$3.32387 F-02$ & $9.02038 E-03$ \\
$3.94352 E-02$ & $1.36710 F-02$ \\
$4.67642 F-02$ & $1.51898 E-02$ \\
$4.12441 F-02$ & $1.49477 E-02$ \\
$2.48181 F-02$ & $1.11937 F-02$ \\
$4.28152 E-02$ & $3.93875 F-03$ \\
$1.61836 F-03$ & $2.96184 F-05$ \\
0.0 & 0.0 \\
0.0 & 0.0 \\
0.0 & 0.0 \\
0.0 & 0.0 \\
0.0 & 0.0 \\
0.0 & 0.0 \\
0.0 & 0.0 \\
0.0 & 0.0 \\
0.0 & 0.0 \\
0.0 & 0.0 \\
0.0 & 0.0 \\
0.0 & 0.0 \\
0.0 & 0.0 \\
0.0 & 0.0 \\
0.0 & 0.0 \\
0.0 & 0.0 \\
0.0 & 0.0 \\
0.0 & 0.0 \\
0.0 & 0.0 \\
0.0 & 0.0 \\
0.0 & 0.0 \\
0.0 & 0.0 \\
0.0 & 0.0 \\
0.0 & 0.0 \\
0.0 & 0.0 \\
0.0 & 0.0 \\
0.0 & 0.0 \\
0.0 & 0.0 \\
0.0 & 0.0 \\
&
\end{tabular}

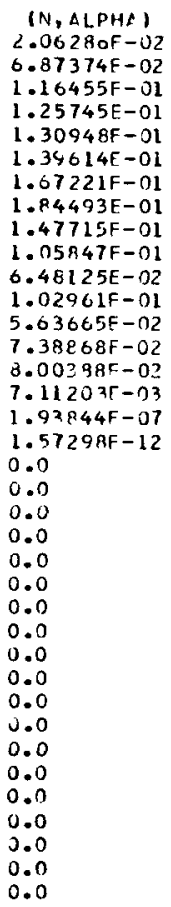

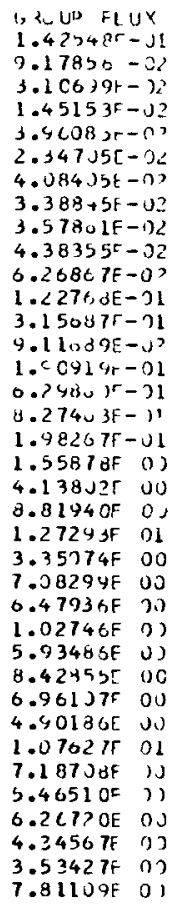




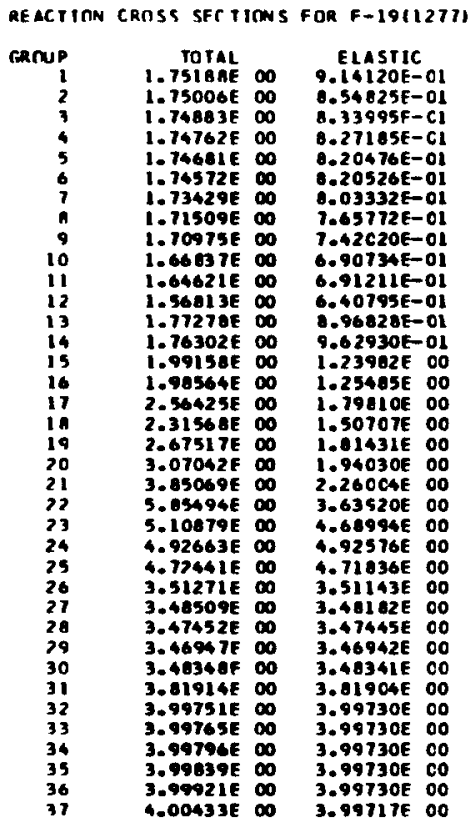

Grop
1
2
3
5
6
7
6
9
10
11
12
13
14
15
16
17
18
19
20
21
22
23
24
25
26
27
28
29
30
31
32
13
14
35
36
37

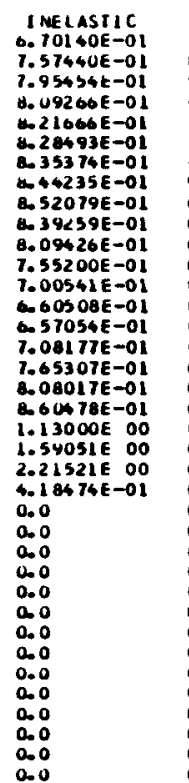

0.0

(N.ALPHA) $2.14678 E-02$ $2.34239 E-02$
$2.45407 E-02$ $2.454 d 7 E-02$
$2.53033 E-02$ $2.53033 E-02$
$3.12708 E-02$ $3.12708 E-02$
$3.88752 E-02$ $3.68752 E-02$
$4.73735 E-02$ 5. $77744 E-02$ 6. $71356 E-02$ $9.22638 E-02$ 9.22038E-02 1. $39524 E-01$ 1. $62276 E-01$ $1.38730 E-01$ 9.44 $45 E-02$ $7.927 \%$ E-04 3.28149E-OA 2. $24116 E-05$

a. 0

0.0
0.0
0.0
0.0

a.o

40

0.0

0.0

0.0

a.o

0.0

a.o

0.0
$(1,2 N)$ $.01461 f-02$ $3.88833 E-02$ $.91879 E-02$ $3.68516-02$ $4.27264 E-03$ $9.86429 E-06$ 0.0

0.0

0.0

0.0

0.0

0.0

0.0

0.0

0.0

0.0

0.0

0.0
0.0
0.0
0.0
0.0

0.0

0.0

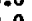

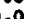

0.0

0.0

GPOUP FLUX $2.73234 E-01$
$2.27989 E-01$ $9.11688 \mathrm{E}-02$ $4.57192 E-02$ $1.36980 \mathrm{E}-01$ $9.13513 \mathrm{E}-02$ $1.83381 E-01$ $1.86053 E-01$ .91235 E-01 (.05126E-01 $3.05126 E-01$ 9.04704E-02 $2.40268 E-01$ $2.402637-01$
$4.62237 \mathrm{E}-01$ $2.90529 \mathrm{E}-01$ 4.59914 F-02 $2.78014 E-01$ $5.19522 E-01$ $5.80312 E-01$ $6.81092 E-01$ $2.18602 E-01$ 5.09076E-01 $1.13646 E-01$ $6.87156 E-01$ $1.03933 \mathrm{E} 00$ $9.73837 E-O L$ $7.60912 E-01$ 2.53351 E 00 2.58641 E 00 1.31959 e 00 $2.52906 E 00$
1.37392800 $1.93516 E 00$
7.57447000
IOENTIFICATION

1277003

ABSORPYION $5.76536 \varepsilon-02$ $6.04952 E-02$ $0.19794 E-C 2$ $6.98725 E-02$
$7.983315-02$ $9.13146 E-02$ $1.05071 E-c 1$ $1.15656 E-C 1$ $1.30380 E-C 1$ $1.45570 E-01$ $1.72139 E-01$ 1.7545e-C1 $1.39586 E-C 1$ $2.26161 E-02$ 2.26161E$5.906 B 9-C 4$ 3.86330E-04 $16435 E-04$ $1.41222 \mathrm{E}-04$ $5.34126 E-04$ $3.70525 E-C 4$ $8.73605 E-04$ $6.05034 \mathrm{E}-\mathrm{C} 3$ $1.27856 E-C 3$

$3.26367 E-03$

$6.20551 E-C 5$
$5.08849 f-05$

$5.088497-05$
$6.37127 E-05$

$1.03540 E-C 4$

$2.16712 E-04$

$6.580235-04$

$0.58023 E-04$

$1.91389 E-03$

$7.16303 E-c 3$

CAP TURE $5.276536 E-02$ $6.04952 E-02$ $6.19794 \mathrm{E}-02$ $6.90725 E-02$ $7.98531 E-02$ $.13146 E-02$ $1.05071 E-01$ $1.15656 E-01$ $1.38380 E-01$ $1.45510 e-c 1$ .72139E-01 .7stse-01 9.47058E-02 $2.26141 E-02$ 2.26141E-02 5 $3.86330 \mathrm{E}-04$ 1.16435E-04 $1.41222 E-04$ $5.34126 E-04$ $.78525 E-04$ $.73605 E-04$ . $05034 E-03$ $1.27856 E-03$ $3.26367 E-03$ $6.20551 E-05$ 5.08049E-05 $6.31127 E-05$ $1.03540 E-04$ 2.14712E-04 . $58023 \mathrm{E}-04$ $1.08549 E-03$ $1.91389 \mathrm{E}-03$ $7.16303 E-03$

(N.GAMMA) $7.69157 E-06$ $8.82221 E-06$ $1.44811 E-02$ $1.91409 E-02$ $1.11662 E-05 \quad 2.17230 E-02$ $1.20605 E-05$ 2.58077E-02 $1.33296 E-05$ 3.26168E-02 1.47226E-05 3.87907E-02 1.0275se-05 4.06437E-02 1. $2.04321 \mathrm{E}-05$ 3.21217E-02 (.) 3.19865E-05 2.28281E-04 $3.99758 E-05$ $5.962496-05$ C.0 $5.81807 E-05 \quad 0.0$ $9.40239 E-05 \quad 0.0$ $1.41222 E-040.0$ $5.34126 E-04 \quad 0.0$ $3.70525 E-04 \quad 0.0$ $8.73605 E-04 \quad 0.0$ $0.05 C 34 E-03 \quad 0.0$ $3.26367 E-C 3 \quad 0.0$ $6.20551 E-C 5$ 0.0 5.08849E-05 0.0 $6.33127 E-05$ OS 0.0 $1.035402-040.0$ 2.16712E-04 0.0 $3.580235-04$ 0.0 $1.085465-030.0$ $1.91309 E-03 \quad 0.0$ $7.16303 E-03 \quad 0.0$ 
GAMMA 1-D CROSS SECTIONS FOR F-1S(1277) 37N-21G 1/EST P-3 3OOK

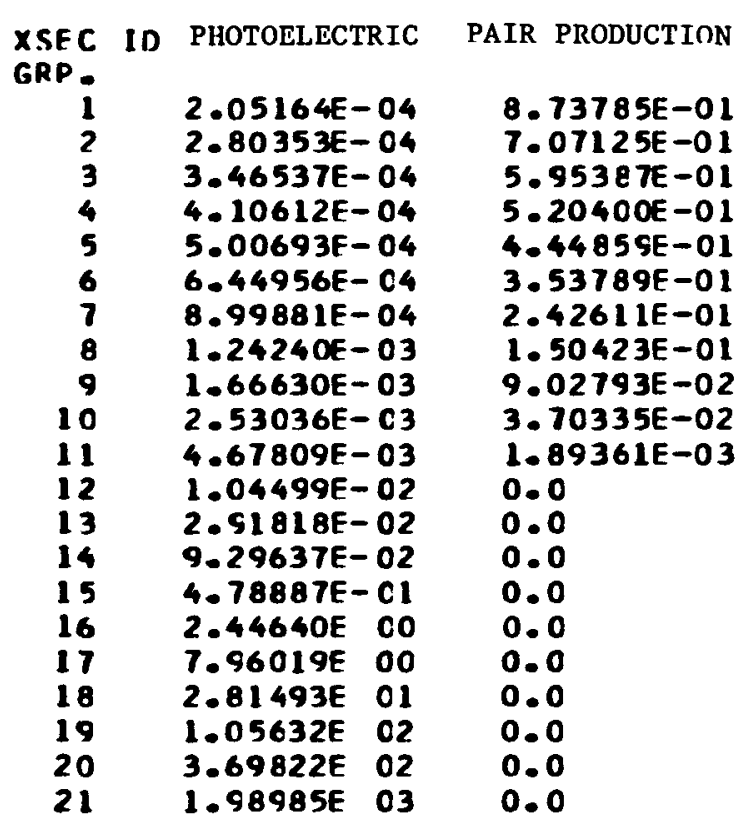

INCOHERENT SCATTERIIIG

8. $51679 E-01$

1.04787E 00

$1.19165 E 00$

1.31754E 00

$1.47812 E 00$

1.99175E 00

2.30807E 00

2.60181E 00

3. $00399 E 00$

3.60251E OO

$4.35107 E$ OO

$5.19702 \mathrm{E} 00$

6. $18788 E$ OO

7. 47431 E DO

8.86885E 00

$9.70899 E$ OO

1.04428E 01

1.1. $0815 \mathrm{E} 01$

1.15405E 01

$1.17048 E$ OI
$1.69143 E 00$
ABSORPTION

2. $05164 E-04$

2. $80353 E-04$

3. 46537 E-O4

4. $10612 E-04$

5.00693 E-04

$6.44956 E-04$

8. $99881 E-04$

1. $24240 \mathrm{E}-03$

$1.66630 \mathrm{E}-03$

2. $53036 \mathrm{E}-03$

4. $67809 E-03$

1. 04499 E-02

$2.91818 \mathrm{E}-02$

9. 29637 E-02

4. 78887 E-O

$2.44640 E$ OO

7.96019E 00

2.81493E 01

$1.05632 E 02$

3.69822 E 02

$1.98985 \mathrm{E} 03$
$I D=1277003$

TOTAI.

ENERGY ABSORPTION

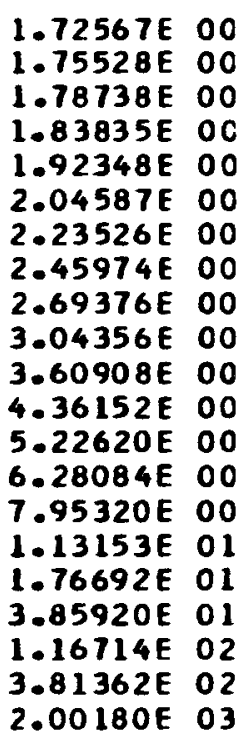

$1.67179 E 01$

$1.20253 E \quad 01$

9.77871E 00

$8.42523 E \quad 00$

$7.16612 \mathrm{E} 00$

$5.93486 E \quad 00$

4.73269E 00

3.864 C $3 E$ OO

3.29850 E 00

$2.72647 E$ CO

$2.11016 E 00$

$1.54416 \mathrm{E} 00$

1.08917E 00

$7.28023 E-01$

$4.82366 E-01$

$4.71929 E-01$

7. $56005 E-01$

$1.59344 E \quad 00$

3.82355E OO

$8.88138 E \quad 00$

$2.66030 E$ OI 
REACTIOH CRCSS SECTIONS FRR GROUP
1
2
3
4
5
6
7
8
9
10
11
12
13
14
15
16
17
18
19
20
21
22
23
24
25
26
27
28
29
30
31
32
33
34
35
36
37

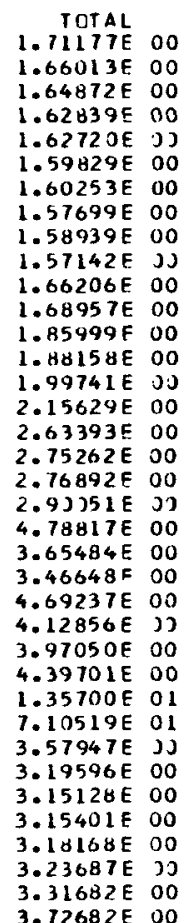

\begin{tabular}{c} 
GRUU \\
1 \\
2 \\
3 \\
4 \\
5 \\
6 \\
7 \\
8 \\
9 \\
10 \\
11 \\
12 \\
13 \\
14 \\
15 \\
16 \\
17 \\
18 \\
19 \\
20 \\
21 \\
\hline
\end{tabular}

SIG A?

3.025B5F-01

$2.43642 E-01$

$2.34317 E-J 1$
$1.77613=-01$

1. $511595-01$

$1.19751 \equiv-0$

8. $26195 E-02$
$5.32325 E-32$

$2.59837 F-02$

$1.22834 E-02$
$1.00480 E-03$

$7.17367 F-04$

2. 373$) 6 E-J 3$

$6.77450 E-03$

1.609065-01

6. $34248 F-D 1$

$2.32643 \mathrm{E} 3 \mathrm{C}$

$9.16045 E$ OC

$3.40911 \mathrm{~F}$
$2.07232 \mathrm{E}$ SIG PE

ELASTIC
8.57734 E
$8.31903 E-01$ $8.31903 \mathrm{E}-01$ $1.81860 E-01$ $7.49938 \mathrm{E}-01$
$6.77498 \mathrm{E}-01$ $6.45917 \mathrm{E}-01$ 5.91756 E- 01 $5.91756 E-C 1$
$0.04256 E-C I$ $6.83147 \mathrm{E}-01$ $7.39451 \mathrm{E}-\mathrm{Cl}$ $9.40102 E-01$ 1. 1. 348638 E 00 2.07815E 00 $2.16854 E$ OO
$2.36026 E$ JO $4.4324 \mathrm{EE} 00$ $3.46555 \mathrm{E}$ CO $4.69 C 82 E 00$ .12619 U J $.97 C 28 E \mathrm{CO}$ $4.39674 E$
$1.35695 \mathrm{CO}$ $7.10519 E$ OI $3.57129 E J 0$ . $135 C 8 F$ CO $3.13351 E C O$ $3.14557 E$ CO $3.17387 \mathrm{E}$ OO $.21295 \mathrm{~F} 00$

$1.43816 \bar{C}-05$ $2.41589 E-35$ $3.47322 E-05$ $4.45335 E-J 5$ $6.16915 E-05$ 1. $12417 E-04$ $3.16628 E-04$ $7.17367 e-04$ 2. $) 7326 E-J 3$ 6.77म5, 1. $959 C 6 E-01$ $6.34248 E-01$ $2.32643 \mathrm{E})$ $9.16095 E$ OO $3.4 C 911 E$ OI
$2.07232 E$ O2
ONA 4156/1 (1/E WT. )

INELASTIC
$6.26258 E-01$
$6.01372 E-01$
$6.18441 E-01$
$6.40423 E-01$
$6.58517 E-J 1$
$7.02368 E-01$
$7.40030 E-01$
$7.82432 E-01$
$8.41950 E-01$
$8.74343 E-J 1$
$8.99525 E-01$
$9.072 C 4 E-01$
$9.01836 E-01$
$8.59420 E-01$
$8.582 C 7 E-31$
$8.07432 E-01$
$6.53652 E-01$
$6.74278 E-01$
$6.00187 E-01$
$5.40042 E-J 1$
$3.55397 E-01$
$1.98533 E-03$
0.0
0.0
0.3
0.0
0.0
0.0
0.0
3.3
0.0
0.0
0.0
0.0
0.3
0.0
0.0

sis

$3.02571 \mathrm{~F}-01$ $3.02571 F-01$
$2.43623=-01$ $2.33593 E-J 1$
$1.77585 E-01$ $1.511245-0$ $1.19706=01$ $8.15578 E-02$
$5.01176 E-22$ $2.98699=-02$ $1.21109 E-02$ $6.88176=-04$ 0.0 1.3 0.0 0.0 0.0 0.0 0.0
IDEN IFICATION

4156

$(N .2 N)$
$5.21882 E-02$
$5.05461 E-02$
$3.51635 E-02$
$2.02620 E-02$
$6.33841 E-) 3$
0.0
0.0
0.0
0.0
0.0
0.0
0.0
0.0
0.0
0.0
$C .0$
0.0
0.0
0.0
0.3
0.0
0.0
0.0
0.0
0.3
0.0
0.0
0.0
0.0
0.0
0.0
0.0
0.0
0.0
0.3
0.0
0.0

ABSOFPIIUA $1.70308 \mathrm{~F}-01$
$1.76 \mathrm{C} 78 \mathrm{E}-\mathrm{Cl}$ $1.05838 t-c 1$ $2.024 J 3 E-C 1$ .16587F-Cl $2.02304 E-C 1$ 1. $7.93895 E-02$ $4.291695-02$ $1.20539 E-02$ $2.10130 F-C^{-3}$ 1.75689e-C4 $1.777125-C_{4}$ $1.83994 E-C 4$ $1.90562 E-C 4$ 2. $) 9875 E-04$ $2.88827 \mathrm{E}-\mathrm{CL}_{4}$ $9.48597 \mathrm{E}-\mathrm{C}_{4}$ $2.38 .017 E-C 3$ $2.25910 F-C^{4}$ $2.71073 E-C_{4}$ $4.06363 E-04$ $1.20008 E-C 6$ (8.18335F$1.21963 F-02$ $2.05047 F-C 2$ $3.61164 \mathrm{E}-\mathrm{C2} 2$ $0.30393 E-32$ $1.03874 \mathrm{E}-\mathrm{Cl}$
$2.40065 E-04$ $2.32765 E-J 4$
$2.22034 \mathrm{E}-\mathrm{O}$
$2.22545 \mathrm{~F}$ $2.12545 \mathrm{~F}-04$ $2.013065-04$ $1.82178 f-04$ 1. $17478 \mathrm{~F}-04$ $1.71974 E-04$ $1.69626 E-04$ $1.67336 E-34$ $1.638191-04$ 1.61247E-04 $1.77712 \mathrm{E}-34$ $83994 E-04$ $2.90562 t-04$ $2.38827 E-04$ $5.499375-34$ $28597=-04$ $2.25910 E-04$ $2.71073 E-34$ 1.6083e-04 $8.18335 E-03$ $1.21963 \mathrm{E}-12$ $2.05047 \mathrm{E}-02$ $3.61164 E-02$ $6.30090=-02$ $1.03874 \mathrm{~F}-01$

\begin{tabular}{|c|c|c|}
\hline$(n, p)$ & & 10 CAPTURE \\
\hline $\begin{array}{l}4.48313 F-02 \\
4.35277 F-J 2\end{array}$ & $1.30473 f-91$ & 0.0 \\
\hline $\begin{array}{l}4.351713 F-02 \\
4.413\end{array}$ & $\begin{array}{l}1.26547 F-11 \\
1.31684 \mathrm{~F}-01\end{array}$ & 0.0 \\
\hline $4.58509 \mathrm{E}-02$ & $1.397755-01$ & 0.0 \\
\hline $5.24414 E-02$ & $1.49761 E-01$ & 0.0 \\
\hline $5.05359 \mathrm{E}-02$ & $1.59692 \mathrm{e}-01$ & 0.0 \\
\hline 6.4$) 946 E-32$ & $1.5231 \mathrm{JF}-\mathrm{J1}$ & 0.0 \\
\hline $8.52655 \mathrm{~F}-\mathrm{C} 2$ & $1.16861 E-01$ & 0.0 \\
\hline $5.94929 E-02$ & $8.35201 F-02$ & 0.3 \\
\hline $5.40756 c-02$ & $5.36370 F-02$ & 0.0 \\
\hline $5.04652 F-02$ & $2.07546 E-02$ & 0.0 \\
\hline $3.58360 F-32$ & $6.94291 F-33$ & 0.0 \\
\hline $1.69547 \mathrm{E}-02$ & $9.35359 \varepsilon-04$ & 0.0 \\
\hline $1.53006 \mathrm{~F}-03$ & $4.09996 F-04$ & 0.0 \\
\hline $4.38835 F-04$ & $1.76721 E-04$ & 0.0 \\
\hline $1.3171 \cap E-05$ & $1.38376 E-07$ & 0.0 \\
\hline 3.5 & 3.3 & 0.0 \\
\hline 0.0 & 0.0 & 0.0 \\
\hline 0.0 & 0.0 & 0.0 \\
\hline 0.0 & 0.0 & 0.0 \\
\hline 0.0 & 0.0 & 0.0 \\
\hline 1.3 & 3.) & c.o \\
\hline 0.0 & 0.0 & $1.30181 E-03$ \\
\hline 0.0 & c.o & 0.0 \\
\hline 0.0 & 0.0 & 0.0 \\
\hline & 0.0 & 0.0 \\
\hline & J. & \\
\hline 0.0 & 0.0 & $5.55632 \mathrm{E}-03$ \\
\hline 0.0 & 0.0 & $8.05572 \mathrm{~F}-02$ \\
\hline 0.0 & 0.0 & $2.91871 E-04$ \\
\hline 0.0 & 0.0 & 0.0 \\
\hline J.3 & 0.3 & 0.0 \\
\hline 0.0 & 0.0 & c. 0 \\
\hline & c.o & 0.3 \\
\hline & 0.0 & 0.0 \\
\hline 0.0 & 0.0 & 0.0 \\
\hline 3.) & 2.3 & 0.0 \\
\hline
\end{tabular}

HAERCY OFP

$7.43155 E$ OO 5.63239800 4.146355 SOA $3.66516 \mathrm{~F}$ vo $3.13622 E$ OD $\begin{array}{ll}2.59006 C & 00 \\ 2.17961 E & J\end{array}$ 1.87969600 $1.56893 \mathrm{E} 00$ 1.21858500 $8.8957) E-01$ 4. C4507E-31 $2.309855-01$ $1.10820 \mathrm{E}-01$ $1.58694 \mathrm{~F}=11$ $3.442665-01$ $8.23763 \mathrm{~F}-01$
$2.75091 \mathrm{~F}$ no 


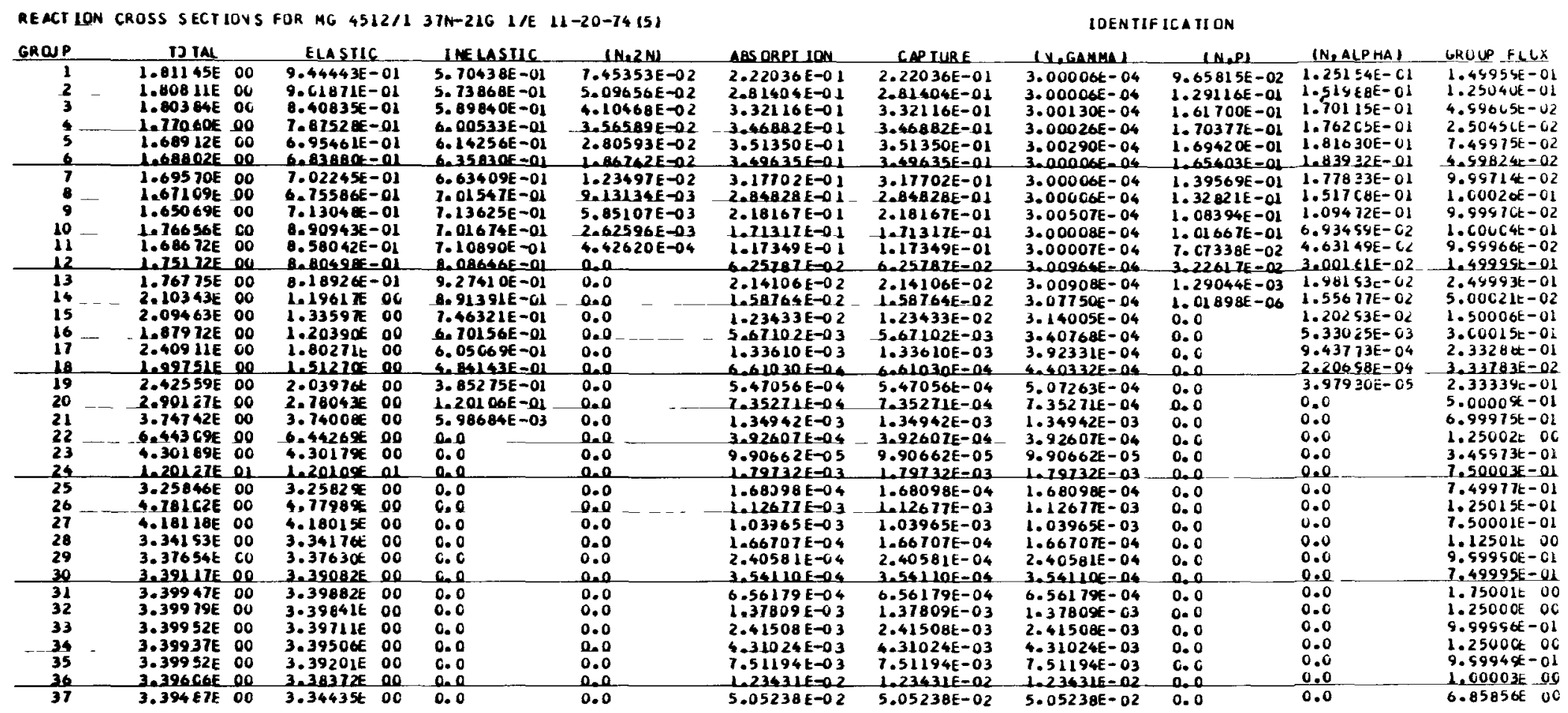


PHOTON INTERACTION CROSS SECTIONS FOR Mg

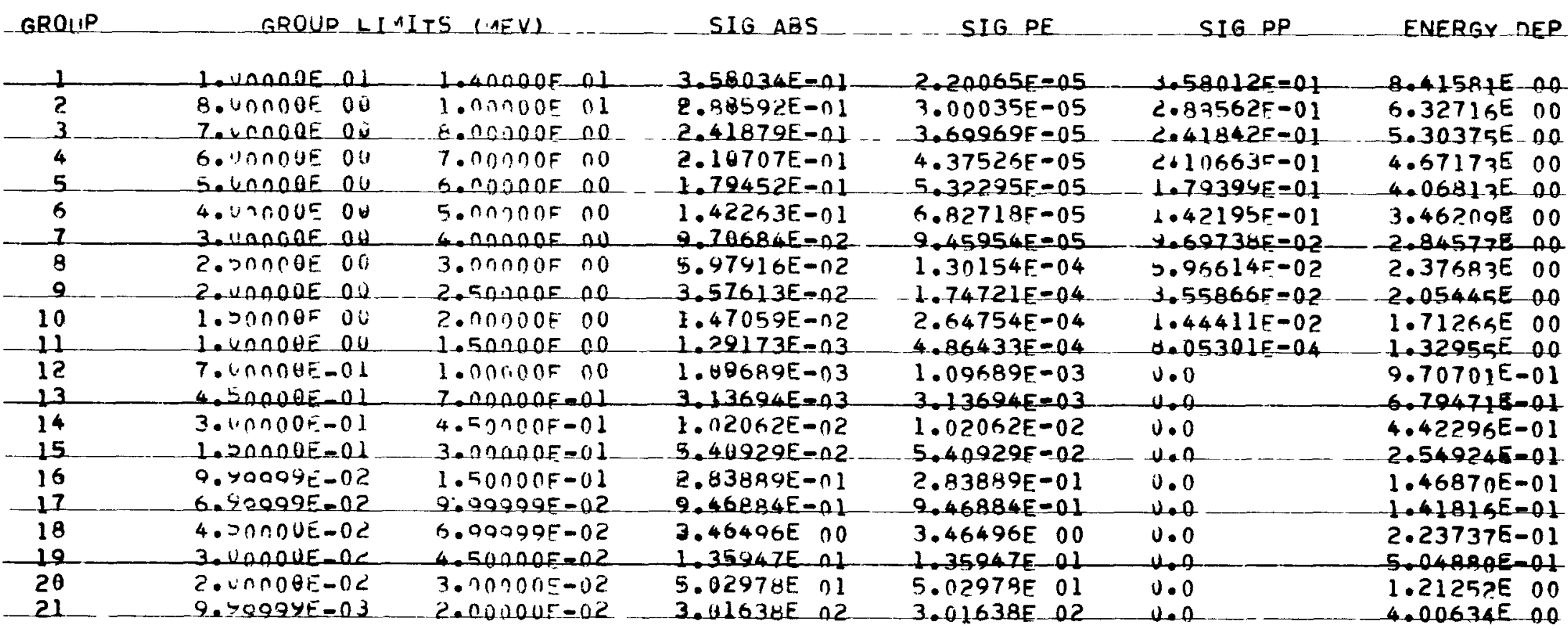


REACTIIN CRUSS SECTIUNS FJR ALUMINUM ONA $4135 / 3$ 11/E WT.

\begin{tabular}{|c|c|c|c|c|}
\hline $\begin{array}{r}\text { GROUP } \\
1 \\
2 \\
3 \\
4 \\
5 \\
6 \\
7 \\
8 \\
9 \\
9 \\
10 \\
11 \\
12 \\
13 \\
14 \\
15 \\
16 \\
17 \\
18 \\
19 \\
20 \\
21 \\
22 \\
23 \\
24 \\
25 \\
26 \\
27 \\
28 \\
29 \\
30 \\
31 \\
32 \\
33 \\
34 \\
35 \\
36 \\
37\end{array}$ & 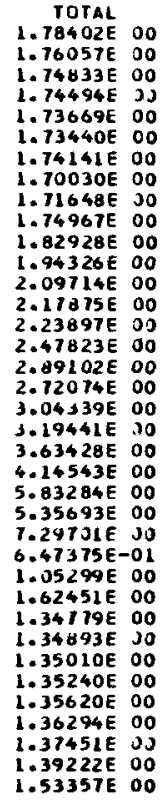 & 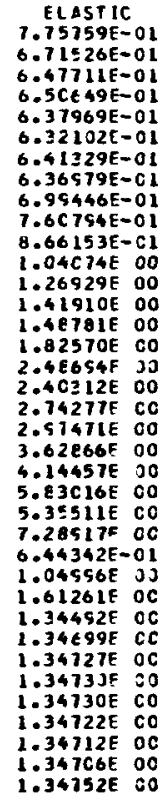 & 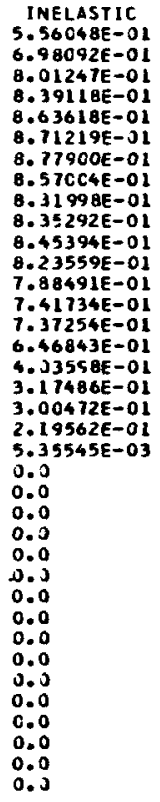 & $\begin{array}{l}3.09,2 N) \\
3.06919 E-01 \\
1.94352-01 \\
7.76125 E-02 \\
2.63054-02 \\
2.00575 E-02 \\
0.3 \\
0.0 \\
0.0 \\
0.0 \\
0.0 \\
0.0 \\
0.0 \\
0.0 \\
0.0 \\
0.0 \\
0.0 \\
0.0 \\
0.0 \\
0.0 \\
0.0 \\
0.0 \\
0.0 \\
0.0 \\
0.0 \\
0.0 \\
0.0 \\
0.0 \\
0.0 \\
0.0 \\
0.0 \\
0.0 \\
0.0 \\
0.0 \\
0.0 \\
0.0 \\
0.0 \\
0.0\end{array}$ \\
\hline
\end{tabular}

IDEN IFICATION

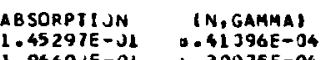
A $45291 E-J 1$ $2.21755 E-01$ $2.3309 b E-01$ $2.22179 E-01$
$2.0315 E-U 1$
$1.05039 E-01$ $11.53565 E-01$ .89695E $=02$ $33.93635 E=02$ $1.79161 E-02$
$5.6918 E-02$
$5.09733 E-03$ $.6973 D E-03$
$. .76804 E-J 4$ 1. $46792 E-\infty$ 1. $38807 E-04$ $2.61248 E-04$
$8.5695 E-J 4$ $2.6875 \mathrm{TE}-03$
$1.01700 \mathrm{E}-03$ 7. $84610 E=03$ $3.02695 \mathrm{E}-\mathrm{J3}$ $1.18923 E=02$ $1.94571 E-03$

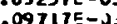
4.5157AE-02 $3.27361 E-04$
$3.00342 E-04$ $4.65936 E-04$
$\because .25197 E-04$ $3.79650 E-04$
$3.23903 E-04$ \begin{tabular}{l}
$2.73452 E-04$ \\
\hdashline $.27938 E-04$
\end{tabular} $1.40677 E-04$ $1.83470 E-05$ $3.94740 E-05$ ..35848E-05

$\therefore 44695 E-04$ 1.38807 F $=4$ (549)5E-04 作2695E-0 $.12925 E=02$ $1.94571 E-03$ $.73911 E-02$
$. \quad 51578 E-02$ $4.51578 E-02$
$1.86050 E-01$

\begin{tabular}{|c|c|c|c|}
\hline 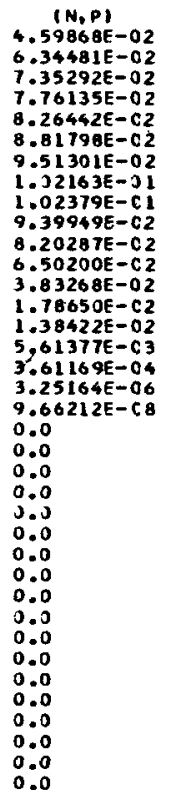 & 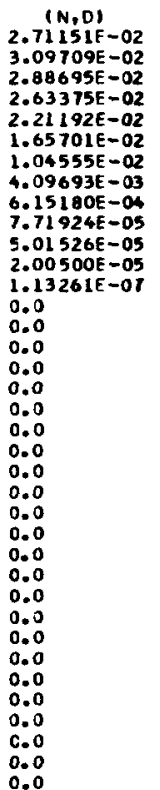 & 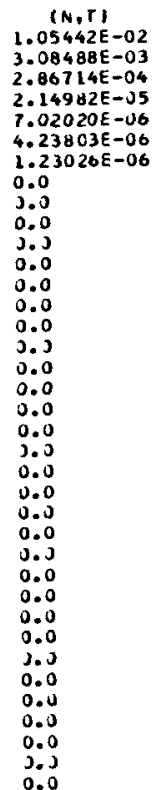 & 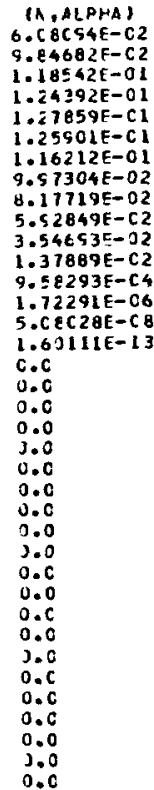 \\
\hline
\end{tabular}

CACSS SECTICAS

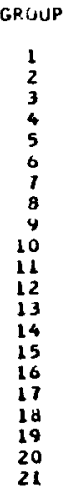

SIG AB

\begin{tabular}{|c|c|}
\hline & $\triangle I G P E$ \\
\hline 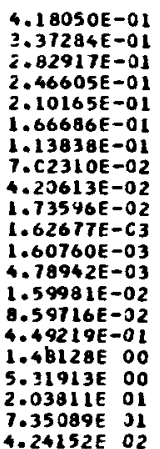 & 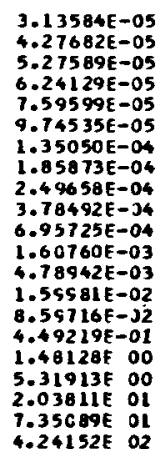 \\
\hline
\end{tabular}

SIG PP

FNERGY UEP

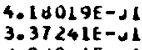

$9.45049 E$ OO
$7.05193 \mathrm{E} 00$

L.8 $2004 E-J$

2.1 JU ME-J1

$1.19703 \mathrm{E}-\mathrm{J1}$

$4=1117 E-J 2$

$0.5981 E-J 2$
$0.31069 E-J 4$

0.3
0.0
0.0

0.3

0.0

5.88126600

4.47797800

3. 194661600

$2.22907 E$ OO

1. 855659600

$1.44058 E 00$
$1.05194 E$

7. $36856 \mathrm{E}-01$
$4.00932 \mathrm{E}-01$
3

2.81632E- 111

$1.91153 \mathrm{E}-01$
$3.20053 \mathrm{E}-01$
6

3. $2805358-01$

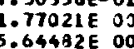




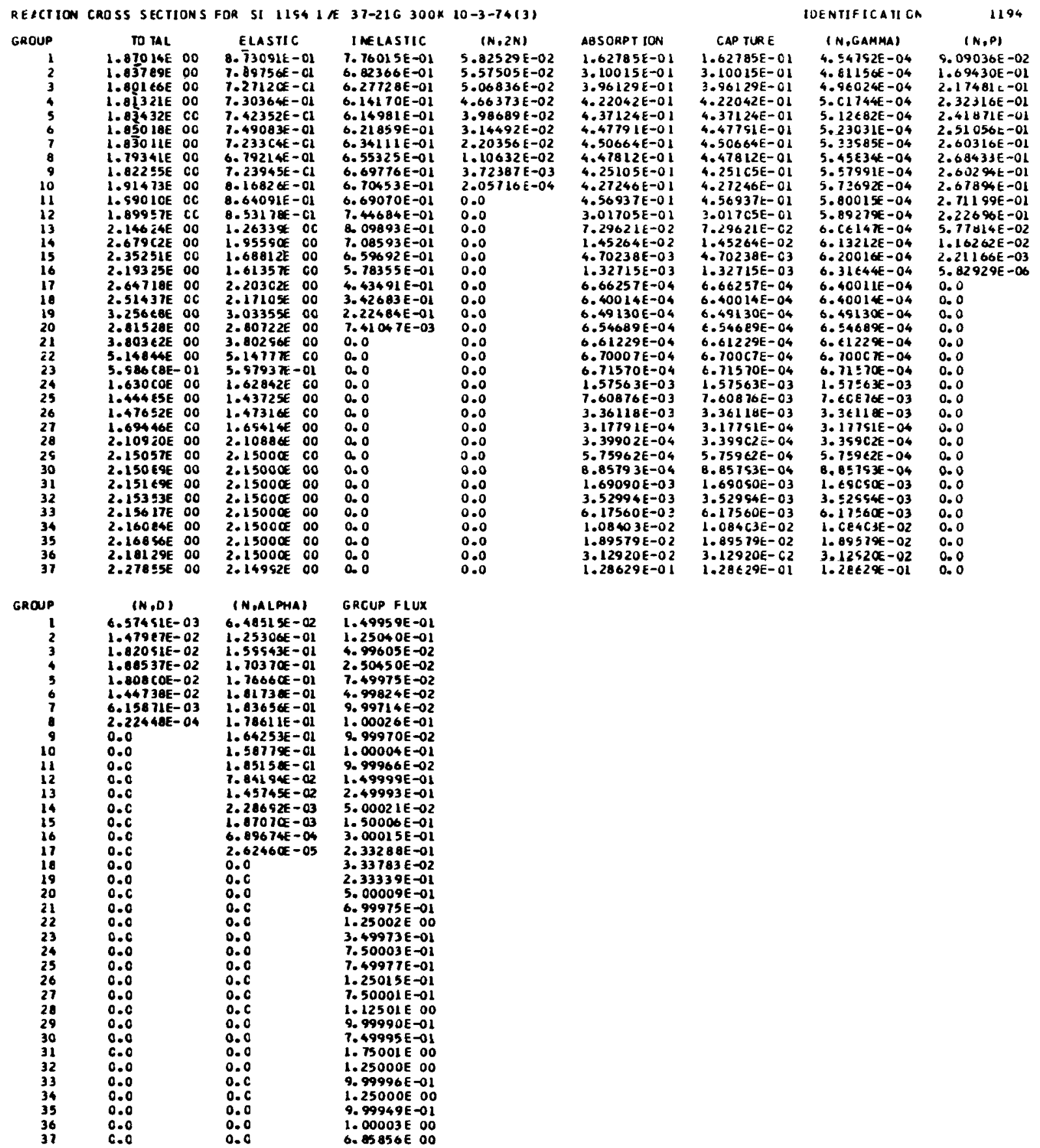


PHOTON INTERACTION CROSS SECTIONS FOR Si

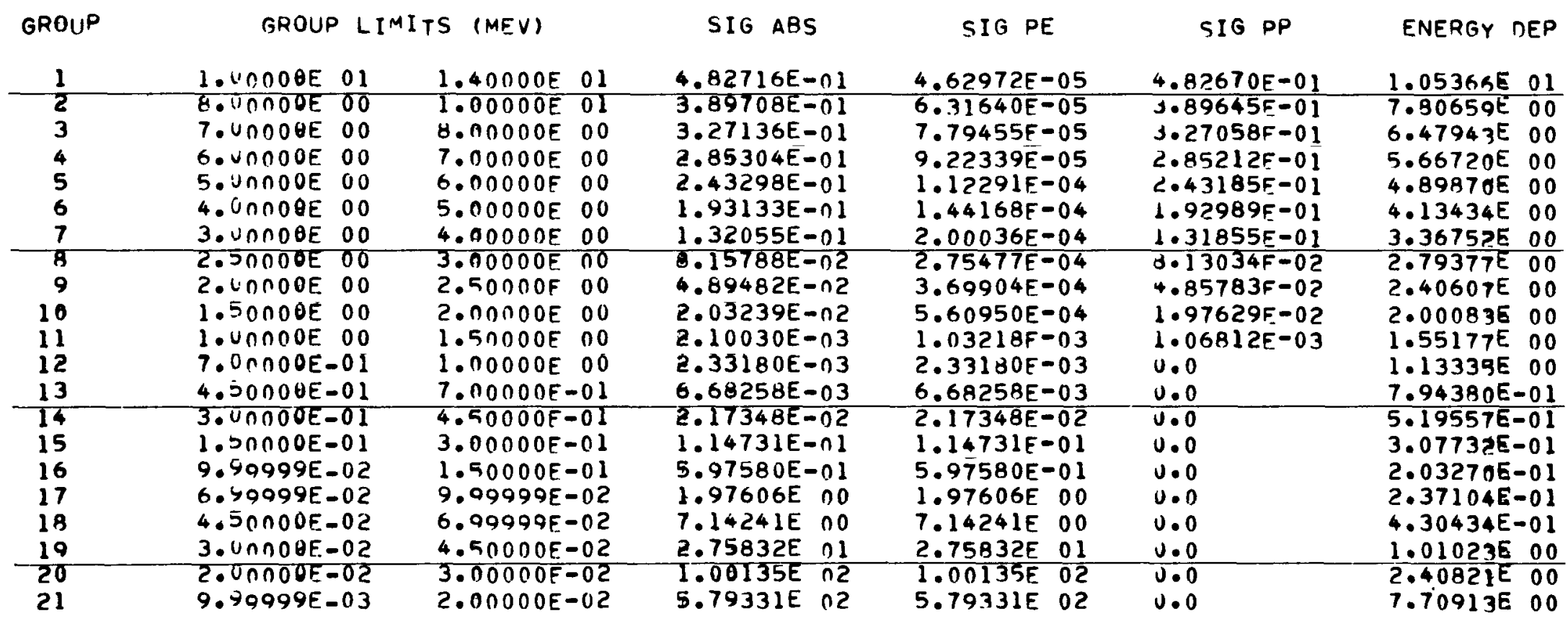


ONE DIMENSIUN AVERAGES FOR CL MAT $1149 \quad 37 \mathrm{~N}-216$ P3 $T=300 \mathrm{~K} \quad 10-9-75(3)$

\begin{tabular}{|c|c|c|c|c|c|c|}
\hline & 10 & TOTAL & ELASTIC & $\left(n, n^{\prime}\right)$ GAILIA & $(n, 2 n)$ & $\left(n, n^{\prime}\right)$ ALPHA \\
\hline $\begin{array}{r}1 \\
2 \\
3 \\
4 \\
5 \\
9 \\
8 \\
9 \\
10 \\
11 \\
12 \\
13 \\
14 \\
15 \\
16 \\
17 \\
18 \\
19 \\
20 \\
21 \\
22 \\
23 \\
24 \\
25 \\
26 \\
27 \\
28 \\
29 \\
30 \\
31 \\
32 \\
33 \\
34 \\
35 \\
36 \\
37\end{array}$ & & 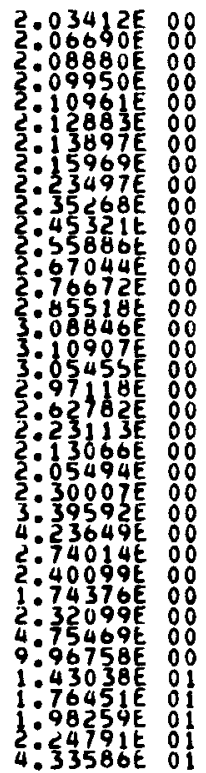 & 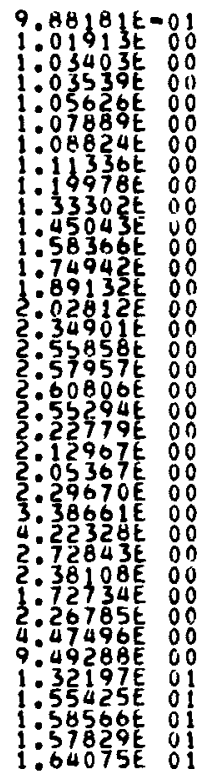 & 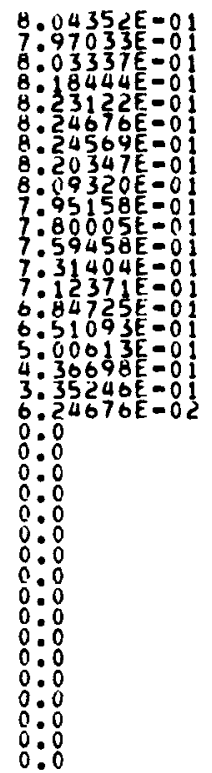 & $\begin{array}{l}3: 87909 E=02 \\
3: 4577=02 \\
2: 93481 \in=02 \\
2: 20638=02 \\
5: 73573 E=03 \\
0: 0 \\
0: 0 \\
0: 0 \\
0: 0 \\
0: 0 \\
0: 0 \\
0: 0 \\
0: 0 \\
0: 0 \\
0: 0 \\
0: 0 \\
0: 0 \\
0: 0 \\
0: 0 \\
0: 0 \\
0: 0 \\
0: 0 \\
0: 0 \\
0: 0 \\
0: 0 \\
0: 0 \\
0: 0 \\
0: 0 \\
0: 0 \\
0: 0 \\
0: 0 \\
0: 0 \\
0: 0 \\
0: 0 \\
0: 0 \\
0: 0\end{array}$ & 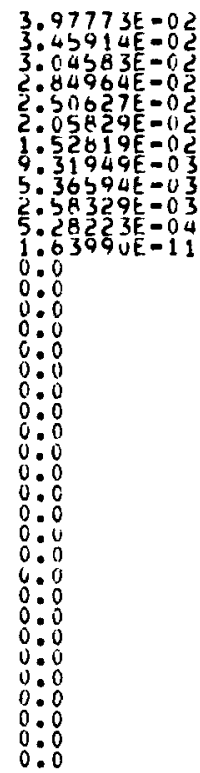 \\
\hline$x \operatorname{stc}$ & 10 & (n, GAIr $(A)$ & $(n, P)$ & $(\mathrm{n}, \mathrm{AL}, \mathrm{PHA})$ & GROUP FLJX & \\
\hline $\begin{array}{r}2 \\
3 \\
4 \\
5 \\
6 \\
7 \\
8 \\
9 \\
10 \\
11 \\
12 \\
13 \\
14 \\
15 \\
10 \\
17 \\
10 \\
19 \\
20\end{array}$ & & 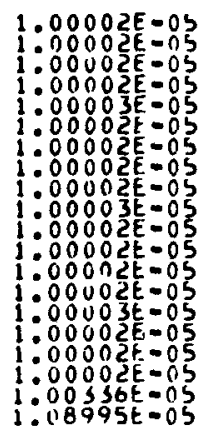 & 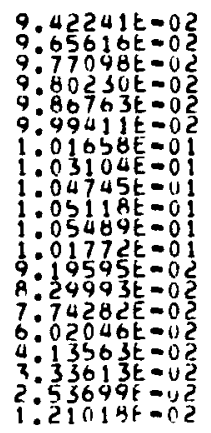 & 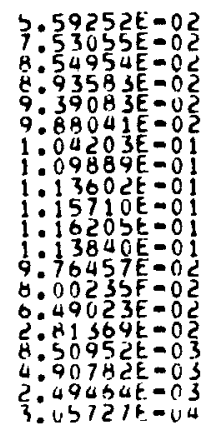 & 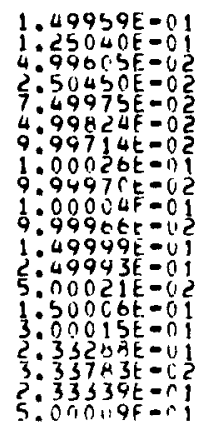 & \\
\hline
\end{tabular}

$\left(n, n^{\prime}\right) P$

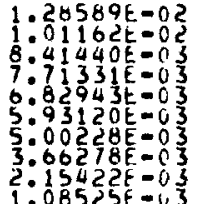

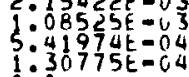

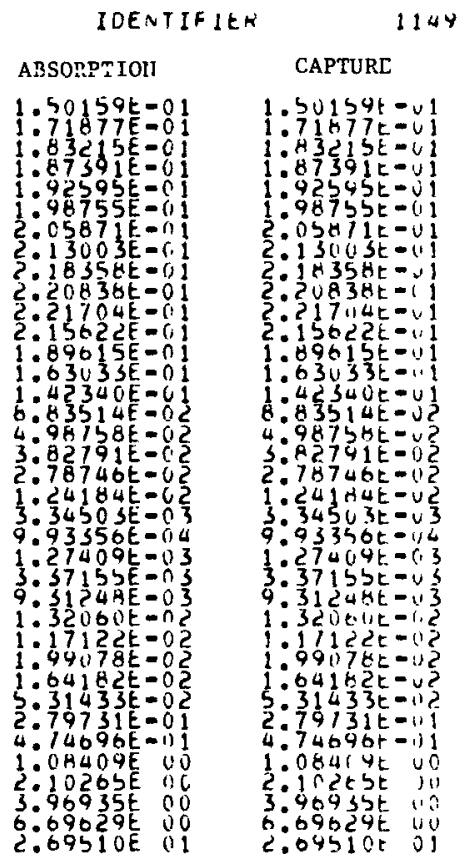


PHOTON INTERACTION CROSS SECTIONS FOR CI

\begin{tabular}{|c|c|c|c|c|c|c|}
\hline GROUP & GROUP LI & TS (MEV) & SIG AOS & SIG PE & SIG PP & ENERGY DEP \\
\hline $\begin{array}{r}1 \\
2 \\
3 \\
4 \\
5 \\
6 \\
7 \\
8 \\
9 \\
10 \\
11 \\
12 \\
13 \\
14 \\
15 \\
16 \\
17 \\
18 \\
19 \\
20 \\
21\end{array}$ & 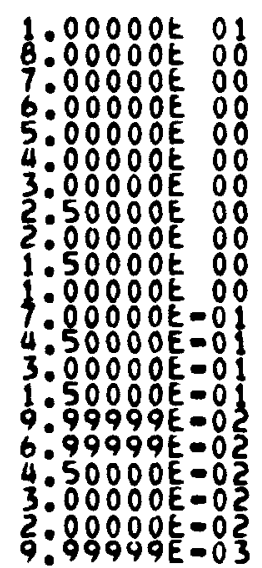 & 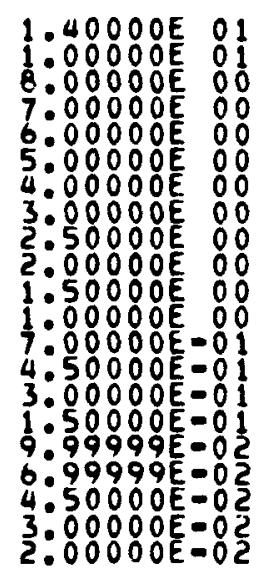 & 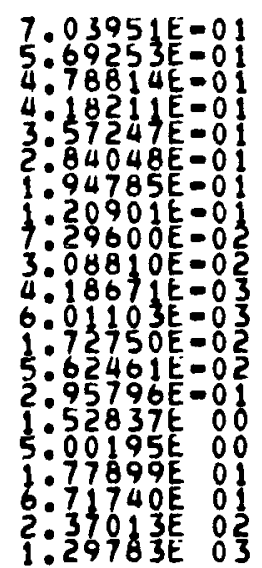 & 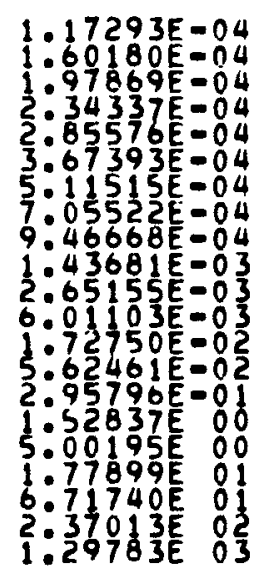 & 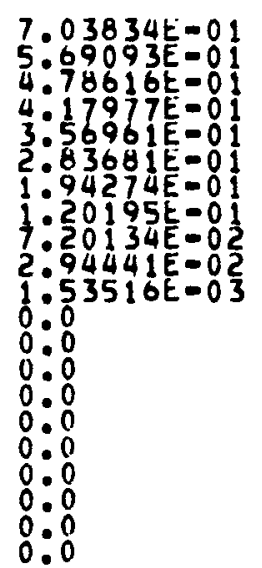 & 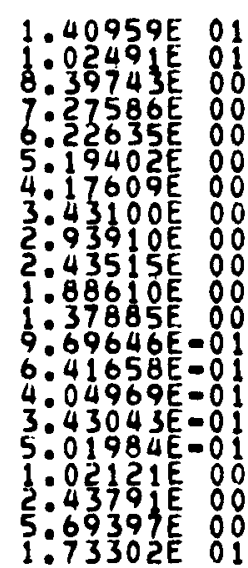 \\
\hline
\end{tabular}




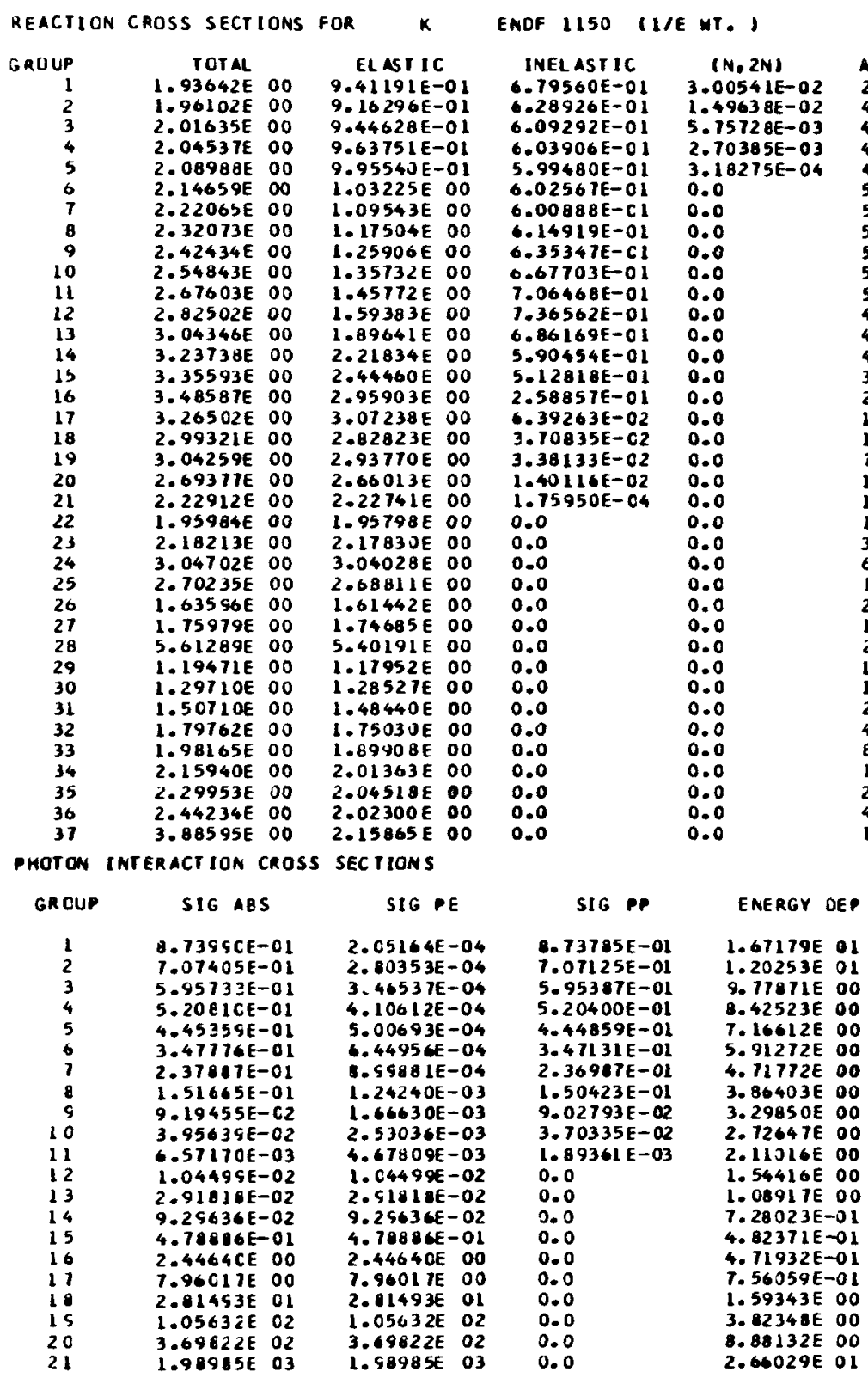

TOENT IFICATION

1150

AB SORP TI ON

2.74668E-05 $3.15062 E-05$
$3.43687 E-05$ $3.43687 E-05$
$3.56789 E-05$ $3.56789 E-05$ 3. $99314 E-05$ $4.30553 E-05$ $4.75816 E-05$ $5.25860 E-05$ $5.81167 E-05$ $6.42320 E-05$ (.) $1.03249 E-04$ $1.14202 E-04$ $1.43423 E-04$ $1.86973 E-04$ $2.43167 t-04$ $3.55121 \mathrm{E}-04$ $6.53525 E-04$ 1.81 045E-03 $3.79768 E-03$ $6.7033 \perp E-03$ $1.41907 E-02$ $1.28666 \mathrm{E}-02$ $2.10865 E-0$ $1.49900 E-02$ $1.15305 E-02$ $2.21179 E-02$ $4.61046 E-02$ $1.42012 E-01$ $2.47787 E-0$ $4.08530 E-01$
$1.68275 E$ OO (N,P) IN, NPHA) 1
$.169136719 \mathrm{NE}-01$ 3.53299E-01 $1.03343 E-01$ . $3.73662 E-01 \quad 1.20840 E-01$ $3.83261 E-01$ L $41033 E-01$ $3.80051 E-01 \quad 1.50669 E-01$ $3.73689 \mathrm{E}-01 \quad 1.56399 \mathrm{E}-01$ 3.64364E-OI $1.59283 \mathrm{E}-\mathrm{O}$ 3.51941E-OL $1.59839 E-01$ $3.12919 \mathrm{E}-0 \mathrm{~L}$ 1.47875E-OL $2.93788 E-01$ 1. $34692 E-01$ $2.80003 E-01 \quad 1.18402 E-01$ 2.11616E-OL $5.62271 \mathrm{E}-02$ 1.22927E-01 $1.21519 E-02$ $0.01209 E-02 \quad 4.75361 E-03$ $1.90293 E-02 \quad 2.44307 E-04$ $5.13907 E-04 \quad 1.15232 E-06$ 3.05175E-05 $2.09072 E-06$ 2.97419E-05 $2.66579 E-06$ 5. $59662 E-05 \quad 3.87538 E-06$ $0.65469 E-05 \quad 6.00227 E-06$ 1.07121E-04 9.66190 E-06 $1.81720 E-04 \quad 1.63904 E-05$ $5.37141 E-04$ 2.52709E-05 $5.37141 E-04 \quad 4.84480 E-05$ $1.95368 \mathrm{E}-03$ 1.076211E-04 $3.44885 E-03 \quad 3.11067 E-04$ $6.01760 E-03 \quad 5.42760 E-04$ $\begin{array}{ll}9.92121 E-03 & 8.94861 E-04 \\ 4.08663 E-02 & 3.686 C 3 E-03\end{array}$ 


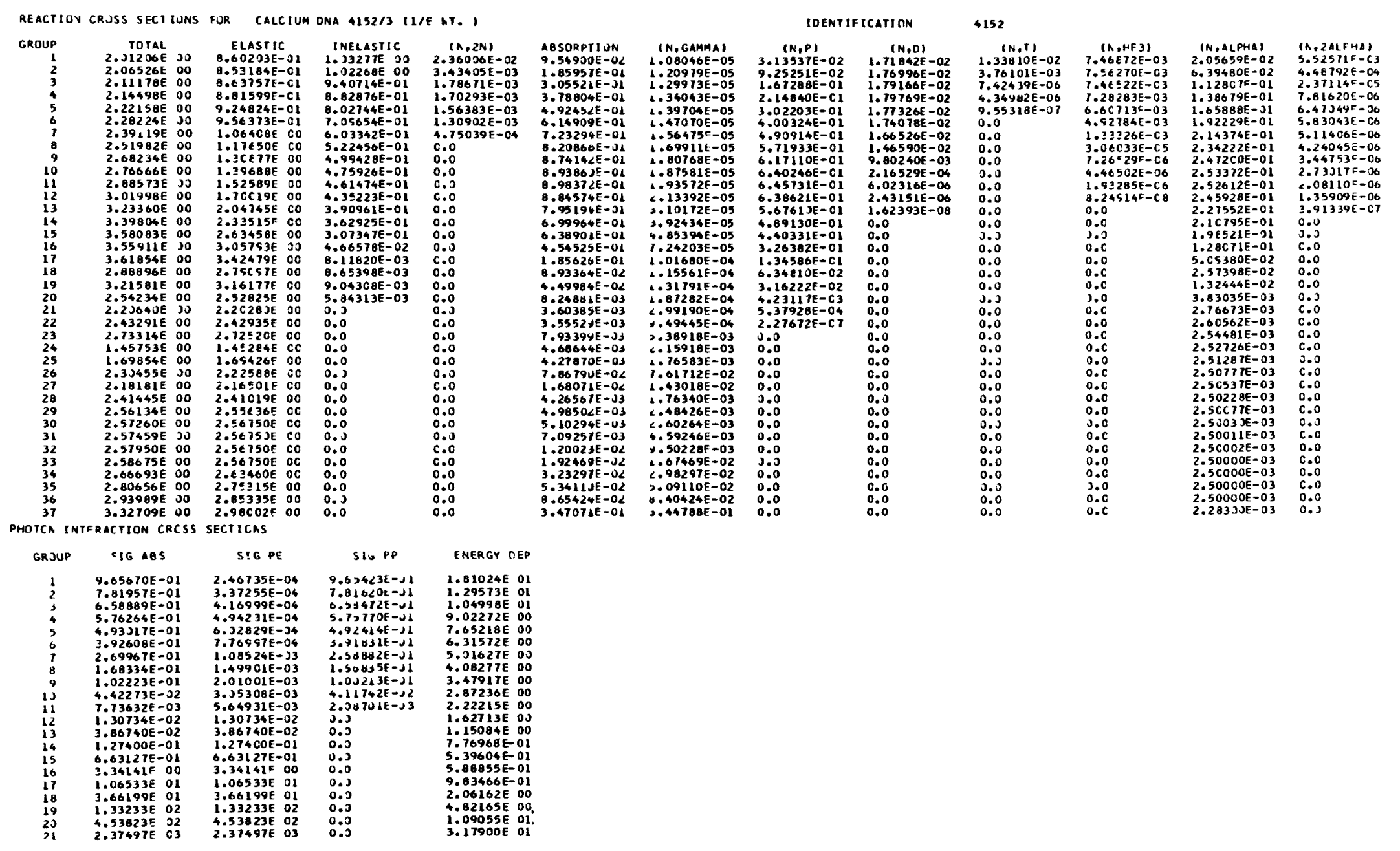




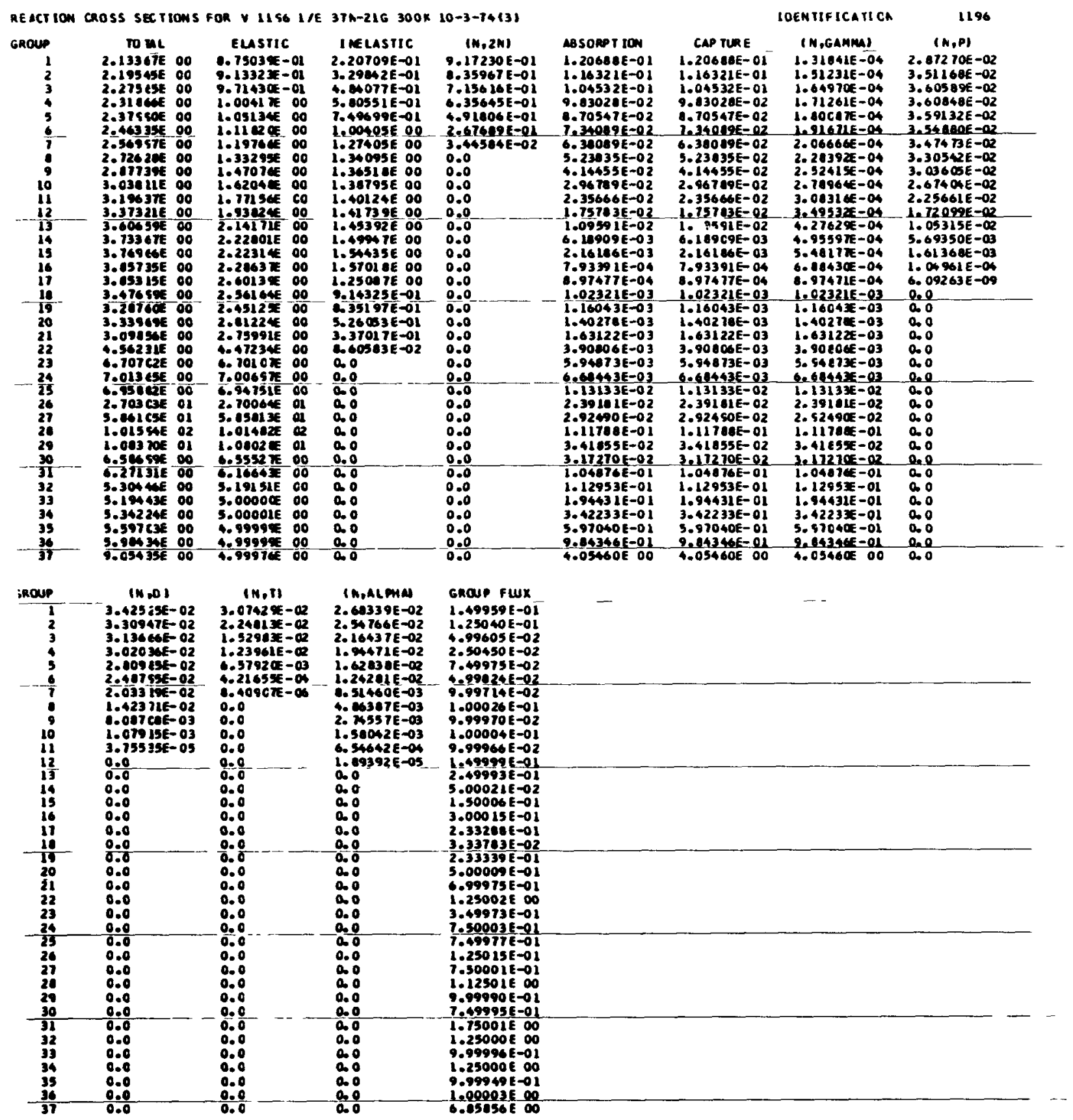


PHOTON INTERACTION CROSS SECTIONS FOR V

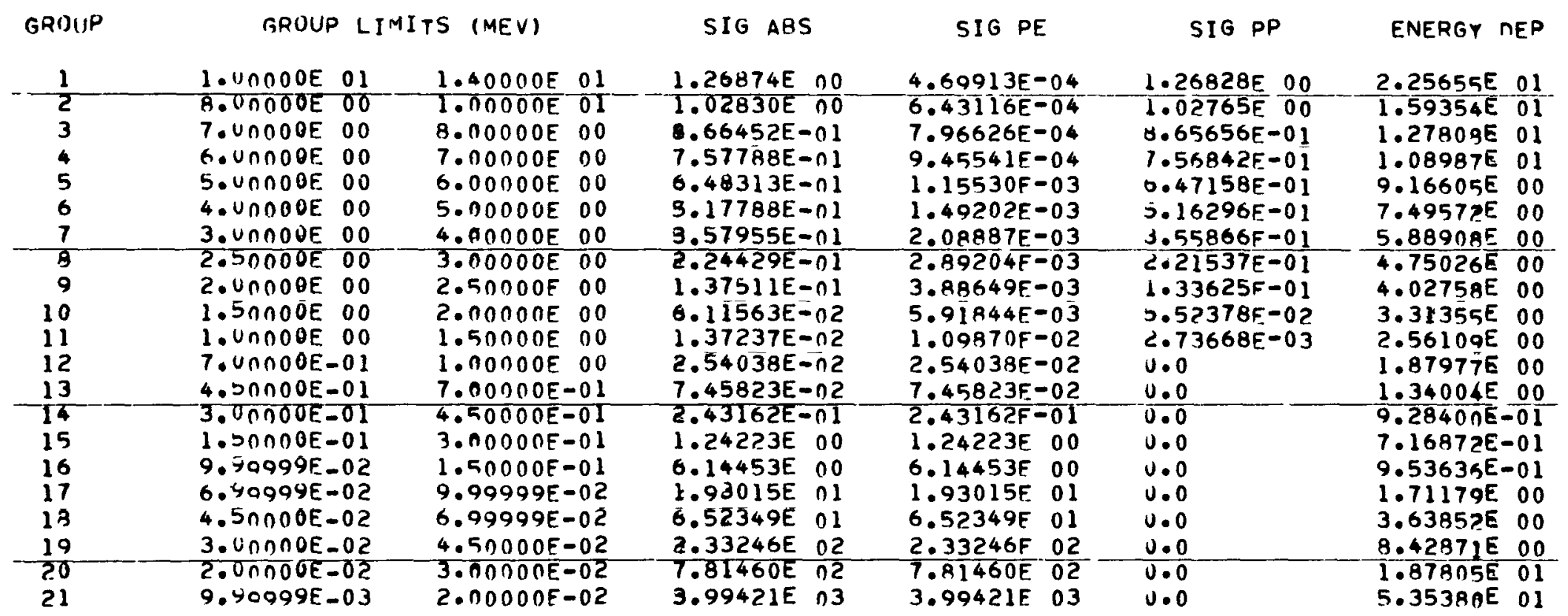




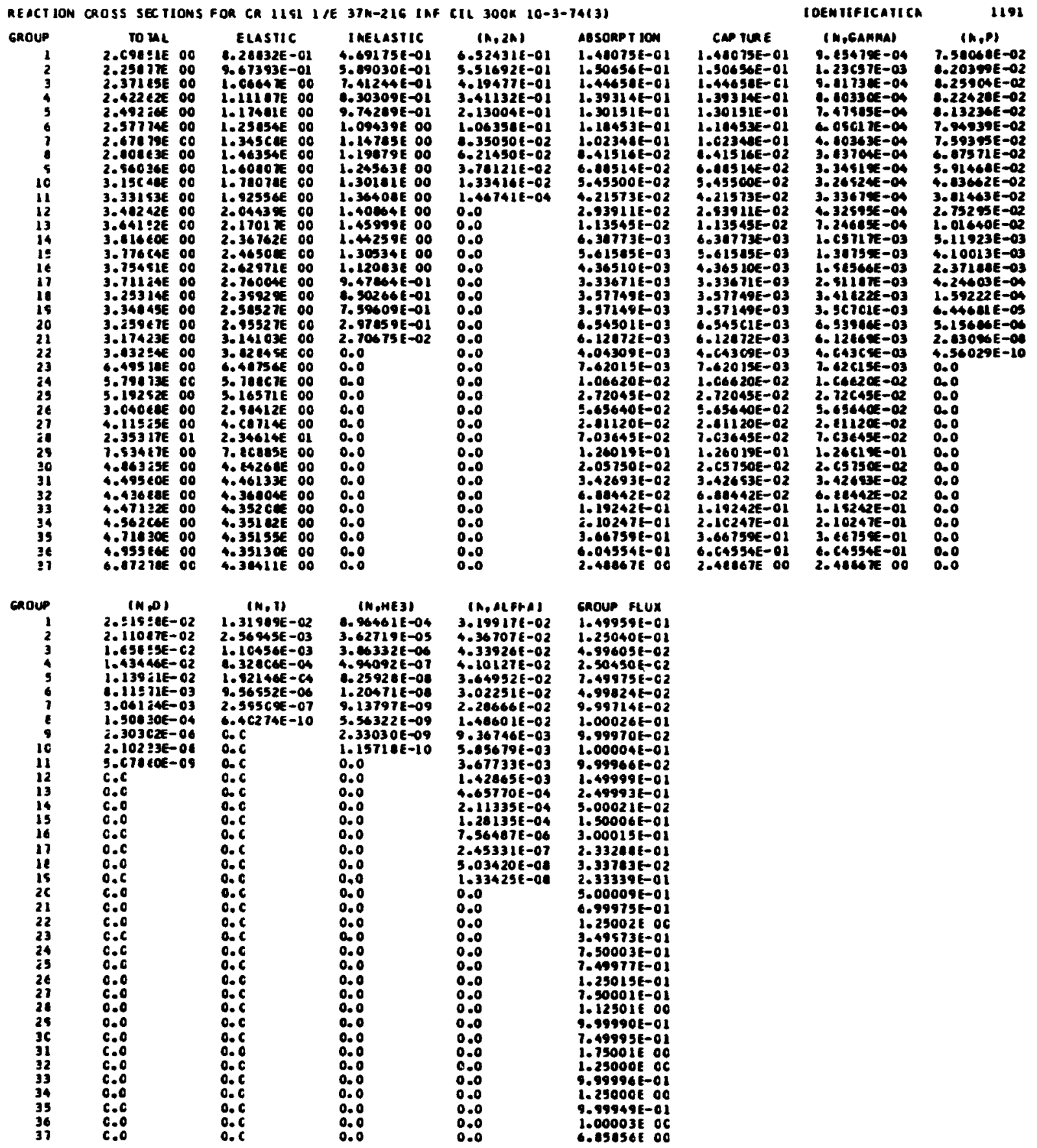


PHOTON INTERACTION CROSS SECTIONS FOR Cr

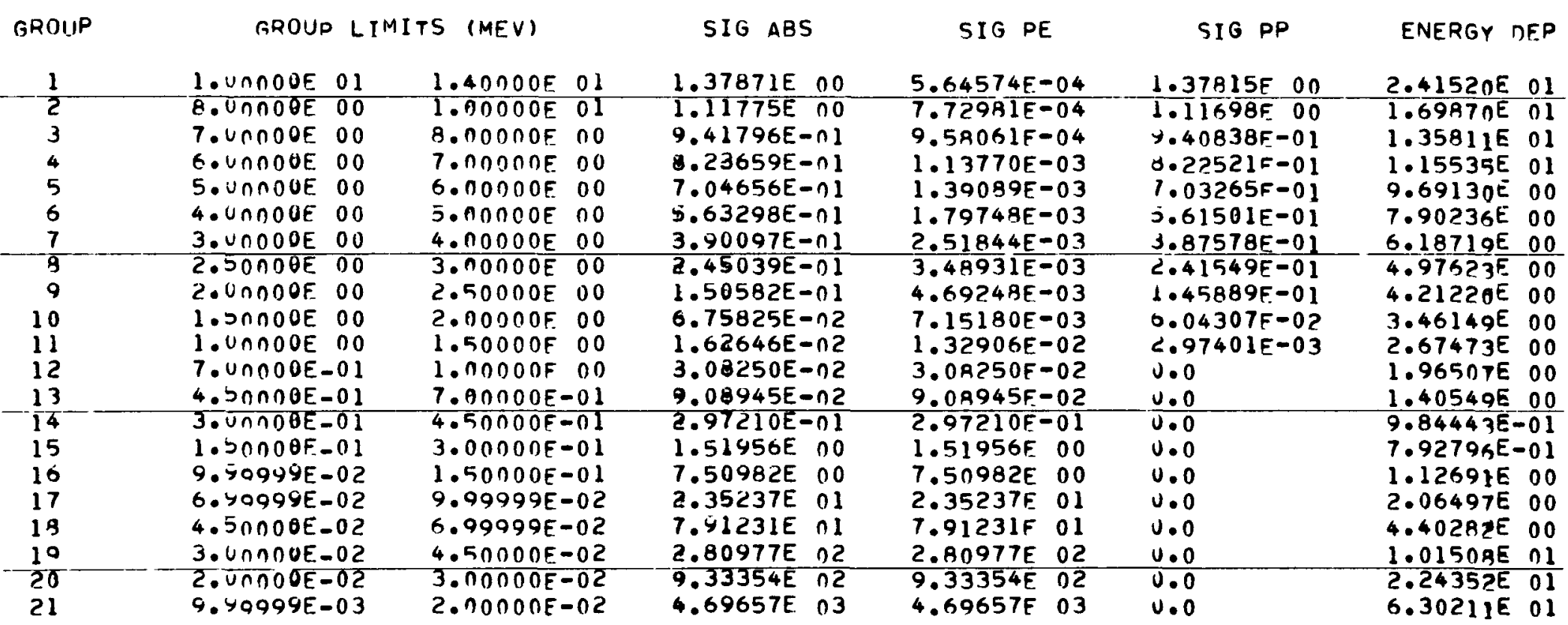




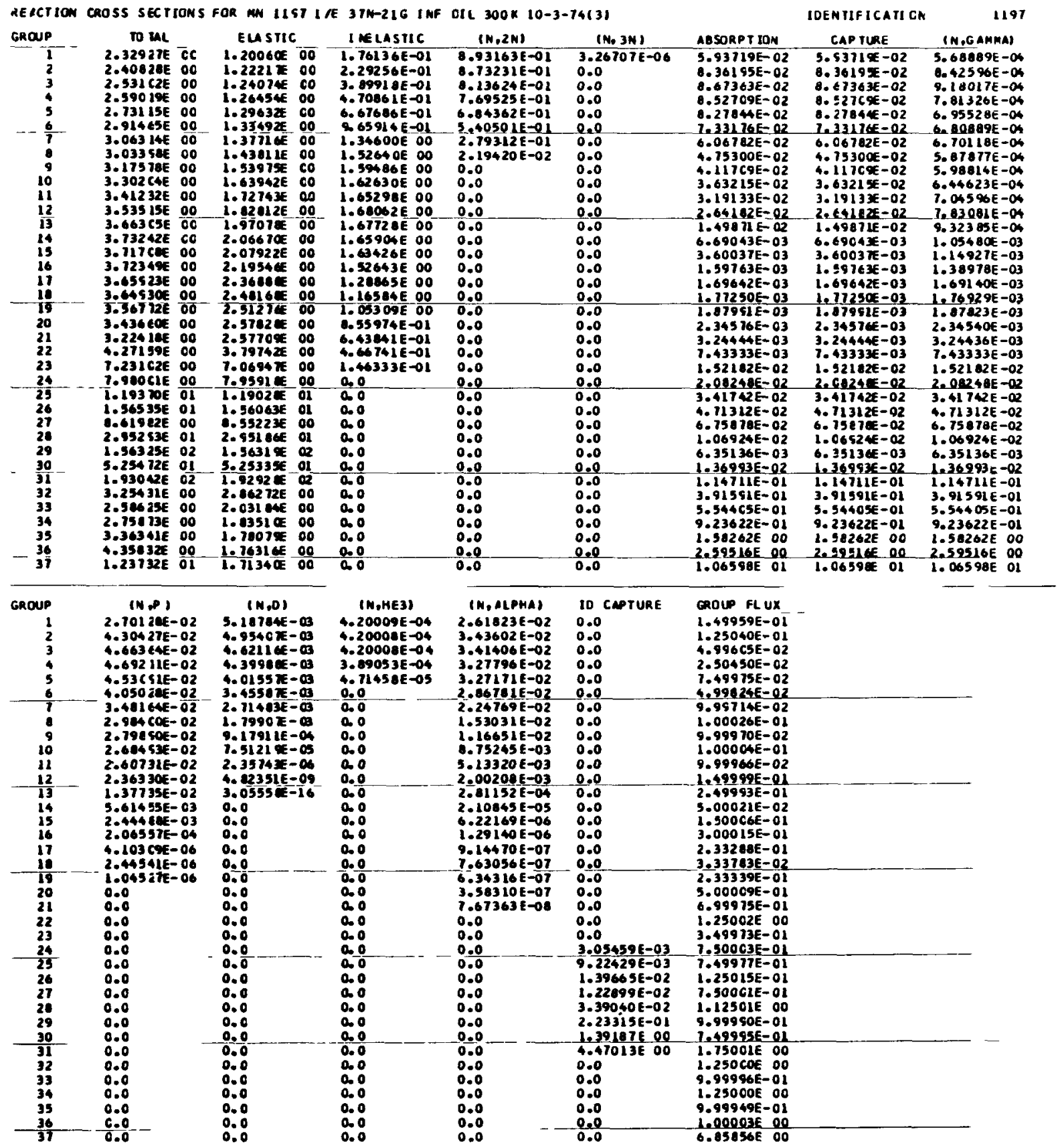


PHOTON INTERACTION CROSS SECTIONS FOR Mn

\begin{tabular}{|c|c|c|c|c|c|c|c|}
\hline GROUP & GROUP L & (MEV) & SIG ABS & SIG PE & SIG PP & ENERGY & NEP \\
\hline 1 & $1.60000 E$ & $1.40000 E \quad 01$ & $1.49317 \mathrm{E}$ & $6.92375 F-04$ & $1.49248 E \quad 00$ & $2.57881 E$ & 01 \\
\hline 2 & $8.0 \cap 000 E$ & $1.00000 E 01$ & $1.21086 E$ & $9.48371 E=04$ & $1.20991 \mathrm{~F} 00$ & $1.80679 E$ & 01 \\
\hline 3 & $7 . U \cap 000 E$ & $8.00000 F \quad 00$ & $1.02022 E \quad 00$ & $1.17616 E-03$ & $1.01905 E 00$ & $1.44015 \mathrm{E}$ & 01 \\
\hline 4 & 6. UกกOOE & $7.00000 E \quad 00$ & $0.92240 E-01$ & $1.39736 E-03$ & $8.90843 F-01$ & I. $22233 \mathrm{E}$ & $0 i$ \\
\hline 5 & $5 . U \cap \cap 0 \theta \bar{E}$ & $6.00000 E 00$ & $7.63324 E-01$ & $1.70926 \mathrm{E}-03$ & $7.61615 E-01$ & $1.02271 \mathrm{E}$ & 01 \\
\hline 6 & 4. UกกOOE & $5.00000 E \quad 00$ & $0.10731 E-01$ & $2.21028 E-03$ & $0.08521 E-01$ & $8.31583 E$ & 00 \\
\hline 7 & 3. UNOOOE & $4.10000 E \quad 00$ & $4.23732 E-01$ & $3.09919 E-03$ & $4.20633 E-01$ & $6.4891 \mathrm{AE}$ & 00 \\
\hline 8 & 2.500008 & $3.70000 E 00$ & $2.66764 E-01$ & $4.29719 E-03$ & $2.62467 F=01$ & $5.20435 E$ & 00 \\
\hline 9 & $2.00000 E$ & $2.50000 E \quad 00$ & $1.64520 E-01$ & $5.78306 E-03$ & $1.58737 E-01$ & $4.39819 E$ & 00 \\
\hline 10 & $1.20000 E \quad 00$ & $2.10000 E$ กO & $7.47007 E-02$ & $8.82135 E-03$ & $0.59794 F-02$ & 3.610386 & 00 \\
\hline 11 & I. UNOOOE OO & $1.50000 E$ OO & $1.96319 E-02$ & $1.64101 E-02$ & $3.22182 F=03$ & $2.78936 E$ & 00 \\
\hline 12 & 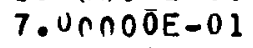 & $1.00000 E \quad 00$ & $3.7 \overline{8} 653 E-02$ & $3.78653 E-02$ & 0.0 & $2.05171 E$ & 00 \\
\hline 13 & 4. $\supset \cap \cap 00 E-01$ & 7.00000E -01 & $1.10425 E-01$ & $1.10425 \mathrm{~F}-01$ & 0.0 & 1.472736 & 00 \\
\hline 14 & 3. UחกOOE-01 & $4.50000 E=01$ & $3.57497 E-01$ & $3.57497 E-01$ & 0.0 & $1.0427 \mathrm{AE}$ & 00 \\
\hline 15 & $1.50000 E=01$ & $3.00000 E-01$ & $1.80716 E \quad 00$ & $1.80716 E \quad 00$ & U. 0 & $8.71027 E$ & -01 \\
\hline 16 & $9.99999 E-02$ & $1.50000 E-01$ & $8.85626 E \quad 00$ & $8.85626 \mathrm{E} \quad 00$ & 0.0 & $1.2981 \mathrm{DE}$ & 00 \\
\hline 17 & 6. SС999E-02 & $9.99999 E-0 ?$ & $2.75978 E$ OI & $2.7597 A E \quad 01$ & 0.0 & $2.40606 \mathrm{E}$ & 00 \\
\hline 18 & $4.50000 E-02$ & $6.99999 E-02$ & $9.23297 E$ & $9.23297 E$ OI & $0 \cdot 0$ & $5.13010 E$ & 00 \\
\hline 19 & $3.0000 \theta E-02$ & $4.50000 E-02$ & $3.26320 \mathrm{E} \quad 02$ & $3.26320 \mathrm{~F} \quad 02$ & 0.0 & 1.178655 & 01 \\
\hline $\begin{array}{l}20 \\
21\end{array}$ & $\begin{array}{l}2.00000 E-02 \\
9.49999 E-03\end{array}$ & $\begin{array}{l}3.000 O 0 E-02 \\
2.00 \cap 00 E-02\end{array}$ & $\begin{array}{l}\text { I.07917E } n 3 \\
\$ .39370 \mathrm{E} \text { n3 }\end{array}$ & $\begin{array}{ll}.07917 \mathrm{E} & 03 \\
5.39370 \mathrm{E} & 03\end{array}$ & $\begin{array}{l}0.0 \\
0.0\end{array}$ & $\begin{array}{l}2.594206 \\
7.240456\end{array}$ & 01 \\
\hline
\end{tabular}




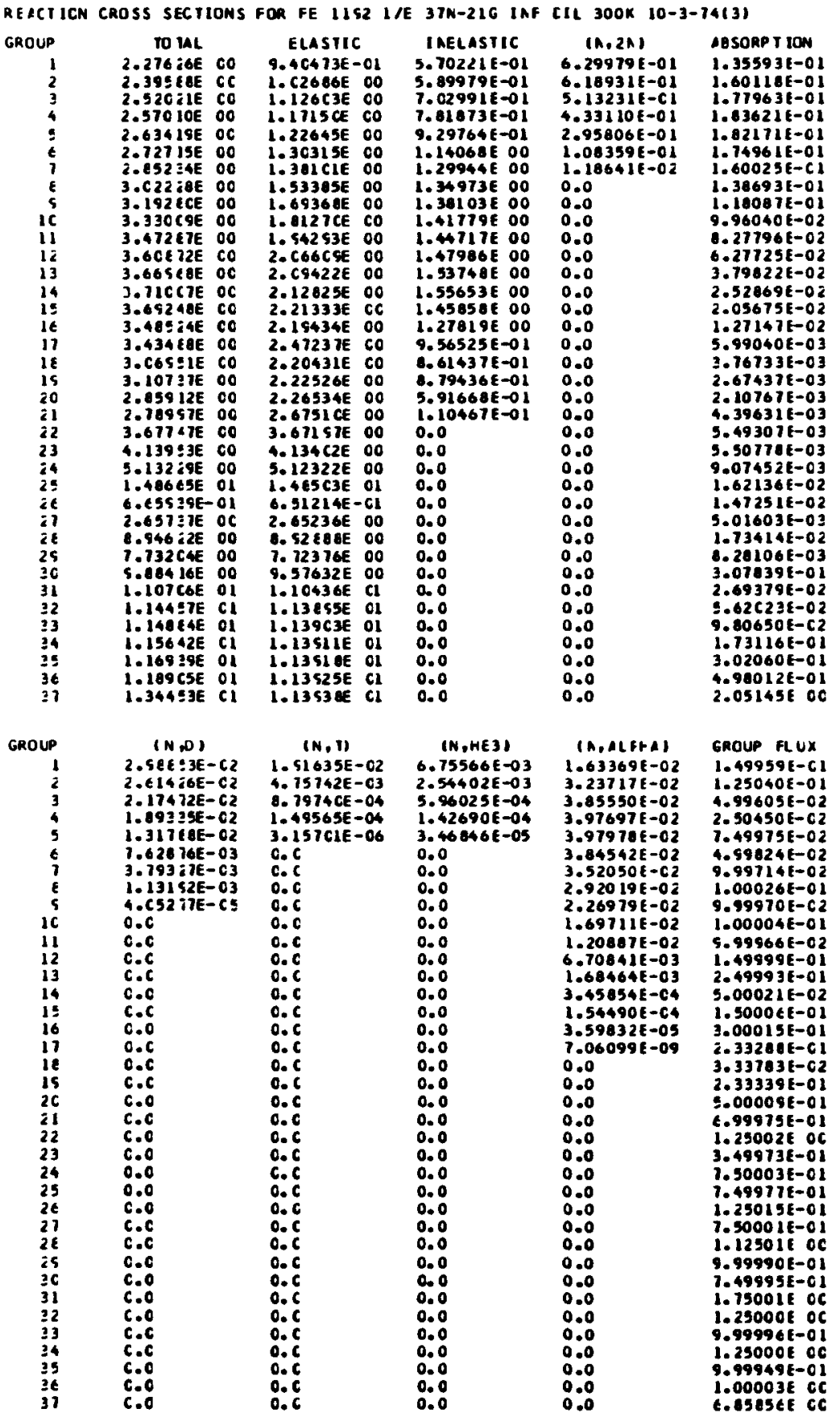

IDEN IIFICATICH

1192 CAP TURE

$1.35553 E-01$
$1.60118 E-01$ 1.60118E-01 $1.83621 E-01$ 1.82171E-01 1.74961E-01 $1.60025 E-c 1$ 1.38653E-OI 1.18087E-0I S. $56 C 40 E-02$ $0.27786-02$ $6.27725 E-02$ $3.79 \in 22 E-02$ 2.52069E-02 $2.05675 E-02$ I.ZT147E-02 5.95CAOE-03 $3.76733 E-03$ $2.67437 E-03$ $2.10767 \mathrm{E}-03$ $4.39631 E-03$ 5.493 CTE-03 $5.50778 E-03$ S.C7452E-03 $1.62136 E-02$ $1.47251 E-C 2$ 5.016C3E-03 i. $33414 E-02$ $0.201005-03$ 3.C7939E-OI $2.69379 E-02$ 0.62 C23E-0Z $9.00650 E-02$ $1.73116 E-01$ 3.C2060E-O $4.58012 E-01$ 2.05145E CO

(M.gAMMA) $(A, P)$

1. 3327 CE-04 6.32785E-02 1. 51 ECgE-04 9.41105E-02 2. 1 C324E-04 1.24416E-01 $2.16285 E-04 \quad 1.28938 E-01$ 2. $286 C O E-06 \quad 1.28650 E-01$ 2.2E6COE-06 1.28650E-01 2.EC257E-04 1.08099E-01 2. iefCOE-04 9.50699E-02 3. C503AE $-04 \quad 8.23279 E-02$ 3.41543E-04 $7.03495 E-02$ $\begin{array}{ll}3.41543 E-04 & 7.03495 E-02 \\ 3.86196 E-04 & 5.56779 E-02\end{array}$ $3.56779 E-02$
$4.45178 E-04$
$3.58283 E-02$ 5. 1 SI $3 E-04$ $2.43925 E-02$

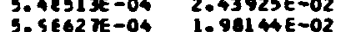
7. $53690 E-04$ 1.19251E -02 7. $3650 E-04$ 1.19251E-02 9. ISCCCE-04 S. OI134E-03 1.10CCZE $-03 \quad 2.66730 E-03$ 1.2eE29E-03 1.38609E-03 1. SSCC $3 E-03$ 1.52245E-04 S. $453(3 E-03 \quad 3.70399 E-00$ 5. 4 c 13 5. $5 C 71<-03$ 1. $2136 \mathrm{E}-02$ I. $47251 E-02$ CI $6 C 3 E-03$ 1. $73414 E-02$ Q. 2 el CGE -03 3. $27639 E-01$ 2. $6937 \%-02$ - 2 2C23E-02 S. 2065 OE -02 i. $73116 E-01$ 3. C2CSCE-OI 3. 2. csisse oC

0.

0.0

0.0

0.0
0.0
0.0
0.0

0.0

0.0

0.0

0.0

0.0

0.0

0.0 0.0

0.6

c.sseseE CC 
PHOTON INTERACTION CROSS SECTIONS FOR Fe

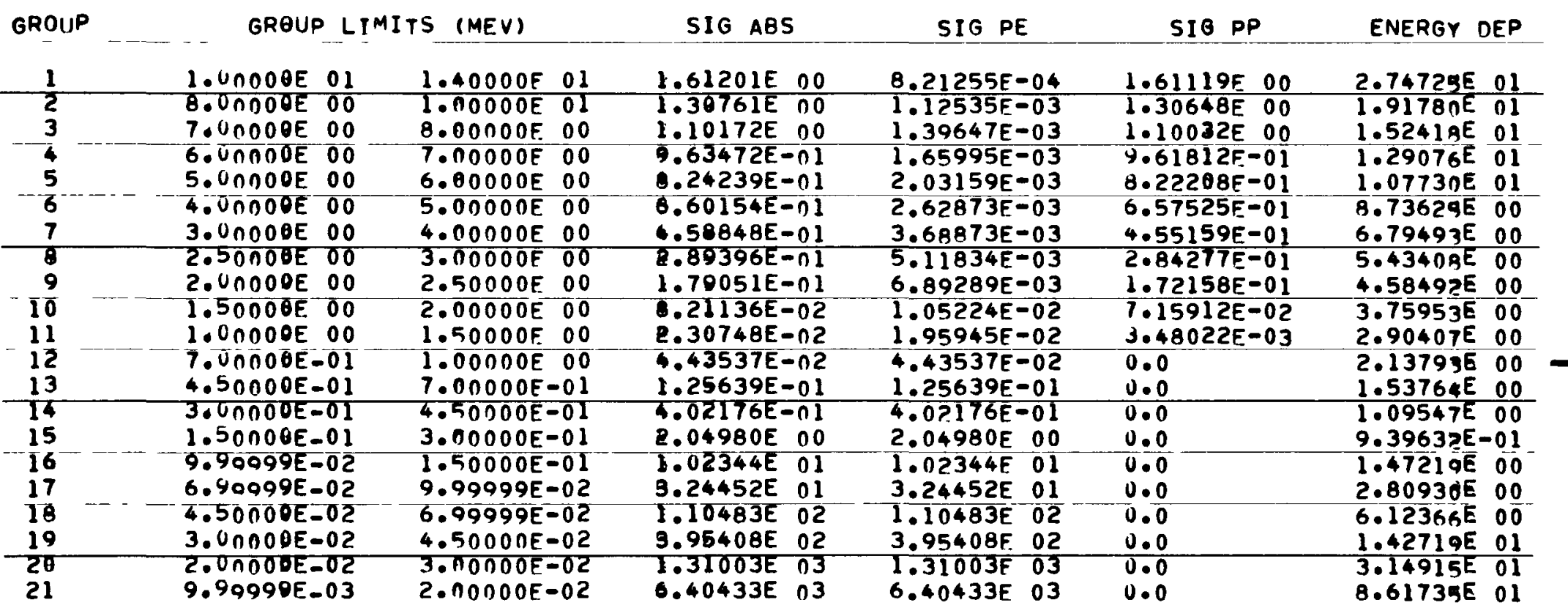




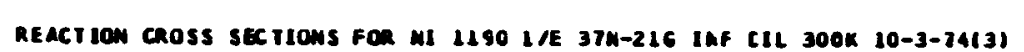
coup
1
2
3
3
5
5
5
11
11
12
13
15
16
17
11
15
20
21
22
23
24
29
21
21
21
29
30
31
32
33
34
35
36
31

ro $\mathrm{UL}$

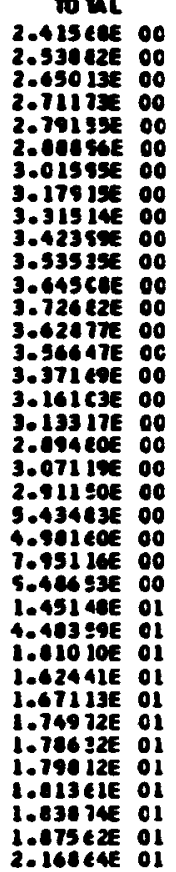
elastic

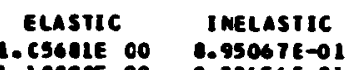
(N.2N)

acsoner 1 ion

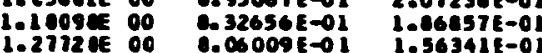
..32712E OO C.05013E-O $1.39407 E$ OO $0.01332 E-01$

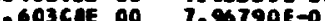
$24157 E$ 00 $03682 E-01$ 1.4006C 00 O.06344E-01 2. 9ง92Le 176735 OO $1.12519 E$ OO 2. C2794E 00 1. 96910 E 00 $1.24624 E 00$ $30134 E 00$ T.64497E 01 L 0.0 CASOLE 0O 2.90364e 00 0.0 $3.42565 E$.0 0.0 7. $93311 \mathrm{LE} 00 \mathrm{00}$ 2. 4504te o0 0.0 4.47370 of ol 0.0 $1.62004 \mathrm{f}$ ol 0.0 1. 34463E of 0.0 1. 77625E of 0.0 1. 79057E of 0.0 1. TaAjoe ol 0.0 1. Je654E ol 0.0
IDEM HIF ICATI CM

\begin{tabular}{|c|c|c|}
\hline 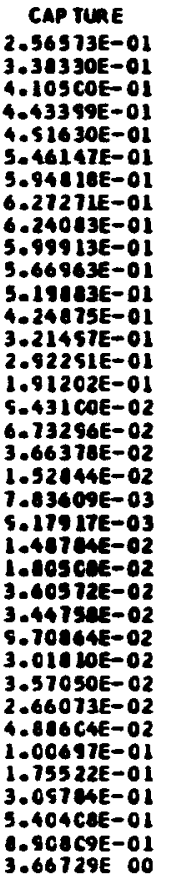 & 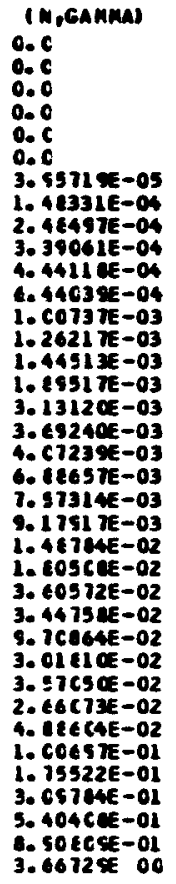 & 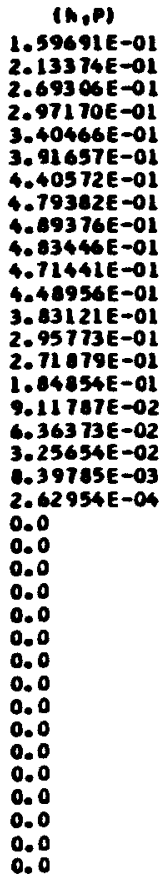 \\
\hline
\end{tabular}
.249ESE-CI 1.2504CE-CI .415 S4E-01 4. \$\$6CSE-02 1.462 29E-01 $2.5 C 45 \mathrm{CE}-\mathrm{CZ}_{2}$ 1.511<4E-01 7.45575E-C2 1.542 CBE- OL S. \$5714E-0 1.C0026E-01 1.34458E-Cl S. $59970 E-02$ 1.16120E-C1 1.CCOCAE-01

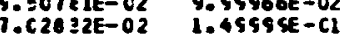
$4 . C 74 E 2 E-02$ 2.455\$3E-CI $2.44610 E-02$ 5. $00021 E-02$ $1.096 E 8 E-02 \quad 1.5 C 0 C 6 E-01$ (1) $0 . c$ 0.0 $6.73296 \mathrm{E}-02$ $1.521446-0$ 1.03609E-03 $9.179175-03$ 1. $605061-02$ $3.447504-02$

$9.70464 E-02$

$3.01010 \mathrm{-02}$

$3.570501-02$

4.08604 t- 02

$1.006971-01$
$1.75522 t-021$

3.09784E-O $9.9069 \mathrm{E}-01$

$3.9689929-01$

3.6672\% 00
3. CCCL SE-01 $3.33703 E-C 2$
3.3333 SE -01 5. COCCYE - 01 $6.99975 E-01$
$1.25002 E-00$ $1.25002 E$
$3.49973 E-01$ 7. SCCCBE-01 7.4957TE-01 $1.25015 E-01$
$7.50 C C 1 E-01$ 7. SOCCLE-01 \$. $599906-01$ $7.45595 E-02$ $1.75001 E$ OD $1.25 C C E$
$\$ .55996 E-01$ 1.25000E 00 $1.0 \mathrm{CCOOBE} 00$
$6.65056 \mathrm{CO}$ 
PHOTON INTERACTION CROSS SECTIONS FOR Ni

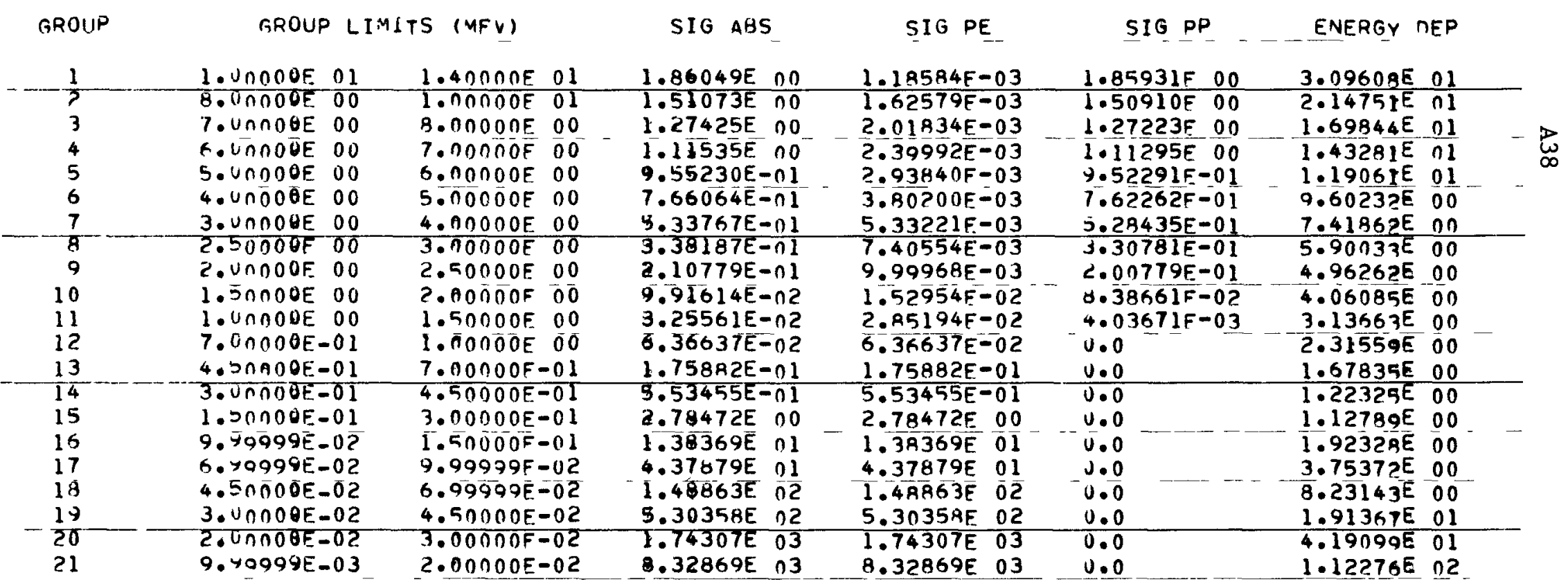




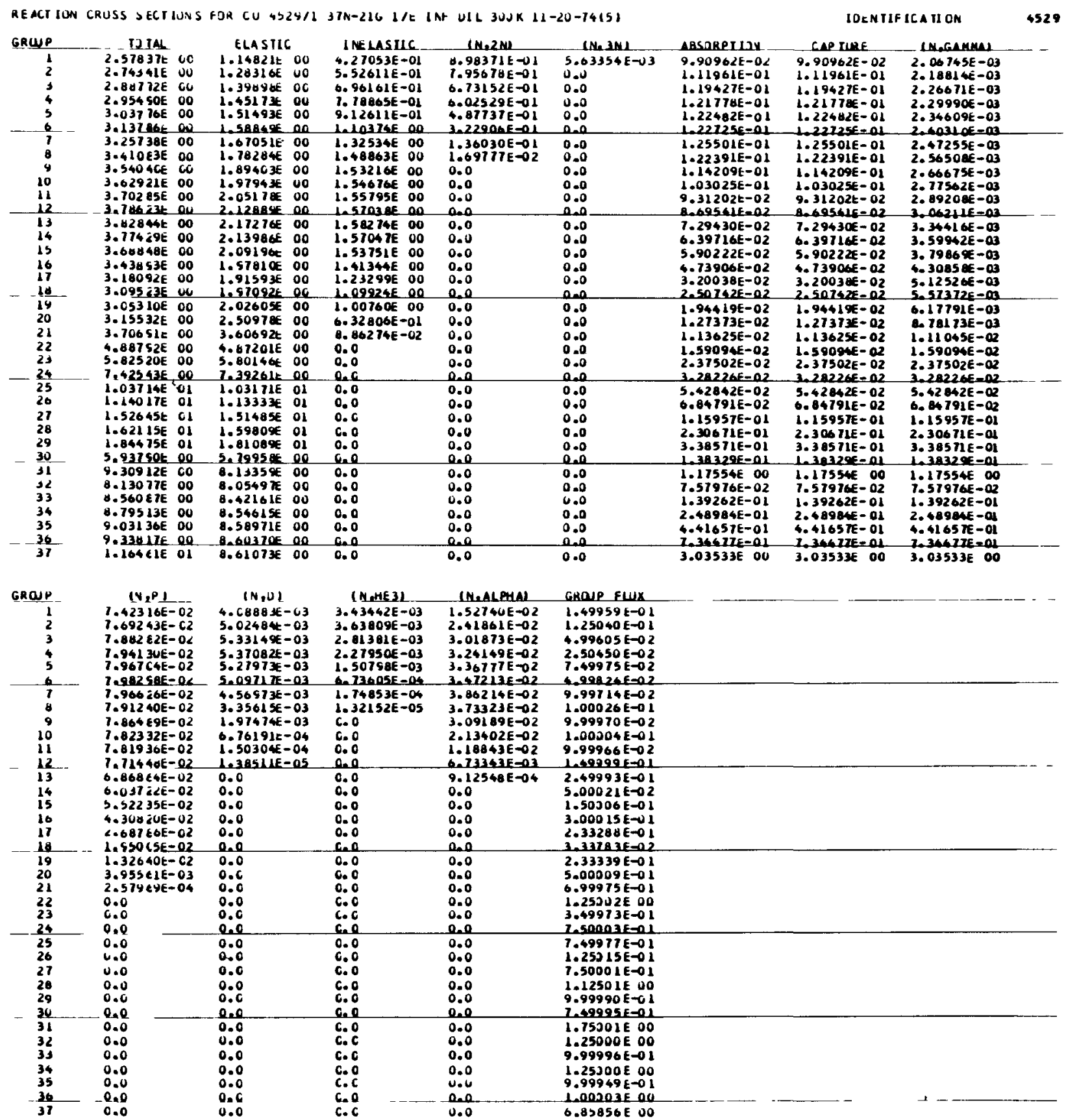


PHOTON INTERACTION CROSS SECTIONS FOR Cu

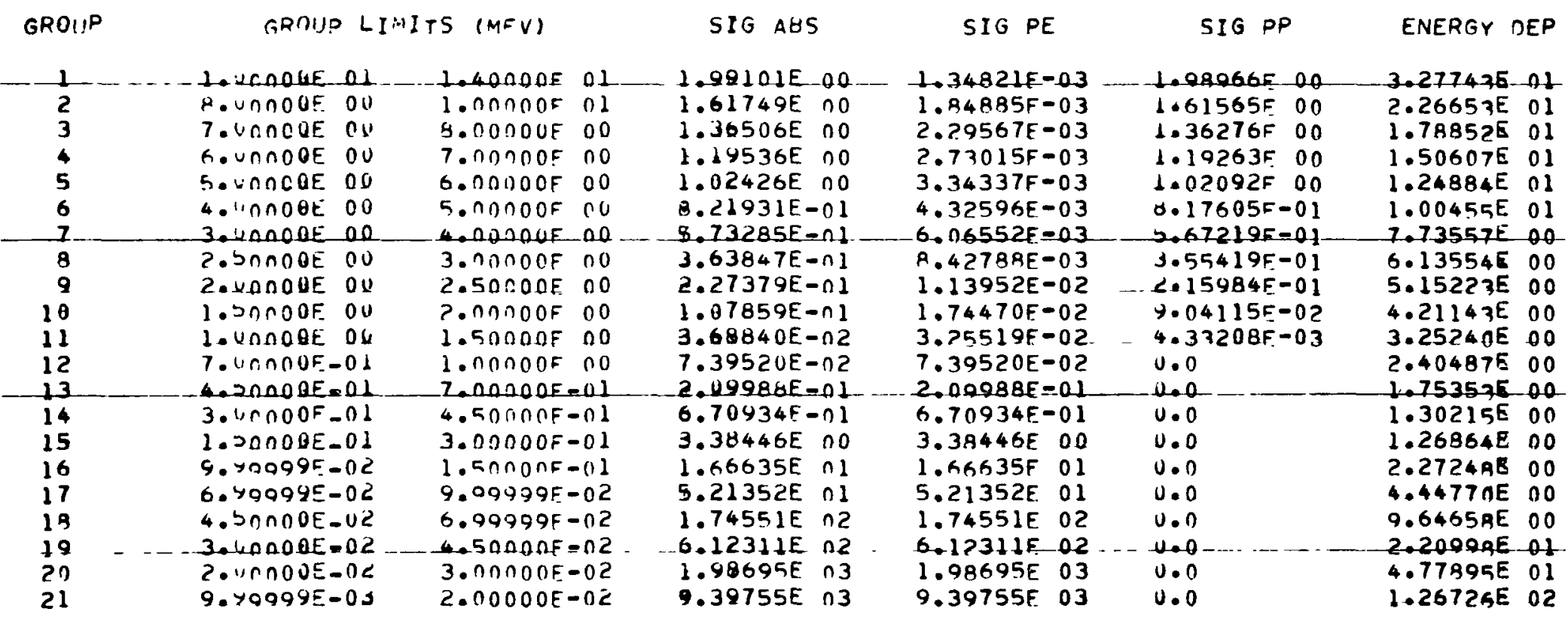




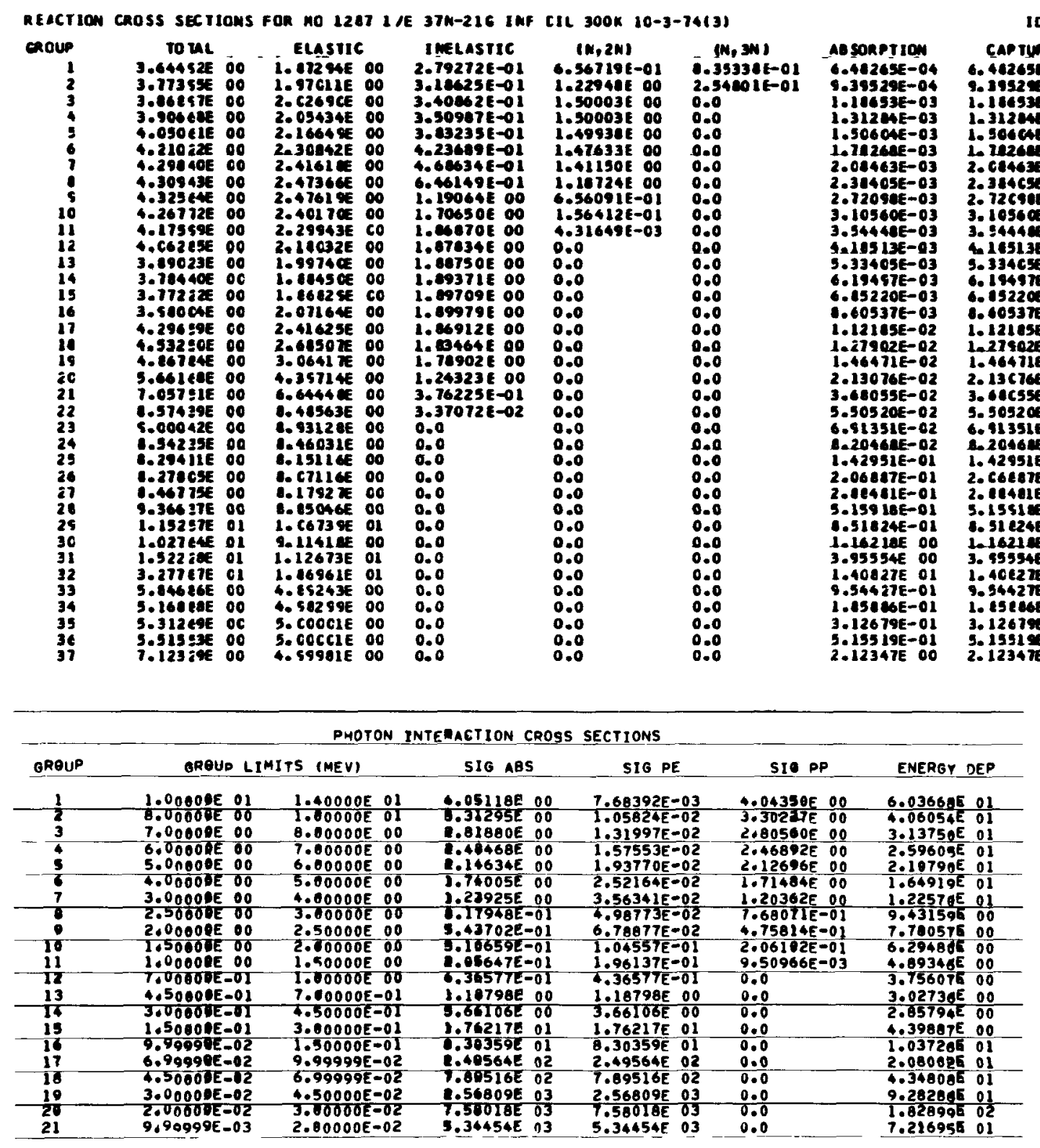

den HiF C CATI Ca

(N,Ganma) EROLP Flux 6.40265E-04 1.455:SE-C1 1.CS3E-03 $1.10653 E-03$ 4.99ECSE\begin{tabular}{l}
$1.30604 E-03 \quad 1.49975 E-C_{2}$ \\
\hline
\end{tabular}

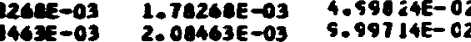

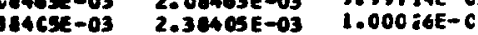
2.72090E-03 S.599 70E-C2 3.10560E-03 1.COC C4E-C $4.10513 E-03$ 1.49539E- 01 5. $33405 E-03$ 2.4953EC. $0.0537 E-03 \quad 3.00015 E-0$ $1.12105 E-02$ 2.3328EE-C $1.27002 E-02 \quad 3.337 \in 3 E-C 2$ $13076 \mathrm{E}-02$ s.00c 3.60055E-02 C.59975E-CI 5.50520E-02 1.230C2E OC $.020460 E-02$ 7.500C3E-01 $1.25015 E-0$

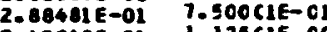
3.15910E-01 1..12दcte $1.16210 E$ 0O $7.499555-01$ $1.40027 \mathrm{E}$ ol $1.250 \mathrm{icce}$ OC 9.5 $5427276-0125.5599565-01$ 3.12679E-0I 9. $59949 E-C$

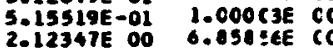




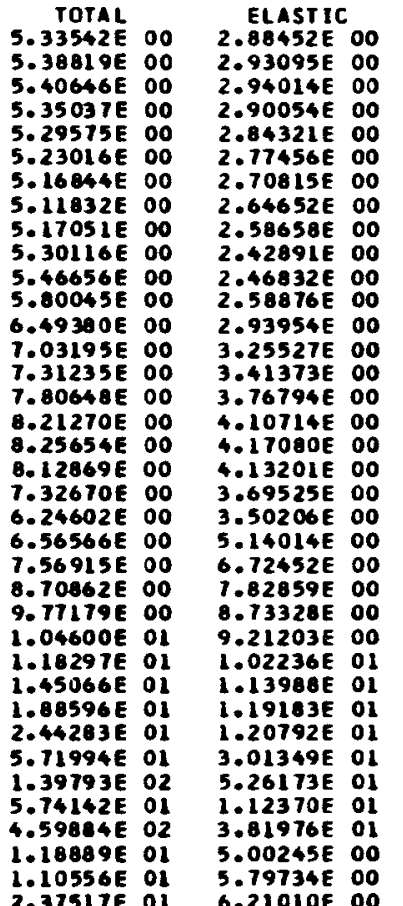

INELASTIC (N,2NI $2.30003 E-01$ $2.30002 E-01$ $2.36691 E-0$ $3.61347 \mathrm{E}-01$ $6.35179 E-01$ 2.26647E 00 $2.93757 E$ OO $3.54302 E 00$ $3.76359 E$ 0O $3.80441 E$ OO $4.07106 E 00$ $4.04103 E 00$ $3.94197 E 00$ 3.53578E 00 1.17577 0O $5.66968 E-01$ $5.05173 \mathrm{EE}-01$ $3.16601 E-01$ $3.16601 E-01$
$2.06091 E-01$ 2.23796 E-02 0.0
0.0 0.0 0.0
0.0 0.0
0.0 0.0 PHOTO

SIG ABS

SIG PE

.12757 OI $9.38635 E$ OD $8.17623 E$
$7.34978 E$ 7.34978E OO $5.48288 E$ OO $4.21052 E 00$ $3.14270 E O O$ $2.48402 E 00$ $2.07073 E 00$ $2.48966 E$ OO $5.18338 E$ OO 3.63514E ol $1.60059 E$ O2 $6.96063 E$ O2 1.37883E 03 1.07195E O4

$$
\begin{aligned}
& 9 . \\
& 1.0 \\
& 2 . \\
& 2.2 \\
& 2.2 \\
& 2.2 \\
& 2.0 \\
& 1.02 \\
& 1.05 \\
& 5.9 \\
& 0.0 \\
& 0.0 \\
& 0.0 \\
& 0.0 \\
& 0.0 \\
& 0.0 \\
& 0.0 \\
& 0.0 \\
& 0.0 \\
& 0.0 \\
& 0.0 \\
& 0.0 \\
& 0.0 \\
& 0.0 \\
& 0.0 \\
& 0.0 \\
& 0.0 \\
& 0.0 \\
& 0.0 \\
& 0.0 \\
& 0.0 \\
& 0.0 \\
& 0.0 \\
& 0.0 \\
& 0.0
\end{aligned}
$$
$\begin{array}{cc}\text { (N,3N) } & \text { ABSORPTION } \\ 1.299306 \mathrm{~N} \text { OO } & 1.09378 E-02 \\ 4.94430 E-01 & 1.04143 E-02\end{array}$ $.04143 E-02$
$9.05433 E-03$ 8.22208E-03 $7.16743 E-03$ $6.02612 E-03$ $6.22959 E-03$
$7.02285 E-03$ $7.75000 E-03$ $0.41064 E-03$ $9.00811 E-03$ $9.672377 E-02$
1.12372
$1.30852 E-02$ $1.42113 E-02$ $3.45093 E-02$
$4.47107 E-02$ $5.47218 E-02$ $9.56729 E-02$ $2.49750 E-01$ $2.77664 E-01$ $3.74861 E-01$ $9.31386 E-01$ $1.40002 E$ OO 6.9414 $6.94141 E$
$1.23491 E$ $2.70645 E$ O1 $8.71759 E$ O1
4.61771 01 $4.61771 E$ O1
$4.21686 E$ O2 $6.88649 E 00$ $5.25827 E$ OO (N,GAMMA)
$3.43938 E-03$
$4.49840)-03$ $\begin{array}{ll}3.43938 E-03 & 7.49840 E-03 \\ 4.02758 E-03 & 6.38674 E-03\end{array}$ $4.36270 E-03 \quad 4.69162 E-03$ $\begin{array}{ll}4.49656 E-03 & 3.72552 E-03 \\ 4.6671 E-03 & 2.4961 E-03\end{array}$

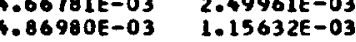
6.32376E-03 9.06226E-04 $7.06854 E-03$
$7.063428368 E-04$ $7.82578 E-03 \quad 5.84869 E-04$ $8.51064 E-03$
$9.27029 E-03$
$1.0974696-04$ $1.006930^{-02}$. $1.09693 E-02 \quad 2.68381 E-04$ $1.40370 E-02 \quad 1.9340 E-04$ $\begin{array}{ll}1.40339 E-02 & 1.74336 E-04 \\ 1.983747 E-04 & \end{array}$ $\begin{array}{ll}3.44061 E-02 & 1.03235 E-04 \\ 4.46217 E-02 & 8.90390 E-05\end{array}$ S. $56449 E-02$ $7.69449 E-05$ $1.53172 E-01 \quad 2.42713 E-05$ $2.49746 E-01 \quad 4.37805 E-06$ 2.77664E-01 0.0 6.540100-01 0.0 $9.31386 E-010.0$ 1.40002E 000.0

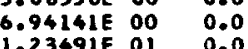
2.70645 O1 0.0 8.71759E 010.0 $\begin{array}{lll}4.61711 E & 01 & 0.0 \\ 4.21686 E & 02 & 0.0\end{array}$ $\begin{array}{lll}5.25827 E & 00 & 0.0 \\ 1.75416 E \text { o1 } & 0.0\end{array}$ (N, 3NI
N $1.04143 \mathrm{E}-02$ $6.88649 E 00 \quad 0.0$ 8. $46162 E-02$
$1.17426 E-01$ 1.47974E-01 1. $78091 E-01$ $2.21162 E-01$ $2.91919 E-01$ $5.98577 E-01$ $8.25541 E-01$ $1.28875 E$. 2..45450e 00 1.27109601 $3.63514 E$ O1 $1.60859 E 02$ $6.96063 E$
1.914717 $1.37883 E$ 03

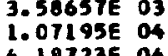

S1G PP $1.119110^{01}$ $9026825 E 00$ $.17169 E 0 O$ $6.28732 E 00$ $3.78999 E 0 O$ 2.54412E 00 $1.65848 E$
$7.0987 E-01$ .51612E-02 0.0

0.0

0.0

0.0
ENERGY DEP $1.51736 E 02$ T.59150e ol $6.19075 E$ ol .92953E ol $2.68044 E$ OI .99033E ol .31806 O1 . $10891 E$ ol i.11503E ol $.58296 E$ OI
$3.40173 E$ ol $8.45460 E$ O1
$1.58722 E$ O2 $7.95510 E$ O1 $2.58427 E 02$ 


\begin{tabular}{|c|c|c|c|c|c|c|c|c|c|c|c|}
\hline REACTION & CROSS SECTION & $N-182$ & DNA $4582 / 211 /$ & E WT. I & & & IDENT I & ICATION & 4582 & & \\
\hline $\begin{array}{r}\text { GRoup } \\
1 \\
2 \\
3 \\
4 \\
5 \\
6 \\
7 \\
8 \\
9 \\
10 \\
11 \\
12 \\
13 \\
14 \\
15 \\
16 \\
17 \\
18 \\
19 \\
20 \\
21 \\
22 \\
23 \\
24 \\
25 \\
26 \\
27 \\
28 \\
29 \\
30 \\
31 \\
32 \\
33 \\
34 \\
35 \\
36 \\
37\end{array}$ & 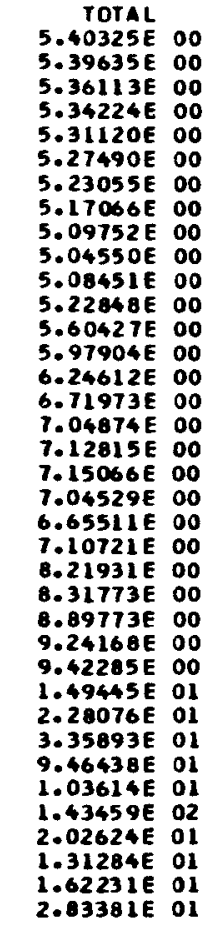 & 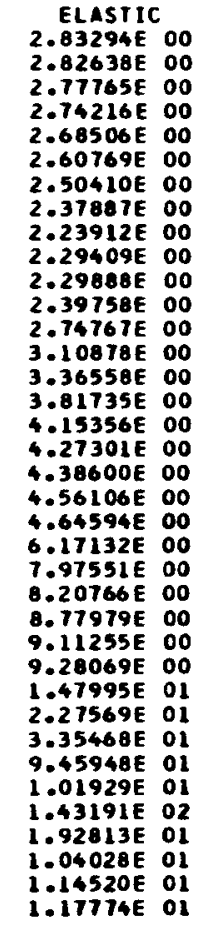 & 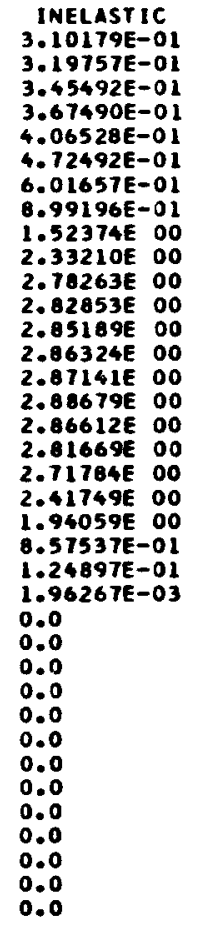 & 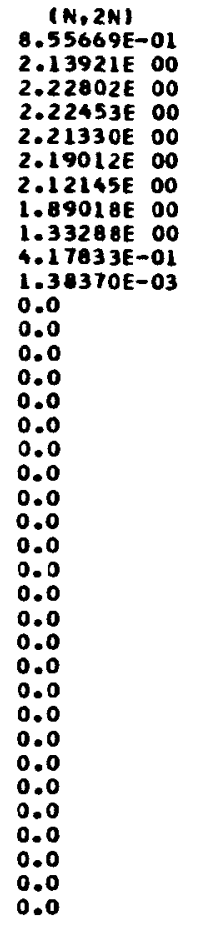 & 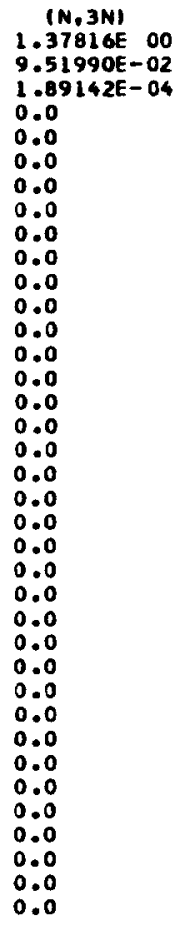 & $\begin{array}{l}\text { ABSORPTION } \\
2.63097 E-02 \\
1.58098 E-02 \\
9.77487 E-03 \\
8.06699 E-03 \\
6.31251 E-03 \\
4.60096 E-03 \\
3.33756 E-03 \\
2.41678 E-03 \\
1.77257 E-03 \\
1.48104 E-03 \\
1.61514 E-03 \\
2.36842 E-03 \\
4.71179 E-03 \\
7.01448 E-03 \\
9.12097 E-03 \\
1.55913 E-02 \\
2.90617 E-02 \\
3.84541 E-02 \\
4.68194 E-02 \\
6.67432 E-02 \\
6.85747 E-02 \\
3.83620 E-02 \\
1.18897 E-01 \\
1.08113 E-01 \\
1.17940 E-01 \\
1.29129 E-01 \\
1.42155 E-01 \\
1.45016 E-01 \\
5.06403 E-02 \\
4.25155 E-02 \\
4.89874 E-02 \\
1.68508 E-01 \\
2.67350 E-01 \\
9.81117 E-01 \\
2.72564 E \text { OD } \\
4.77118 E \\
1.65607 E \\
001\end{array}$ & $\begin{array}{l}\text { (N,GAMAA) } \\
4.21733 E-03 \\
3.82340 E-03 \\
3.66190 E-03 \\
3.60213 E-03 \\
3.50375 E-03 \\
3.31007 E-03 \\
2.96779 E-03 \\
2.39773 E-03 \\
1.77257 E-03 \\
1.48104 E-03 \\
1.61514 E-03 \\
2.36842 E-03 \\
4.71179 E-03 \\
7.01448 E-03 \\
9.12397 E-03 \\
1.55913 E-02 \\
2.90617 E-02 \\
3.84541 E-02 \\
4.68194 E-02 \\
6.67432 E-02 \\
6.85747 E-02 \\
7.83620 E-02 \\
1.18097 E-01 \\
1.08113 E-01 \\
1.17940 E-01 \\
1.29129 E-01 \\
1.42155 E-01 \\
1.45016 E-01 \\
5.06403 E-02 \\
4.25155 E-02 \\
4.89874 E-02 \\
1.68508 E-01 \\
2.67350 E-01 \\
9.81117 E-01 \\
2.72564 E \\
4.77118 E \\
1.65607 E \\
001\end{array}$ & 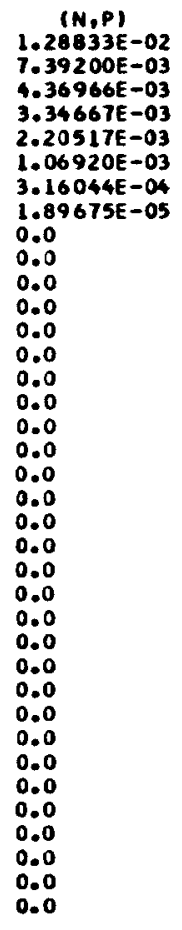 & $\begin{array}{l}\text { (N,ALPHA) } \\
9.20899 E-03 \\
4059436 E-03 \\
1.74332 E-03 \\
1.11819 E-03 \\
6.03597 E-04 \\
2.21699 E-04 \\
5.37265 E-05 \\
7.52708 E-00 \\
0.00 \\
0.00 \\
0.0 \\
0.0 \\
0.00 \\
0.0 \\
0.0 \\
0.0 \\
0.0 \\
0.0 \\
0.00 \\
0.0 \\
0.0 \\
0.0 \\
0.0 \\
0.0 \\
0.00 \\
0.0 \\
0.0 \\
0.00 \\
0.0 \\
0.0 \\
0.0 \\
0.0 \\
0.0 \\
0.0 \\
0.0 \\
0.0 \\
0.0\end{array}$ & 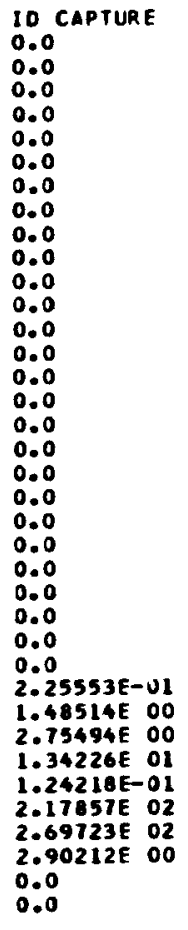 & $\underset{\omega}{\stackrel{5}{+}}$ \\
\hline PHOTON IN & TERACTION CRCS & SECTIONS & & & & & & & & & \\
\hline GROUP & SIG ABS & SIG PE & SIG PP & ENERGY DEP & & & & & & & \\
\hline $\begin{array}{l}1 \\
2 \\
3 \\
4 \\
5 \\
6 \\
7 \\
8 \\
9 \\
10 \\
11 \\
12 \\
13 \\
14 \\
15 \\
16 \\
17 \\
18 \\
19 \\
20 \\
21\end{array}$ & 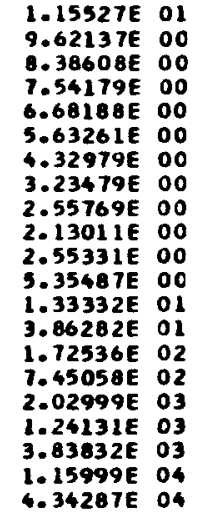 & 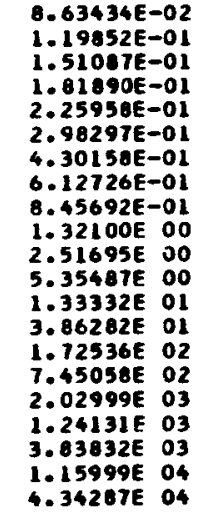 & 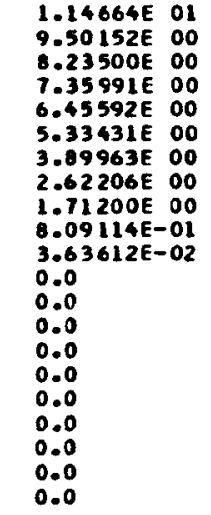 & $\begin{array}{ll}1.55169 E & 02 \\
1.01924 E & 02 \\
7.75907 E & 01 \\
6.32580 E & 01 \\
5.03504 E & 01 \\
3.82815 E & 01 \\
2.73295 E & 01 \\
2.735 \\
2.02673 E & 01 \\
1.64212 E & 01 \\
1.33990 E & 01 \\
1.12764 E & 01 \\
1.04306 E & 01 \\
1.15549 E & 01 \\
1.66853 E & 01 \\
3.63875 E & 01 \\
9.04786 E & 01 \\
1.68316 E & 02 \\
6.93039 E & 01 \\
1.36727 E & 02 \\
2.79559 E & 02 \\
6.08984 E & 02\end{array}$ & & & & & & & \\
\hline
\end{tabular}




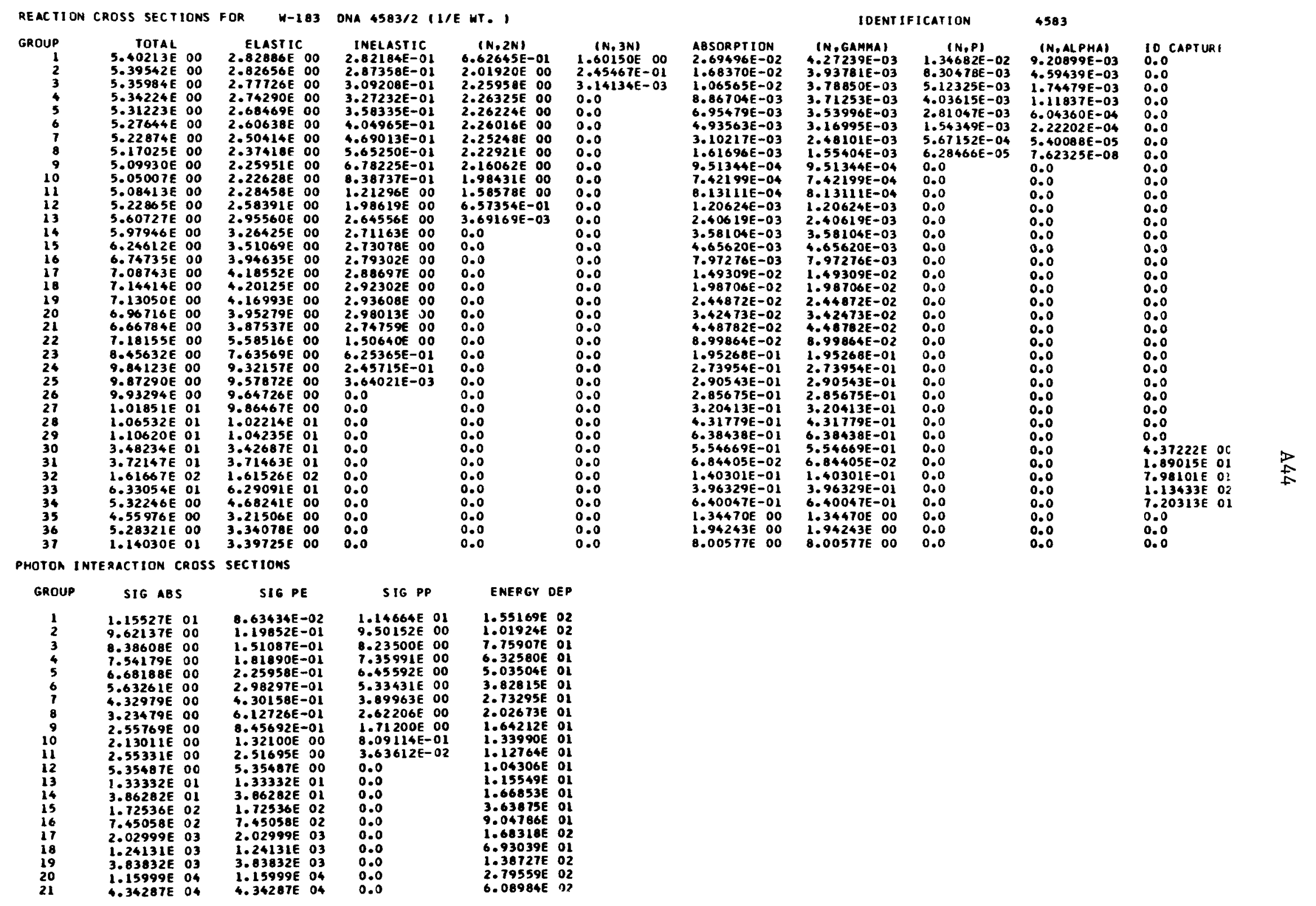




\begin{tabular}{|c|c|c|c|c|c|c|c|c|c|c|}
\hline $\begin{array}{l}\text { REAC IION } \\
\text { GROUP }\end{array}$ & CROSS SECTIONS & FOR $\quad W-184$ & \multicolumn{2}{|c|}{ ONA 4584/2 (1/E NT.) } & & & \multicolumn{2}{|c|}{ IDENTIFICATION } & \multicolumn{2}{|l|}{4584} \\
\hline $\begin{array}{r}\text { GROUP } \\
1 \\
2 \\
3 \\
4 \\
5 \\
6 \\
6 \\
7 \\
8 \\
9 \\
10 \\
11 \\
12 \\
13 \\
14 \\
15 \\
16 \\
17 \\
18 \\
19 \\
20 \\
21 \\
22 \\
23 \\
24 \\
25 \\
26 \\
27 \\
26 \\
29 \\
30 \\
31 \\
32 \\
33 \\
34 \\
35 \\
36 \\
37\end{array}$ & 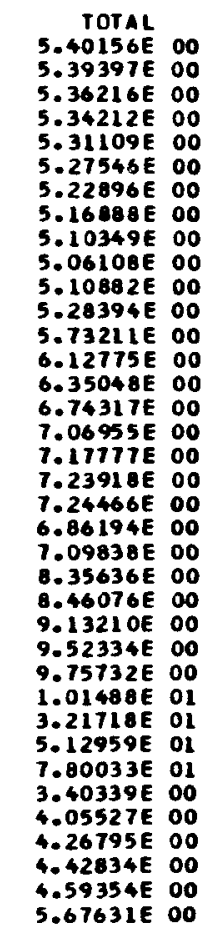 & 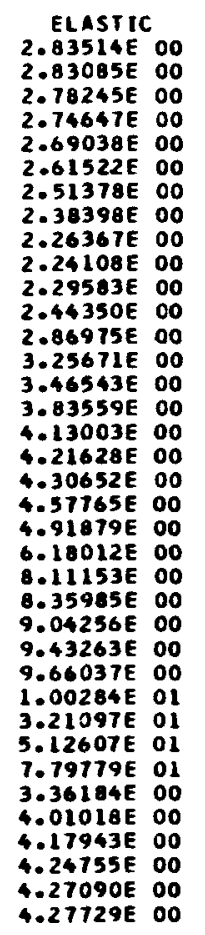 & 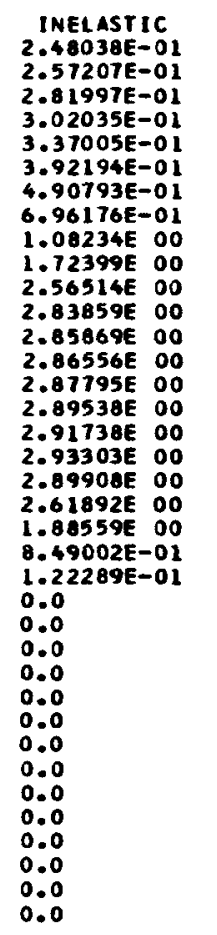 & 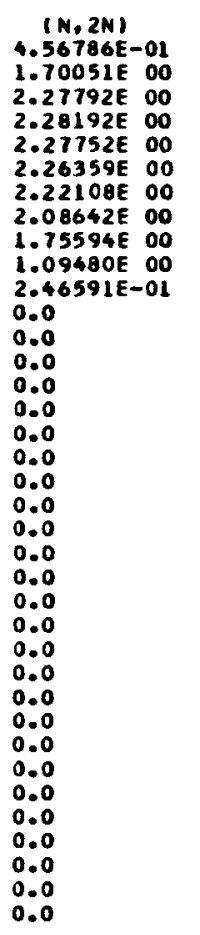 & 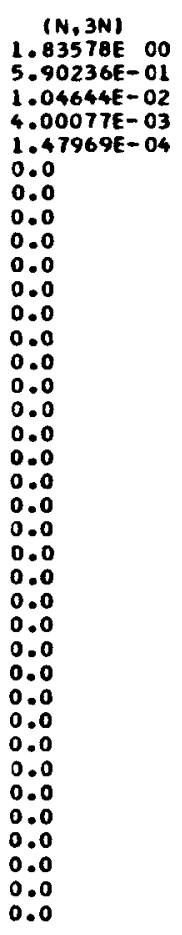 & $\begin{array}{l}\text { ABSORPTION } \\
2.58108 E-02 \\
1.51695 E-02 \\
9.32715 E-03 \\
7.69183 E-03 \\
6.03624 E-03 \\
4.46539 E-03 \\
3.31349 E-03 \\
2.30358 E-03 \\
1.54302 E-03 \\
1.20205 E-03 \\
1.27218 E-03 \\
1.85068 E-03 \\
3.67603 E-03 \\
5.47087 E-03 \\
7.11035 E-03 \\
1.22051 E-02 \\
2.21480 E-02 \\
2.84565 E-02 \\
3.35770 E-02 \\
4.80911 E-02 \\
5.75619 E-02 \\
6.92617 E-02 \\
1.22543 E-01 \\
1.00906 E-01 \\
8.95441 E-02 \\
9.07171 E-02 \\
9.69421 E-02 \\
1.20463 E-01 \\
6.21641 E-02 \\
3.51520 E-02 \\
2.54440 E-02 \\
4.15506 E-02 \\
4.50973 E-02 \\
8.85144 E-02 \\
1.80795 E-01 \\
3.22645 E-01 \\
1.39902 E 00\end{array}$ & $\begin{array}{l}\text { (N,GAMMA) } \\
4.40479 E-03 \\
4.03667 E-03 \\
3.90171 E-03 \\
3.85248 E-03 \\
3.76078 E-03 \\
3.55465 E-03 \\
3.09975 E-03 \\
2.29977 E-03 \\
1.54302 E-03 \\
1.20205 E-03 \\
1.27218 E-03 \\
1.85068 E-03 \\
3.67603 E-03 \\
5.47087 E-03 \\
7.11035 E-03 \\
1.22051 E-02 \\
2.21480 E-02 \\
2.84565 E-02 \\
3.35770 E-02 \\
4.80911 E-02 \\
5.75619 E-02 \\
6.92617 E-02 \\
1.22543 E-01 \\
1.00906 E-01 \\
8.95441 E-02 \\
9.07171 E-02 \\
9.69421 E-02 \\
1.20463 E-01 \\
6.21641 E-02 \\
3.51520 E-02 \\
2.54440 E-02 \\
4.15506 E-02 \\
4.50973 E-02 \\
8.85144 E-02 \\
1.80795 E-01 \\
3.22645 E-01 \\
1.39902 E 00\end{array}$ & $\begin{array}{l}1 \text { (N,P) } \\
1.21971 \mathrm{E}-02 \\
6.53845 E-03 \\
3.68220 E-03 \\
2.7216 E-03 \\
1.67161 E-03 \\
6.89052 E-04 \\
1.60009 E-04 \\
3.72654 E-06 \\
0.00 \\
0.0 \\
0.0 \\
0.00 \\
0.0 \\
0.0 \\
0.00 \\
0.0 \\
0.00 \\
0.0 \\
0.0 \\
0.00 \\
0.0 \\
0.0 \\
0.0 \\
0.0 \\
0.00 \\
0.0 \\
0.0 \\
0.0 \\
0.0 \\
0.00 \\
0.0 \\
0.0 \\
0.00 \\
0.0 \\
0.00 \\
0.0 \\
0.0\end{array}$ & $\begin{array}{l}\text { IN,ALPHAI } \\
9.20899 E-03 \\
4.59433 E-03 \\
1.74324 E-03 \\
1.11819 E-03 \\
6.03853 E-04 \\
2.21699 E-04 \\
5.37265 E-05 \\
7.52708 E-00 \\
0.0 \\
0.0 \\
0.0 \\
0.0 \\
0.0 \\
0.0 \\
0.0 \\
0.0 \\
0.0 \\
0.0 \\
0.0 \\
0.0 \\
0.0 \\
0.00 \\
0.0 \\
0.0 \\
0.0 \\
0.0 \\
0.0 \\
0.0 \\
0.0 \\
0.0 \\
0.00 \\
0.0 \\
0.0 \\
0.00 \\
0.0 \\
0.0 \\
0.0\end{array}$ & $\begin{array}{l}10 \text { rAPTURE } \\
0.0 \\
0.0 \\
0.0 \\
0.0 \\
0.0 \\
0.0 \\
0.0 \\
0.0 \\
0.0 \\
0.0 \\
0.0 \\
0.0 \\
0.0 \\
0.0 \\
0.0 \\
0.0 \\
0.0 \\
0.0 \\
0.0 \\
0.0 \\
0.0 \\
0.0 \\
0.0 \\
0.0 \\
0.0 \\
0.0 \\
0.0 \\
0.0 \\
1.41355 E \\
3.176096 \\
5.92653 E \\
00 \\
1.44300 E-02 \\
0.0 \\
0.0 \\
0.0 \\
0.0 \\
0.0\end{array}$ \\
\hline \multicolumn{11}{|c|}{ PHOTOM INTERACTIOM CRCSS SECTIONS } \\
\hline GROUP & $S I G A B S$ & SIG PE & 516 PP & ENERGY DE & & & & & & \\
\hline $\begin{array}{r}1 \\
2 \\
3 \\
4 \\
5 \\
6 \\
7 \\
8 \\
9 \\
10 \\
11 \\
12 \\
13 \\
14 \\
15 \\
16 \\
17 \\
18 \\
19 \\
20 \\
21\end{array}$ & $\begin{array}{ll}1.15527 E & 01 \\
9.62137 E & 00 \\
8.38608 E & 00 \\
7.58179 E & 00 \\
7.5418188 & 00 \\
6.68188 & 00 \\
5.63261 E & 00 \\
4.32979 E & 00 \\
3.23479 E & 00 \\
2.55769 E & 00 \\
2.13011 E & 00 \\
2.55331 E & 00 \\
5.35487 E & 00 \\
1.33332 E & 01 \\
3.86282 E & 01 \\
1.72536 E & 02 \\
7.45058 E & 02 \\
2.02999 E & 03 \\
1.24131 E & 03 \\
3.83632 E & 03 \\
1.15999 E & 04 \\
4.34287 E & 04\end{array}$ & 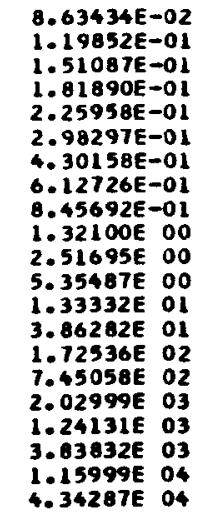 & 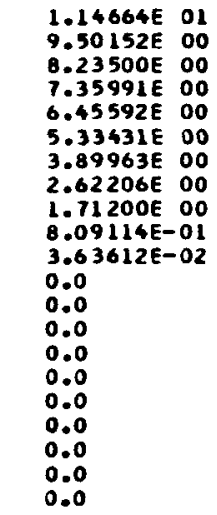 & 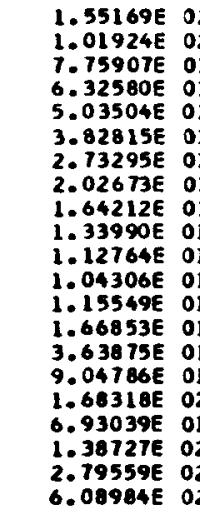 & & & & & & \\
\hline
\end{tabular}


REACTION CROSS SECTIONS FOR W-186 DNA $4586 / 2$ (1/E WT. I

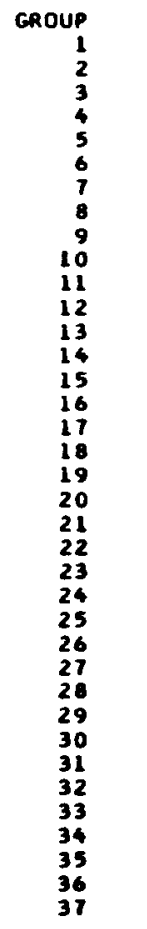

PHOTON INTERACTION CROSS SECTIONS

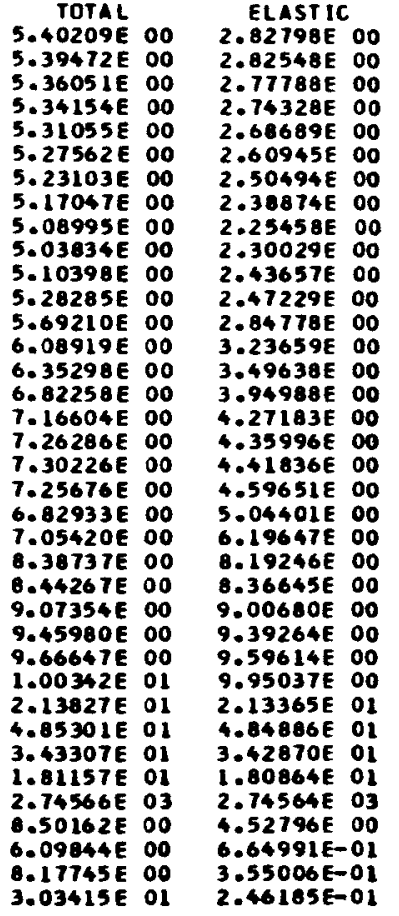

SIG ABS

L.15527E OI $8.38608 E$ OO $7.54179 E 00$ $6.68188 E$ DO $5.63261 E$
$4.32979 E$
400 $4.32979 E$
$3.23479 E$ $3.23479 E$
$2.55769 E$ 2.13011600 $2.55331 E$ OO $5.35487 E$ OO $1.33332 E$ OI $1.72536 E$ O2 $7.45058 E$ O2 1.24131E 03 $3.83832 E$ O $\begin{array}{ll}1.15999 E & 04 \\ 4.34287 E & 04\end{array}$
SIG PE

$8.63434 E-02$ $1.19852 E-01$ 1.81890E-O1 $2.25958 E-01$
$2.98297 E-0$ $4.30158 E-0$ 1. $32100 E 00$ 5.35487E OO 1.33332E O1 $1.72536 E^{02}$ $2.02999 E$ O3 $1.24131 E$ O3 $3.83832 E$ O3 $1.15999 E$ O4
$4.34287 E$ o4 $6.12726 E-01$
$8.45692 E-01$

\begin{tabular}{|c|c|}
\hline 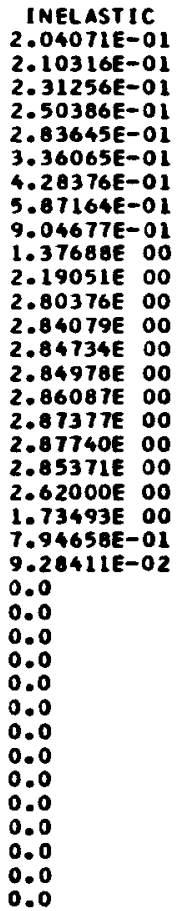 & 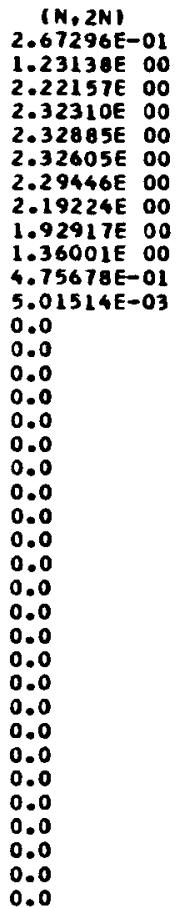 \\
\hline
\end{tabular}

(N, 3N) $.07869 E 00$ $1.21779 \mathrm{E}-01$ $.99266 E-03$ 0.0 0.0 0.0 0.0 0.0 0.0 0.0 0.0 0.0
0.0 0.0 0.0
0.0
0.0 0.0 0.0 0.0
0.0

SIG PP

ENERGY OEP

$1.14664 E$ O1 $1.55169 E$ O2 $9.50152 E$ OD $1.01924 E$ OL 6.32580 E OI $6.45592 E$ OO $5.03504 E$ ol 3.83431E 00 2.62206E 00 2.73295E OL $1.71200 E$ OO $1.64212 E$ OI $8.09114 E-01 \quad 1.33990 E$ OI $3.63612 E-02$ 0.0 0.0 0.0 0.0 0.0

$1.04306 E$ OI
$1.15549 E$ OI

$1.66853 E$ ol

$3.63875 E$ ol

$9.04786 E$ O1
$1.68318 E$ O2

6.93039E ol

$2.79559 \mathrm{E}$
6.02
$6.08984 \mathrm{E}$
IDENTIFICATION

ABSORPTION $2.40472 E-02$ $8.02489 E-03$ $6.55064 E-03$
$5.17922 E-03$ 4.05451E-03 3. $25380 E-03$ $1.52723 E-03$ $1.22624 E-03$ $1.78108 E-03$ $5.53925-03$ $6.82768 E-03$ $1.18315 \mathrm{E}-02$ 2.04411E-02 2.5498E-02 $4.02681 E-02$ $5.03862 E-02$ $6.30735 E-02$ $1.02067 E-01$ $7.62203 E-02$ $6.71598 E-02$ $7.03353 E-02$ $4.62420 \mathrm{E}-02$ $4.14693 E-02$ $4.37441 E-02$ $2.92605 E-02$ $3.97366 E 00$ $5.43345 E 00$ $7.82244 E$
$3.00933 E$ (N, gamaal $4.49801 E-03$
$4.13286 E-03$ $4.00453 E-03$ $3.95826 E-03$ $3.86919 E-03$ $3.18239 E-03$ $1.52723 \mathrm{E}-0$ $1.16580 E-03$ $1.22624 E-03$ L.78108E-0 $5.535925 E-03$ $6.82768 E-03$ $2.04411 E-02$ $2.54989 \mathrm{E}-02$ $3.01849 E-02$ $5.03262 E-02$ $6.30735 E-02$ $1.02067 E-01$ $7.62203 E-02$
$6.67449 E-02$ $6.71598 E-02$ $8.38495 E-02$ $4.62420 E-02$ $4.37441 E-02$ $2.92605 E-02$ 1.97366E 00 $5.43345 E$ OO $7.82244 E$
$3.00953 E$ 4.76271
4586

(N,P)

(N.ALPHA) ..59433E-03 $1.74324 E-03$ $1.11819 E-03$ 7.06420E-04 5.37265E-05 $7.52708 E-0 B$
ID CAPT

0

0.0

0.0 


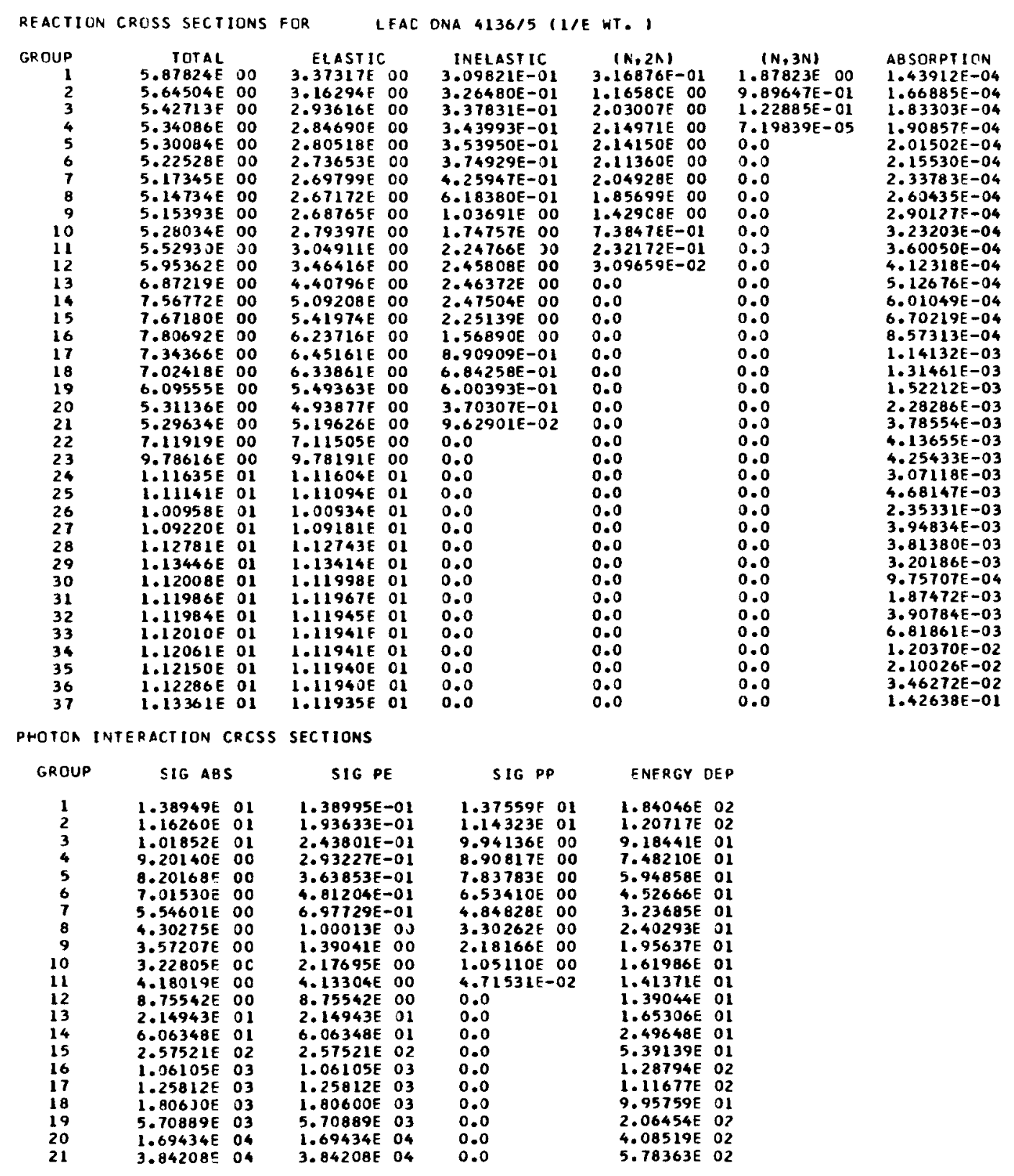

IDENTIFICATION $\quad 4136$ 
ONE OIAENSIUN AVEKAGES FDK U-23B MAT MIBR,MI 37N-21G H3 $1=31.0 K$ INF DIL

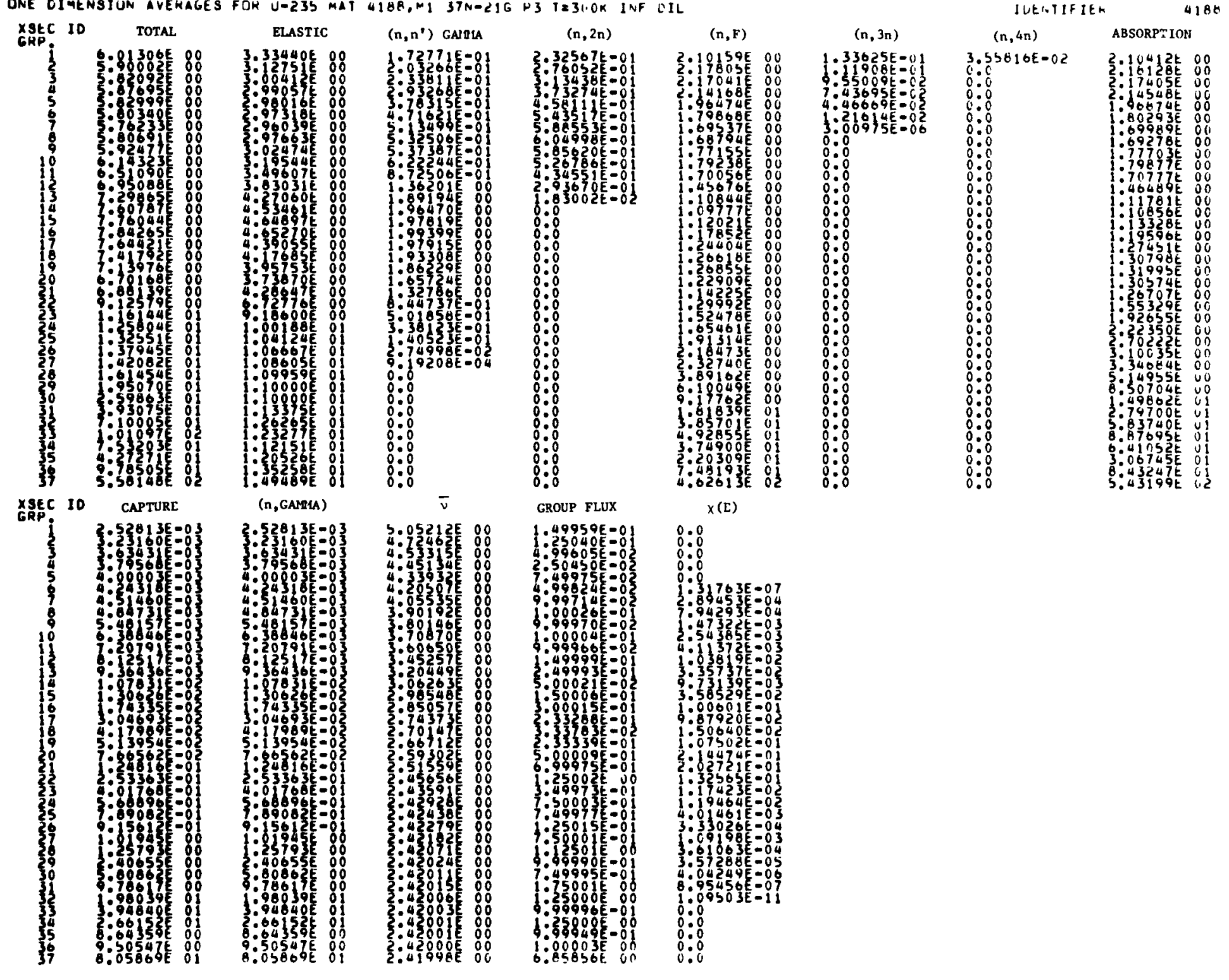

点 
PHOTON INTERACTION CROSS SECTIONS FOR U-235

\begin{tabular}{|c|c|c|c|c|c|c|c|c|}
\hline GRIUP & GFIJUP $L$ & TS (ME.V) & SIG ABS & & SIG PE & SIG PP & ENERGY D & DEP \\
\hline $\begin{array}{r}1 \\
2 \\
3 \\
4 \\
5 \\
6 \\
7 \\
8 \\
9 \\
10 \\
11 \\
12 \\
13 \\
14 \\
15 \\
16 \\
17 \\
18 \\
19 \\
20 \\
21\end{array}$ & 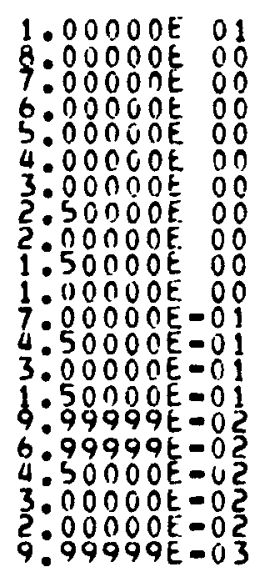 & 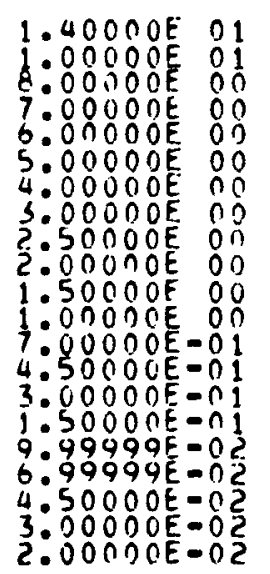 & 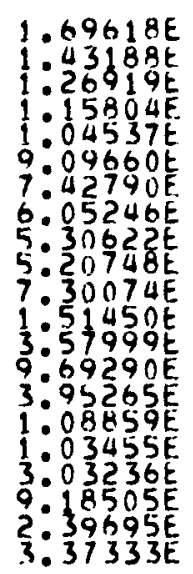 & $\begin{array}{l}01 \\
01 \\
01 \\
01 \\
01 \\
000 \\
00 \\
00 \\
00 \\
00 \\
00 \\
01 \\
01 \\
01 \\
012 \\
03 \\
013 \\
03 \\
03 \\
04 \\
04\end{array}$ & 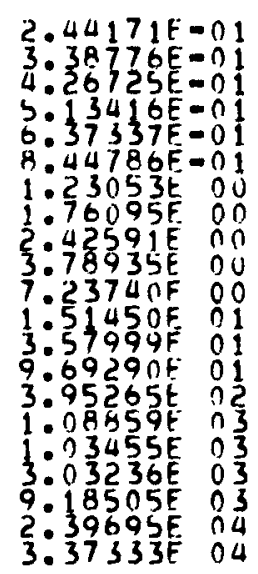 & 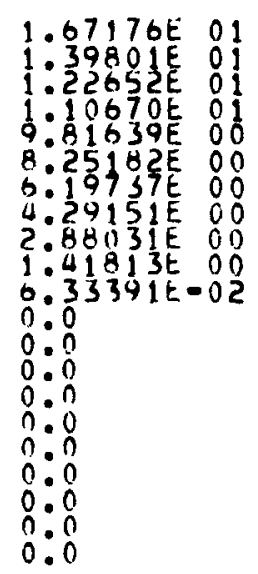 & 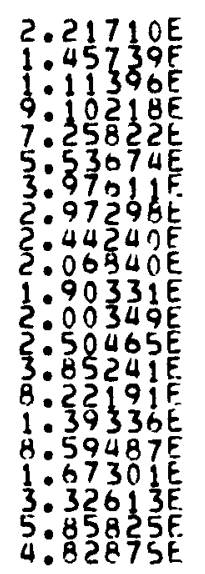 & 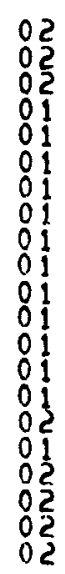 \\
\hline
\end{tabular}


REACTION CROSS SECTIONS FOR L-238 DNA $4187 / 1$ II/E HT.

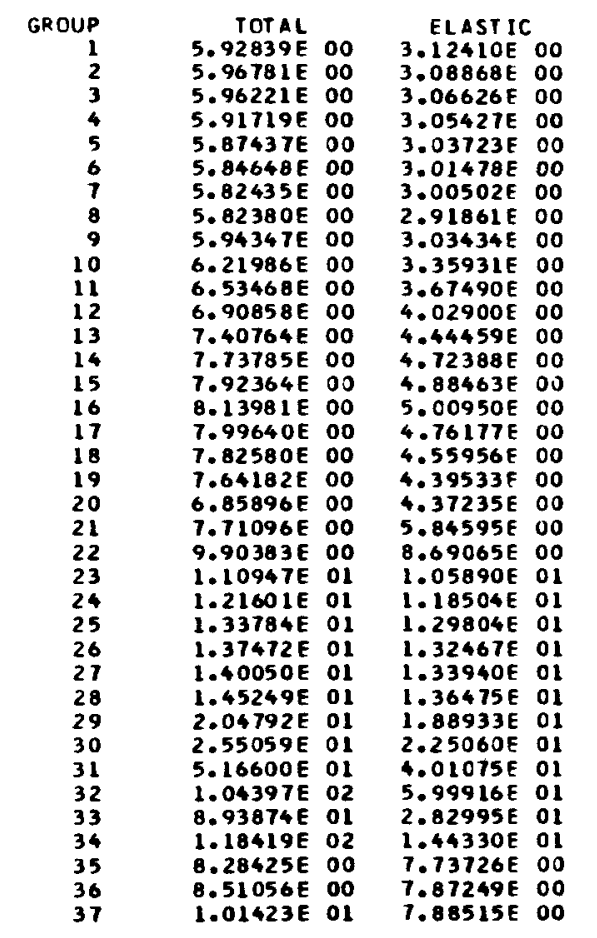

\begin{tabular}{|c|c|}
\hline 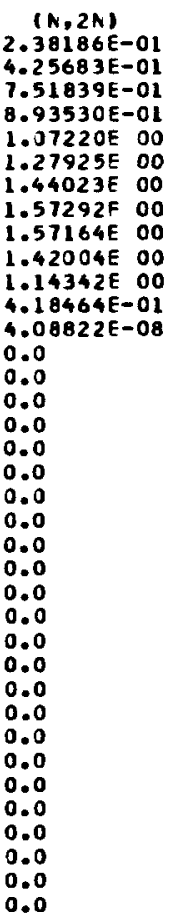 & $\begin{array}{l}1 N, 3 N \mid \\
8.97852 E-C 1 \\
8.30878 E-01 \\
6.10692 E-01 \\
5.08423 E-01 \\
3.78698 E-01 \\
2.23902 E-C 1 \\
4.15166 E-02 \\
0.0 \\
0.0 \\
0.0 \\
0.00 \\
0.0 \\
0.0 \\
0.00 \\
0.0 \\
0.0 \\
0.0 \\
0.0 \\
0.0 \\
0.0 \\
0.0 \\
0.0 \\
0.00 \\
0.0 \\
0.0 \\
0.0 \\
0.0 \\
0.0 \\
0.00 \\
0.0 \\
0.0 \\
0.00 \\
0.0 \\
0.0 \\
0.0 \\
0.0 \\
0.0\end{array}$ \\
\hline
\end{tabular}

EAERGY DEP $2.21710 E$ O2 $1.45739 E$ O2 $1.11396 \mathrm{E} \mathrm{O2}$ $9.10218 \mathrm{E}$ O1 5.53674 E 3.97611E OI $2.97298 E$ OI $2.44240 E$ OI $2.06840 E$ ol 2.00349 OI $2.50465 \mathrm{E}$ OL 3.85241E OI 8. $22191 \mathrm{E}$ O1 $8.59488 E$ OI $1.67301 E 02$ 5.85824E O2
IDENTIFICATIDN

SUM FISSION $.36589 E$
$.31975 E 00$
.3100 $1.22967 E 00$ $1.15476 E$ OO 1.07654E 00 $9.01044 E$ E $9.57337 E-01$ 9.67870E-01 $9.69157 E-01$
$9.54885 E-01$ $.53845 E-01$ $5.22132 E-0$ $5.23782 E-01$
$5.35245 E-01$ $5.35245 E-01$
$5.43701 E-01$ $5.38049 E-0$ (2) $8.04089 E-03$
$6.67602 E-05$ 0.0

0.0

0.0

0.0

0.0
0.0
0.0

0.0

0.0
0.0
0.0

0.0
0.0
0.0
ABSORPTION $1.36825 E 00$ $1.23286 E 00$ $1.08025 E 00$ $1.02450 E 00$ $9.73222 E-01$ $9.74898 E-01$ $9.61259 E-01$ 5.65602E-01 $5.37422 E-01$ $5.45192 E-01$ $5.69652 E-01$ 5. (50740E-01 $1.43526 E-01$ $2.32440 E-01$
$3.97790 E-01$ $5.00520 E-01$ 8.77442E-01 $1.58595 E$ OO $2.99992 E$ OO $1.15525 E$ O1 $6.10879 E$ OI 6.10879E O1 $5.46986 E-01$ $6.38069 E-01$
$2.25716 E 00$

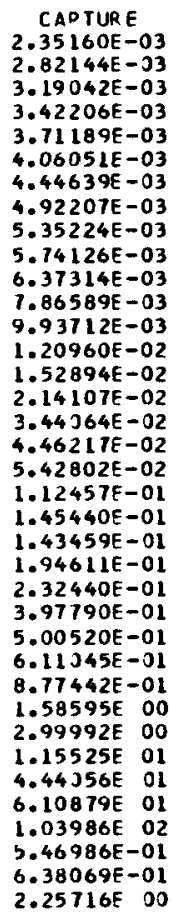

$4.97102 \mathrm{EU} 00 \quad 0.0^{\mathrm{CH}}$ $4.66122 E 00 \quad 0.0$ $4.48373 E$ OO 0.0 4.32576 E 00 0.0 $4.21995 E$ OO $5.85898 E-01$ 4.08496 E 00 5.43594E-O .90014E $00 \quad 1.27725 E-0$ 3.63137E 00 3.60660E-O 3.52414E $00 \quad 5.50937 E-0$ ? 3.40134E OO $1.30794 E-02$ $3.05597 \mathrm{E} 00 \quad 1.08586 \mathrm{E}-02$ 2.98115E 00 3.89613E-0 $2.83711 E$ OO $1.04220 E-01$ 2.70091E 00 9.83450E-02 $2.64872 E 00 \quad 1.47574 E-02$ $2.60954 E$ OO $1.04614 E-J$ $2.45075 E$ EO $1.96166 E-01$ 2.39070E 00 1.32458E-01

0.0 $1.16526 E-02$
$1.15617 E-02$ $3.71224 E-03$ $2.99058 E-04$ $3.12602 E-04$ $3.10679 E-J 5$ 3.87502E-06 $1.07937 E-06$ $3.08495 E-08$ 3..427 $10 \mathrm{E}-10$ $2.64987 E-11$ $3.58666 E-12$
$5.61341 E-13$

$5.20748 E$ OO

$1.51450 E$ OL

$3.57998 E$ OI
$9.69288 E$ OI

$3.95264 E$ O2

$3.03236 \mathrm{E} 03$

$2.39695 \mathrm{E}$ O4

\begin{tabular}{|c|c|}
\hline 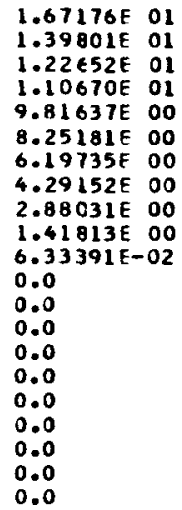 & 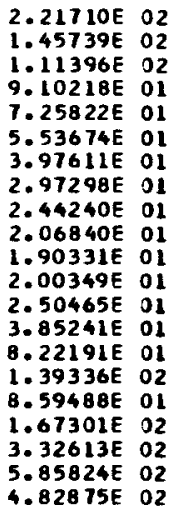 \\
\hline
\end{tabular}


REACTION CRCSS SECTIONS FOR PU-239 DNA 4539/0 (1/E WT.)

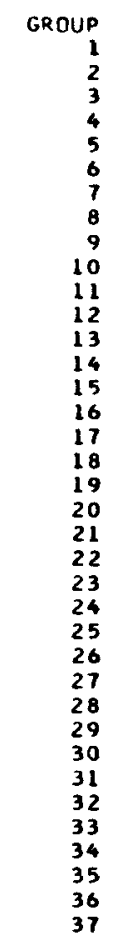

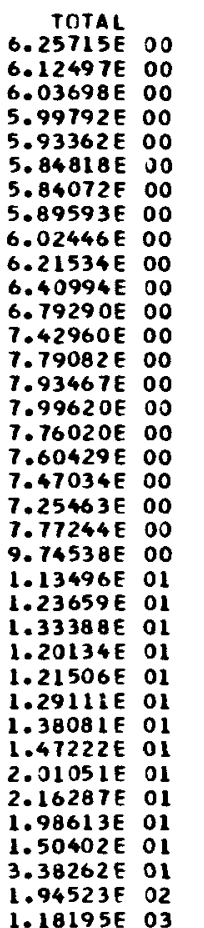

$1.94523 E_{0} 02$ PHOTON INTERACTION CRCSS SECTICNS

\begin{tabular}{ccc} 
GROUP & \multicolumn{2}{c}{ SIG AAS } \\
1 & $1.76103 E$ & 01 \\
2 & $1.48952 E$ & 01 \\
3 & $1.32298 E$ & 01 \\
3 & $1.20927 E$ & 01 \\
4 & $1.09406 E$ & 01 \\
5 & $9.54804 E$ & 00 \\
6 & $9.43233 E$ & 00 \\
7 & $9.815 E$ & 00 \\
8 & 6.4271520 & 00 \\
9 & $5.69272 E$ & 00 \\
10 & $5.65231 E$ & 00 \\
11 & $7.94666 E$ & 00 \\
12 & $1.65986 E$ & 01 \\
13 & $3.94950 E$ & 01 \\
14 & $1.05872 E$ & 02 \\
15 & $4.22847 E$ & 02 \\
16 & $9.72665 E$ & 02 \\
17 & $1.09569 E$ & 03 \\
18 & $3.25378 E$ & 03 \\
19 & $9.95698 E$ & 03 \\
20 & $2.43464 E$ & 04 \\
21 & $3.58525 E$ & 04 \\
& &
\end{tabular}

ELASTIC 3.07541E 0O $3.01883 E 00$ $2.99883 E 00$ $2.96730 \mathrm{E} 00$ $2.94561 E$ OD 3.01740E OO $3.26609 E 00$ $3.46509 E 00$ $3.65655 E 00$ $3.85436 \mathrm{E} 00$ $4.11365 E 00$ $4.39053 E 00$ $4.52852 E 00$ $4.23923 E$ OO 4.07016E 0O 3.99063 E 00 $4.087 C 3 E 00$ $5.09479 E$ OO $9.21278 E$ OO 1.01660E O1 $1.09174 E$ OI 9.93064 EO $1.00581 \mathrm{E}$ OL $1.05918 \mathrm{E}$ ol $1.06662 \mathrm{E}$ O1 1.58126 E 01 $2.02745 E$ O1 $9.32558 E$ OD $1.06105 E$ O $1.23598 E$ ol

INELASTIC $2.18192 E-01$ .22782E-01 $2.24495 E-01$
$2.17793 E-01$
$2.02357 E-O 1$ $2.02359 E-01$ $1.85627 E-01$
$1.75642 E-01$ $.84974 E-01$ . 33943E-01 $3.53769 E-01$
$7.79804 E-01$ .54714E 00 $1.66102 E$ OO $1.65851 E 00$ $1.63156 E 00$ $1.58760 E 00$ $1.52579 E$ DO 9.45469E-01 $5.21094 E-01$ .52926E-01

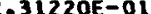
$2.11301 E-01$ $1.61706 E-01$
$.31785 E-02$
0.0 (1.06

0

0.0

$\mathbf{0 . 0}$
0.0

0.0
0.0

0.0

SIG PE

$2.67174 E-01$ $.71935 E-01$ $5.64454 \mathrm{E}-0$ 7.01210E-01 $9.27264 E-01$ $1.34233 E$ OD .65787E 00 .15217E 00 7.87972E DO $1.65986 \mathrm{E}$ O1 $1.54950 E$ OI
$1.05872 E$ O2 4.22847E 02 9.72665E 02 $1.09569 E$ O3 $3.25378 E$ O3 $9.95698 \mathrm{E} \quad 03$ 3.58525E O4

SIG PP

$1.73431 E$ or 1. 152825 OI $1.02394 \mathrm{E} 0$ 8.62C78E OO $4.50806 \mathrm{O}$ $1.50014 E$ DO $6.69367 \mathrm{E}-02$ 0.0
0.0
0.0
0.0 0.0

0.0

0.0
0.0
0.0

0.0
0.0

\begin{tabular}{|c|c|}
\hline 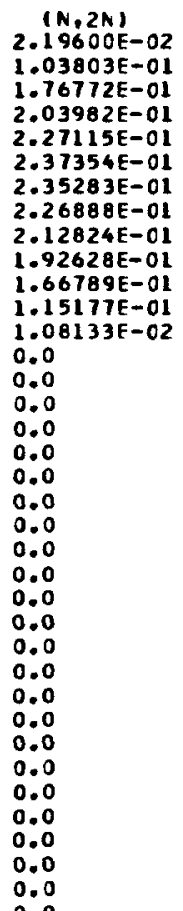 & 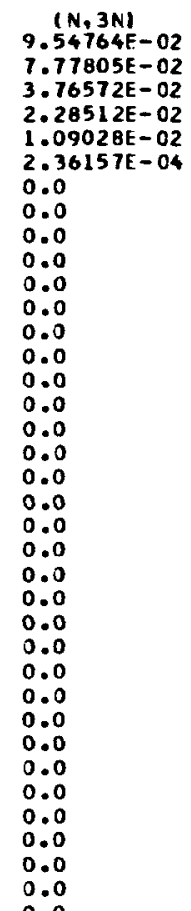 \\
\hline
\end{tabular}
$1.45232 E$ ol $3.03485 \mathrm{E} 00$
$2.29628 E$ O2 $1.51049 \mathrm{E} 02$ $1.15549 E$
$9.44690 E$ 7.53744E 01 $5.75202 E$ Ol
$4.13155 \mathrm{O}$ ol $3.09185 E$ ol 2.54693E OI $2.16583 E$ ol 2.00412E 01 2.13990 EI 2.72099 ol $8.79697 \mathrm{E}$ ol $9.09752 E$ o 1.79419E 02 $3.60460 E$
$5.99567 E$ O2 5.99567E 02
IDENTIFICATION

SUM FISSTON ABSORPTION CAPTURE $2.59895 \mathrm{E} 00 \quad 2.64980 \mathrm{E} 00 \quad 5.08463 E-02$ 2.55686 E 00 2.58094E 0O 2.40799E-32 $2.52943 E$ OO $2.54777 E$ OO $1.83355 E-02$ $2.49728 E$ OO $2.51052 E$ OO $1.32359 E-02$ $2.43366 E$ OO $2.46263 E$ OO $8.96296 E-03$ 2.39550 OO $2.40241 E$ OO $5.91211 E-03$ $2.35836 E$ OO $2.36057 E$ OO $2.32211 E$ DO $2.32368 E$ DO $1.57740 E-03$ $2.23163 E$ OO $2.23284 E$ DO $1.21579 E-03$ $2.04266 E$ E $00 \quad 2.043566$ EO $0.04886 E-04$

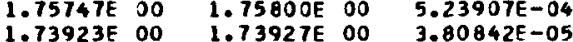
$1.73923 E$ OO $1.80917 E$ DO $1.88931 \mathrm{E} 00$ $1.94526 E 00$ $1.73927 E$ OO 1.80917 00 0.0 $1.88941 E$ 0O $1.06435 E-04$ $1.94653 E$ OO $1.26458 E-03$ $1.95392 E$ OO $5.31465 E-03$ $1.83850 E$ OO $2.01430 E-02$ $1.66467 E 00$ 1.7321 00 OO $1.52874 E$ OO 1.6912 $1.65416 E$ OO $1.93242 E$ 0O $2.78255 E-01$ $1.71985 E$ OO $2.19014 E$ DO $4.70295 E-01$ $1.48759 E$ OO $1.87150 E$ DO $3.83912 E-O$ 1.49647 OO $1.93078 E$ DO $4.34317 E-01$

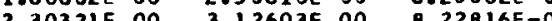
3.01937 E 00 4.05594E DO $1.03657 \mathrm{E} 00$ 3.55507 E 00 4.29248E DO 7.37416E-0 $1.19903 E$ OO $1.35421 E$ OO $1.55182 E-0$ $4.30788 E$ OO 5.49686E OO $1.18898 E$ JJ 4.15697E OO 5.71457E OO $1.55760 \mathrm{E}$ OO

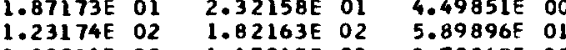




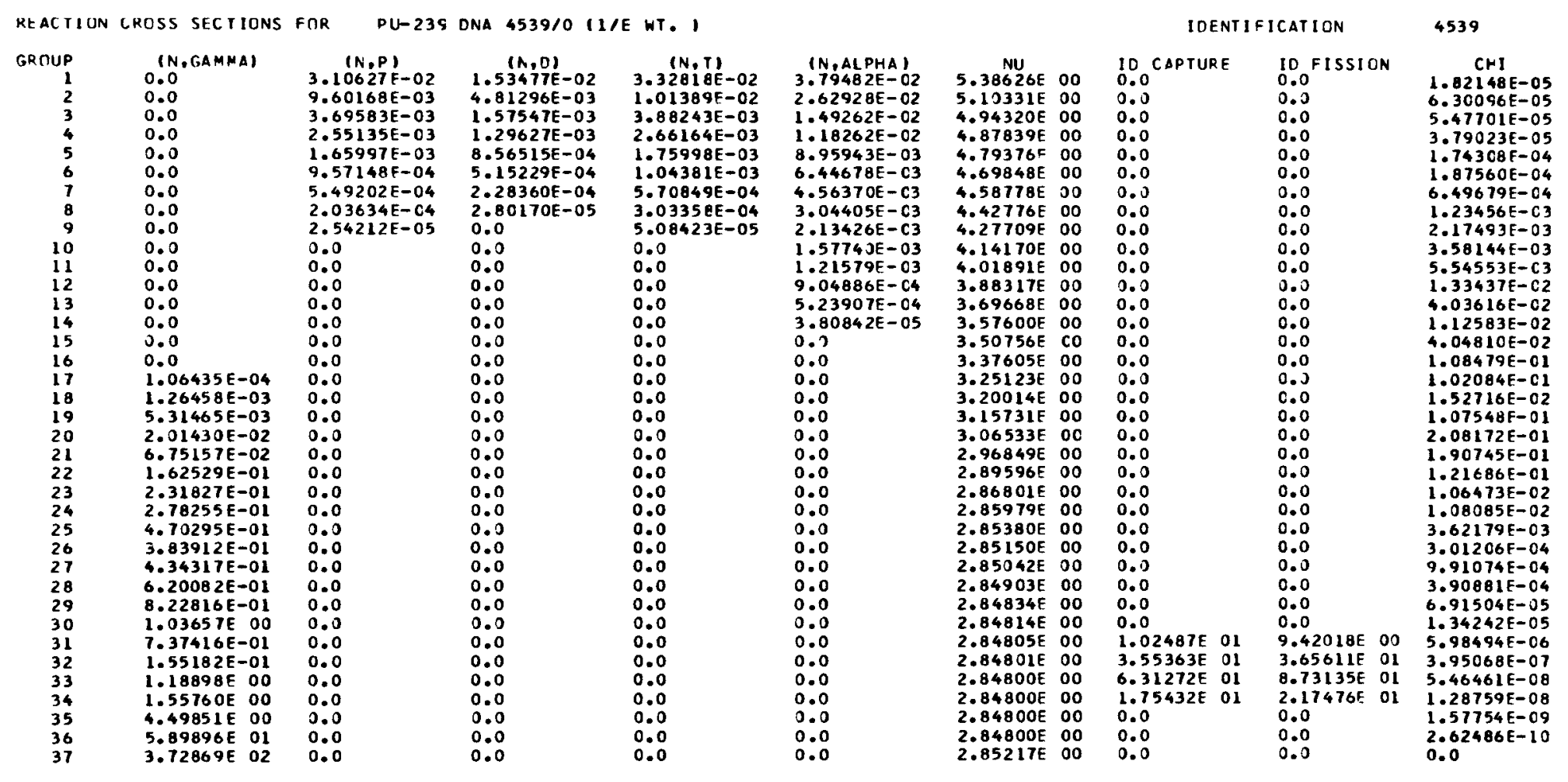


DNE OIMENSION AVERAGES FOR PU-240 MAT 4540,MO 37N-216 P3 T2300K INF DIL

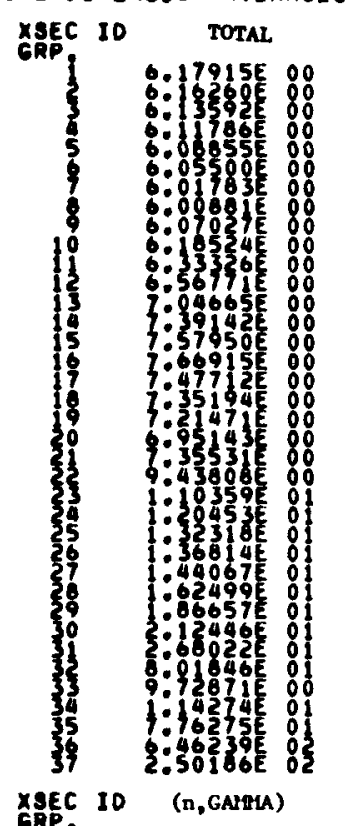

ELASTIC

$(n, 2 n)$
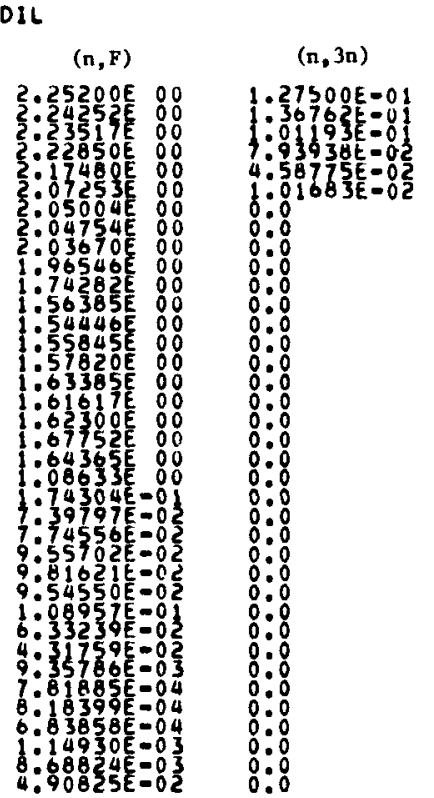

CRP $1.39007 E-094$

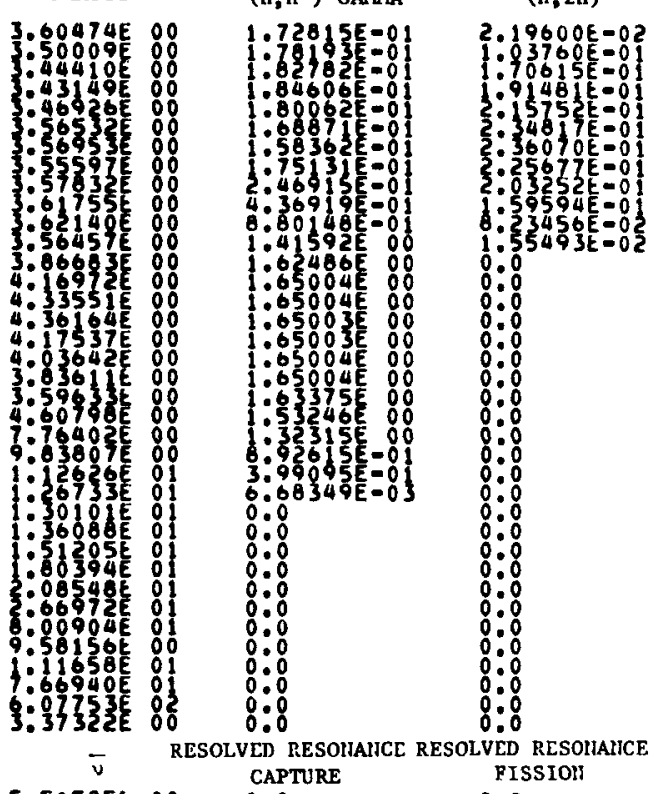

GROUP FLUX

$x(E)$
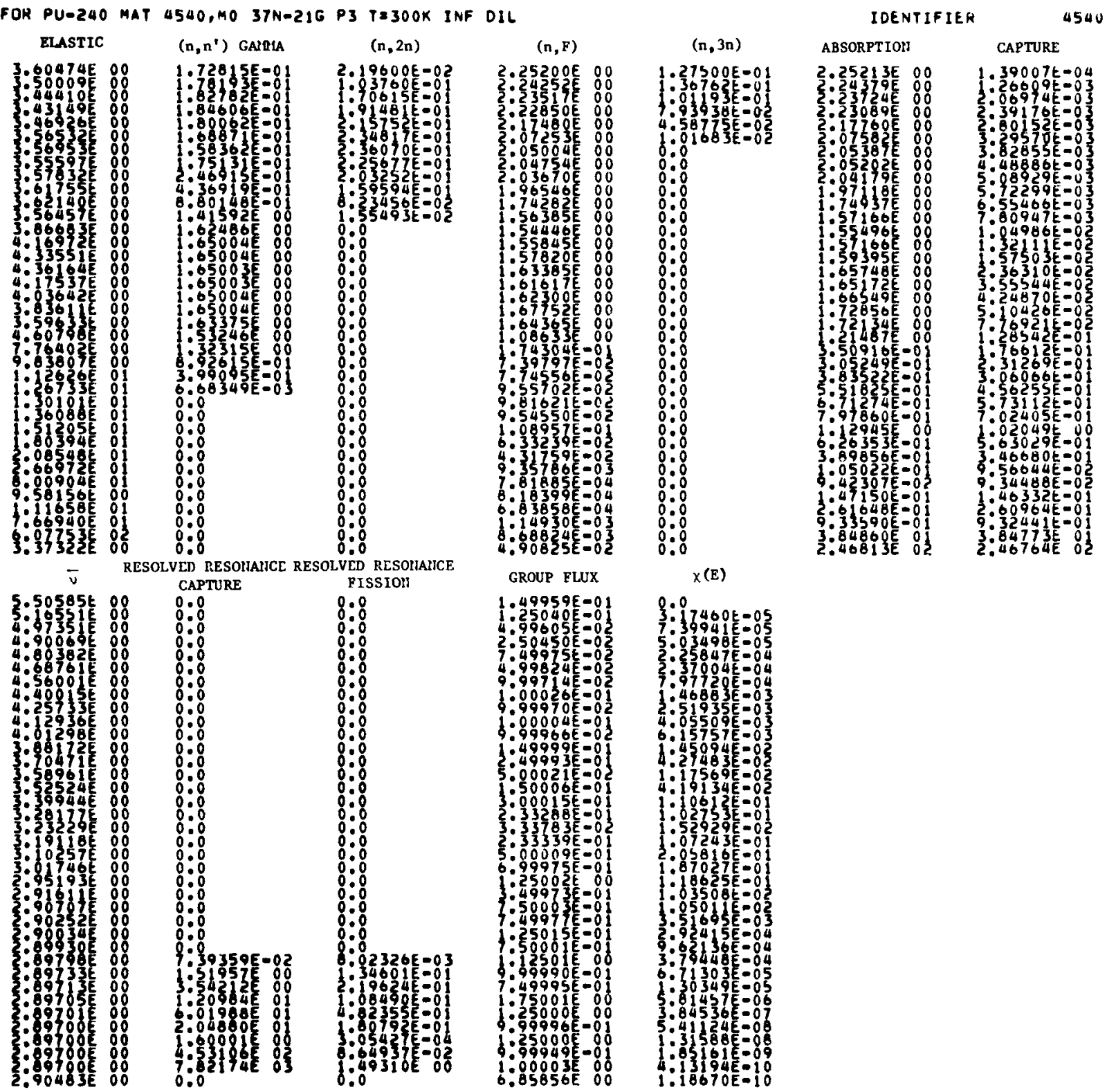
PHOTON INTERACTION CROSS SECTIONS FOR Pu-240

\begin{tabular}{|c|c|c|}
\hline GRUUP & GROUP LI & TS (MEV) \\
\hline $\begin{array}{r}1 \\
2 \\
3 \\
4 \\
5 \\
6 \\
7 \\
8 \\
9 \\
10 \\
11 \\
12 \\
13 \\
14 \\
15 \\
16 \\
17 \\
18 \\
19 \\
30\end{array}$ & 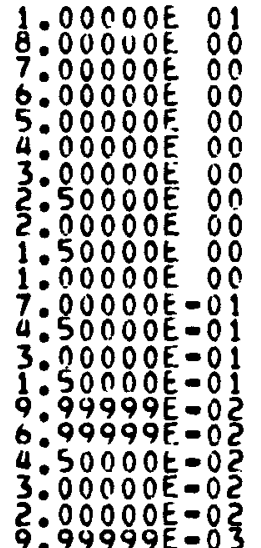 & 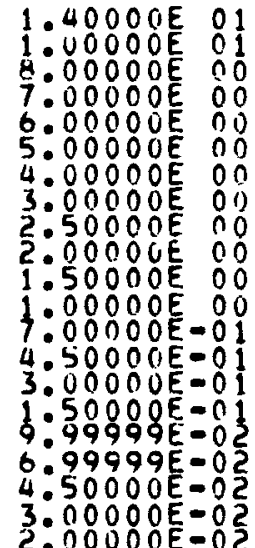 \\
\hline
\end{tabular}

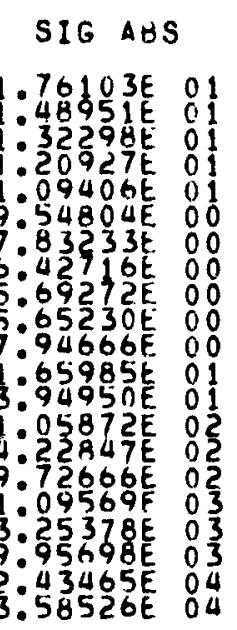

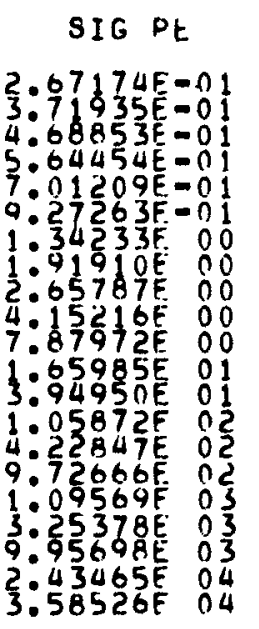

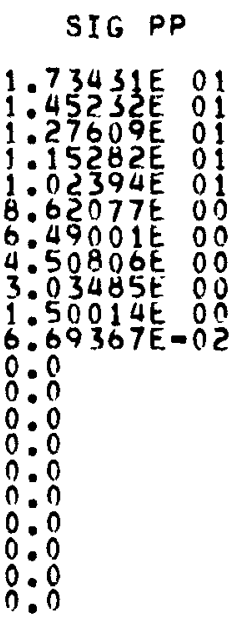

ENERGY DEP 2.29628E 02 $1.15549 E 02$ 9.44690 E 01 $7.53744 F$. 4.13155001 2.54603E $20583 E$ OI ट.00412E 2: 32090 年 01 $4.18603 E$ OI (1) 9.09750 OI $3: 794196$ O2 $5.99568 E$ O2 


\section{APPENDIX B}

Listing of Response Functions

This Appendix contains a listing of response functions included with this library as material 1 . The responses are given as a function of group number (neutron groups 1 through 37; gamma groups 38 through 58) and as a function of position as follows:

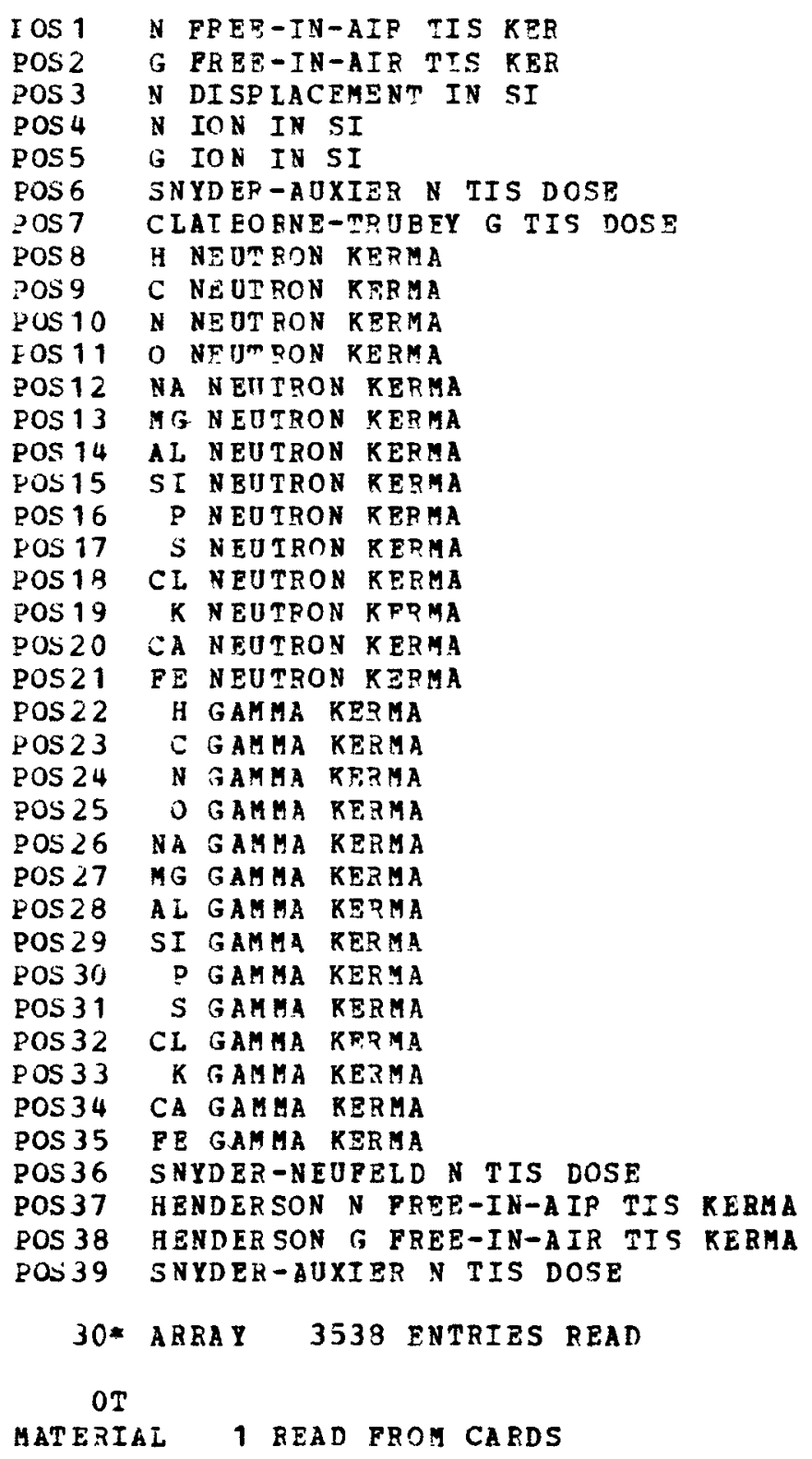

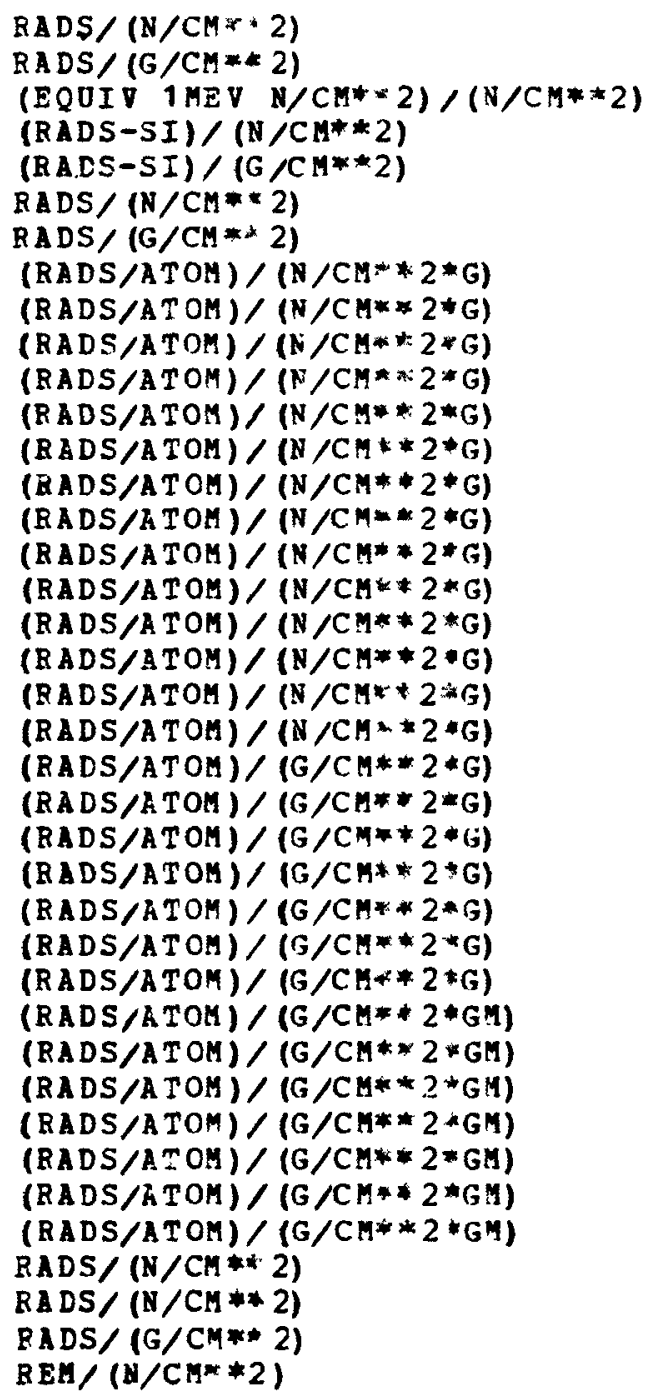




\begin{tabular}{|c|c|c|c|c|}
\hline 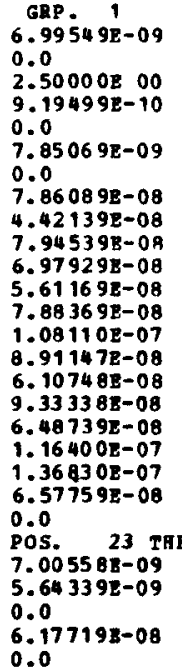 & 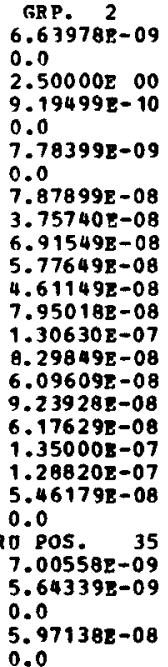 & 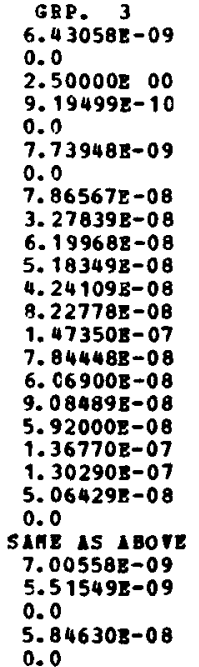 & $\begin{array}{l}\text { GBP } \\
6.32729 \mathrm{E}-09 \\
0.0 \\
2.49800 \mathrm{z} 00 \\
9.19499 \mathrm{z}-10 \\
0.0 \\
7.71169 \mathrm{z}-09 \\
0.0 \\
7.85388 \mathrm{z}-08 \\
3.08860 \mathrm{z}-08 \\
5.86029 \mathrm{z}-08 \\
4.87459 \mathrm{E}-08 \\
4.21659 \mathrm{E}-\mathrm{CB} \\
8.17819 \mathrm{E}-08 \\
1.51770 \mathrm{z}-07 \\
7.57069 \mathrm{E}-08 \\
6.02809 \mathrm{E}-08 \\
9.12929 \mathrm{E}-08 \\
5.79359 \mathrm{E}-08 \\
1.35400 \mathrm{E}-07 \\
1.34120 \mathrm{E}-07 \\
4.88060 \mathrm{~B}-08 \\
0.0\end{array}$ & $\begin{array}{l}\text { GRP } \\
6.18579 \mathrm{E}-09 \\
0.0 \\
2.46300 \mathrm{E} 00 \\
9.19499 \mathrm{E}-10 \\
0.0 \\
7.49489 \mathrm{E}-09 \\
0.0 \\
7.83119 \mathrm{E}-0 \mathrm{~B} \\
3.06530 \mathrm{E}-08 \\
5.47389 \mathrm{E}-08 \\
4.33709 \mathrm{E}-08 \\
4.23339 \mathrm{E}-08 \\
7.81149 \mathrm{E}-08 \\
1.50810 \mathrm{E}-07 \\
7.06348 \mathrm{E}-08 \\
5.80800 \mathrm{E}-08 \\
9.21307 \mathrm{~B}-08 \\
5.59889 \mathrm{E}-08 \\
1.31950 \mathrm{~B}-07 \\
1.41760 \mathrm{E}-07 \\
4.59329 \mathrm{~B}-08 \\
0.0\end{array}$ \\
\hline
\end{tabular}

$\begin{array}{ll}39 & 6.17 \\ 40 & 0.0\end{array}$ $\begin{array}{llcl}0.0 & 0.0 & 0.0 \\ \text { POS. } & 41 \text { THE TI POS. } 61 \text { SAHE AS ABOVE }\end{array}$

Pos.

GRP. 9

20.0

32.22300200

41.159

$\begin{array}{ll}5 & 0.0 \\ 6 & 6.227198-09\end{array}$

70.0

$87.56017 \mathrm{E}-08$

8 4.360195-08

GRP. 10

10 GRP. 11

$0.0 \quad 0.0$

$5.12309 z-09$

GRP. 12

$4.86309 \mathrm{z}-09$

$\begin{array}{cc}2.15700 \mathrm{E} \text { OO } & 2.04600 \mathrm{E} \text { 00 } \\ 1.12000 \mathrm{E}-09 & 1.03300 \mathrm{E}-09\end{array}$

0.0

$6.09929 E-09$

0.0

0.

0.0
$1.82100 \mathrm{~B} 00$

$1.82100 \mathrm{E}$
$7.68599 \mathrm{E}-10$

0.0

5. $974198-09$

0.0

5.81579E- 09

0.0

1. 177805-07

$1.02830 \mathrm{E}-07$

0.0

GRP. 13

$4.559798-09$

1. 70800200

$3.02799 \mathrm{~B}-10$

5.57109B-09

0.0

GRP. 6

GRP. 7

6002498-09

$5.93479 z-09 \quad 5.68159 z-09$

$0.0 \quad 0.0$

$41700 \mathrm{E} 00 \quad 2.36200 \mathrm{E}$ OO $2.29200 \mathrm{E} 00$

$1.011008-09 \quad 1.07900 \mathrm{E}-09 \quad 1.14900 \mathrm{~B}-09$

$0.0 \quad 0.0 \quad 0.0$

$.21409 \mathrm{E}-09 \quad 6.89439 \mathrm{E}-09 \quad 6.48569 \mathrm{E}-09$

$0.0 \quad 0.0$

$7.79659 \mathrm{E}-08 \quad 7.74428 \mathrm{E}-08 \quad 7.66267 \mathrm{E}-08$

$2.94540 \mathrm{E}-08 \quad 2.55440 \mathrm{~B}-08 \quad 2.37600 \mathrm{E}-08$

$\begin{array}{lll}5.00590 \mathrm{E}-08 & 4.25939 \mathrm{E}-08 & 3.66339 \mathrm{z}-08\end{array}$

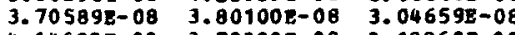

$\begin{array}{lll}4.14689 \mathrm{E}-08 & 3.78380 \mathrm{E}-08 & 3.18960 \mathrm{~B}-08\end{array}$

$\begin{array}{lll}7.28668 \mathrm{E}-08 & 6.28428 \mathrm{E}-08 & 5.14140 \mathrm{E}-08\end{array}$

$1.468108-07 \quad 1.39090 \mathrm{~B}-07 \quad 1.263208-07$

6.46078E-08 $5.76979 \mathrm{z}-08 \quad 4.91169 \mathrm{E}-08$

$5.525798-08 \quad 5.173398-08 \quad 4.69359 z-08$

$9.25708 \mathrm{E}-08$ 9.20618E-08 8.63739E-08

$5.36079-08$ 5.005298-08 4.709008-08

$\begin{array}{lll}5.36079 \mathrm{E}-08 & 5.08529 \mathrm{E}-08 & 4.70900 \mathrm{E}-08 \\ 1.25960 \mathrm{~B}-07 & 1.17400 \mathrm{E}-07 & 1.05430 \mathrm{O}-07\end{array}$

$\begin{array}{lll}1.25960 \mathrm{~B}-07 & 1.17400 \mathrm{E}-07 & 1.05430 \mathrm{E}-07\end{array}$

$\begin{array}{lll}1.47600 \mathrm{E}-07 & 1.48230 \mathrm{E}-07 & 1.44790 \mathrm{E}-07 \\ 4.21249 \mathrm{E}-08 & 3.68339 \mathrm{E}-08 & 3.01630 \mathrm{E}-08\end{array}$ 0.0

0.001080

$7.005588-09$ $5.44049 \mathrm{E}-09$ 0.0

$7.00558 \mathrm{~B}-09$

0.0

$5.15969 \mathrm{~B}-0 \mathrm{~B}$

0.0

7.005588-09 0.0

GRP. 14

$.32029 \mathrm{E}-09$

2.05600200

0.0

5.34319k-09

0.0

$37 \quad .02880 \mathrm{~B}-09 \quad 4.84829 \mathrm{z}-09$

$0.77879=080$

0.00 .0

GRP. 15

255098-09 $4.093798-09$

$0.0 \quad 0.0$

$.67400 \mathrm{E} 00$ 1.33200E 00 0.00 .000
$92.93200 \mathrm{E}-08$

$11 \quad 2.41060 \mathrm{E}-08$

$12 \quad 2.35520 \mathrm{z}-08$

$13 \quad 3.729898-08$

$141.06910 \mathrm{~B}-07$

$15 \quad 4.099698-08$

$\begin{array}{ll}15 & 4.099698-08 \\ 16 & 4.201798-08\end{array}$

$77.662672-08$

$19 \quad 9.402698-08$

$\begin{array}{ll}20 & 1.38850 \mathrm{z}-07 \\ 21 & 2.44900 \mathrm{z}-08\end{array}$

220.0

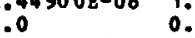

$\begin{array}{rrr}7.43948 z-08 & 7.310398-08 & 7.12878 z-08 \\ 1.677908-08 & 2.342708-08 & 9.521088-09\end{array}$

2.60289B-08

$2.09660 \mathrm{~B}-08$

$1.82430 \mathrm{E}-0$

$2.34270 \mathrm{E}-08$

$1.671405-08$

$2.30590 \mathrm{~B}-08$

$1.61090 \mathrm{E}-\mathrm{CB}$

1. $12850 \mathrm{E}-08$

$1.465508-08$

$1.974908-08$

$1.38150 \mathrm{E}-08$

$9.11037 \mathrm{k}-08$

$3.714108-08$

$3.58479 E-08$
$6.817298-08$

$3.64460 \mathrm{z}-08 \quad 2.19650 \mathrm{z}-08$

2. $95180 \mathrm{~s}-08 \quad 2.36860 \mathrm{E}-08$

6. $05459 \mathrm{E}-0 \mathrm{O}$

2.36860E-08

$4.01569 \mathrm{E}-0 \mathrm{~B}$

$8.37179 z-08$
$1.29230 z-07$

7. $4658 \mathrm{~B}-08$

3. $19329 \mathrm{E}-08$

$3.24180 \mathrm{~L}-08$
$6.46158 \mathrm{E}-\mathrm{CB}$ 0.0

0.0

6.792288-08

1. $19320 \mathrm{~B}-0$

$1.269905-08$

$9.08139 \mathrm{E}-09$

$9.54858 \mathrm{~B}-09$

$4.37139 \mathrm{~B}-0$

$9.978488-0$

$1.69570 \mathrm{~s}-08$
$3.850898-08$

$2.478205-08$

$5.08609 \mathrm{~g}-0 \mathrm{~B}$

$7.76719 \mathrm{~s}-08$

$8.331198-09$

POS. 23 THED POS. 35 SAHE As ABOV

$367.052798-097.10289 \mathrm{E}-09$ 7.036198-09

380.0

7. $03619 z-0$
$.84269 \mathrm{z}-0$

0.0

0.0

6.074298-09

$4.461895-09$

0.0
$4.253398-08$

$\begin{array}{llll}9 & 4.089398-08 & 4.089398-08 & 4.089398-08 \\ 0 & 0.0 & 0.0 & 0.0\end{array}$ POS. 1 THE0 POS. 61 SAHE AS ABOVs

$11160 \mathrm{z}-00$

0.0

POS. GRP. 17

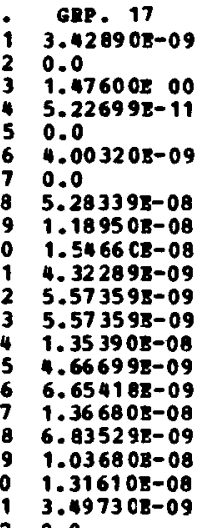

GRP. 18

$3.139598-09$ GRP. 19

0.0

GRP. 19 GRP. 20

0.0

GDP. 20
$2.6457,08-09$

0.0

GRP. 21

2.009008-09

0.0

$\begin{array}{ll}1.37700 \mathrm{E} 00 & 9.61200 \mathrm{E}-01 \\ 4.194998-11 & 2.93699 \mathrm{E}-11\end{array}$

0.0

4.78099

$3.822498-09$

0.0

3.73909s-09

0.0

0.0

$3.52230 \mathrm{z}-09$

0.0

$1.94600 \mathrm{~B}-11$

0.0

$2.94120 z-09$

$\begin{array}{ll}4.730198-08 & 4.03019 \mathrm{E}-08 \\ 8.05969 \mathrm{E}-09 & 6.38509 \mathrm{E}-09\end{array}$

8.98889z-09 7.19039B-09

5.735198-09 6.57119 109

5. $03299 E-09$

$3.94719 E-09$
$9.056298-09$

4. $420608-09$

3.79069 $3-09$

$3.15660 \mathrm{E}-09$
$6.66559 \mathrm{E}-09$

6. $665592-09$
$3.047002-09$

$3.00399 \mathrm{2}-09$

$2.46130 \mathrm{~B}-09$

$2.69760 \mathrm{E}-09$

2. 337008-09

$1.75440 \mathrm{z}-09$

0.0

$5.437892-0$

$7.27208 \mathrm{E}-09 \quad 5.4378$

2.885

0.0

POS. 23 ThRO POS. 35 SAHE AS ABOVE

$\begin{array}{lll}4.478595-09 & 4.342395-09 & 4.228398-09 \\ 3.37210 \mathrm{~B}-09 & 3.14420 \mathrm{z}-09 & 3.08580 \mathrm{z}-09\end{array}$

0.0

$\begin{array}{ll}3.561208-08 & 0.0 \\ 0.0 & 0.0\end{array}$ POS. 1 THRO POS.

0.0

0.0

0

$3.97819 \mathrm{z}-09$

$2.65850 \mathrm{x}-09$

0.0 0.0

$3.58620 \mathrm{~B}-08$

3. 052008-08

4. $801398-09$

3.637608-09

5. 357891-09
$3.664008-09$

2. $22210 \mathrm{~s}-09$

2.392508-09

$2.322108-09$
$1.618108-09$

$1.279108-09$

$1.17320 \mathrm{E}-09$
$1.06950 \mathrm{~B}-09$

$1.174508-09$

8. $455998-10$

0.0

$.514398-08$
$.031408-08$

2. 26210z-08

1.08 1008-08

$7.54909 \mathrm{E}-09$

$3.4798 \mathrm{E}-09$

$8.52048 \mathrm{E}-09$

$8.52048 \mathrm{E}-09$
$1.35170 \mathrm{E}-08$

3. $10660 \mathrm{z}-08$

$1.95660 \mathrm{E}-08$
$4.17779 \mathrm{z}-08$

6.03978 -08

$6.485592-09$

$.69619 \mathrm{E}-09$ $4.26169 z-09$

0.0
$4.29789 \mathrm{E}-08$

3. 34990z-09

2.009105-09

0.0
$3.22760 z-08$

0.0
GRP. 22

$1.24680 \mathrm{E}-09$

0.0

7. 114008-01

1.694108-09

0.0

1.919808-08

2.570208-09

2.00740 -09

$2.88379 B-09$
$1.349608-09$

2.029108-09

$1.265708-09$

1.469908-09

$7.976295-10$
$7.592798-10$

5.204592- 10

5.237898- 10

$5.882892-10$

0.0

1.84200z-09

$1.210108-09$

0.0

0.0 


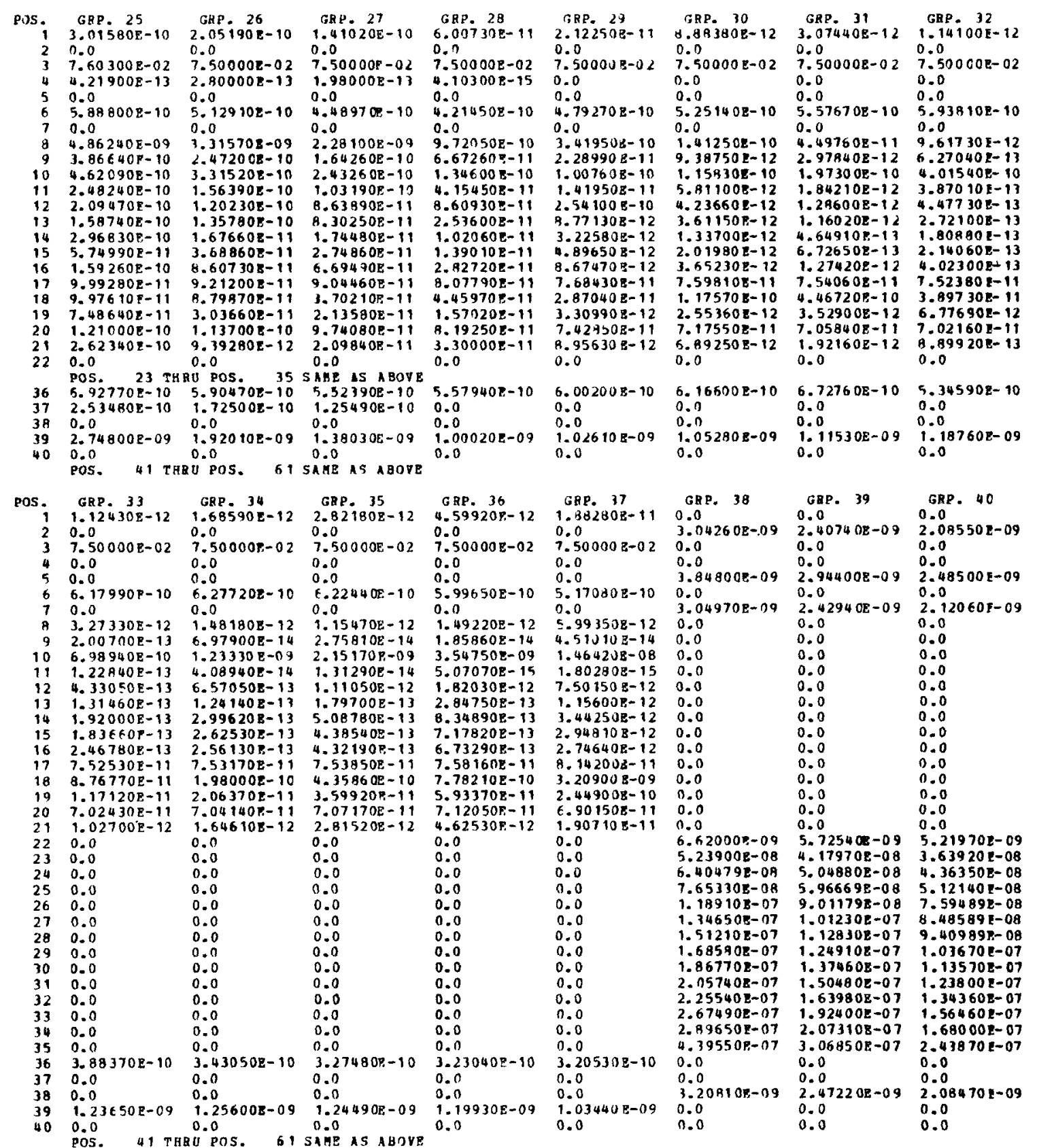




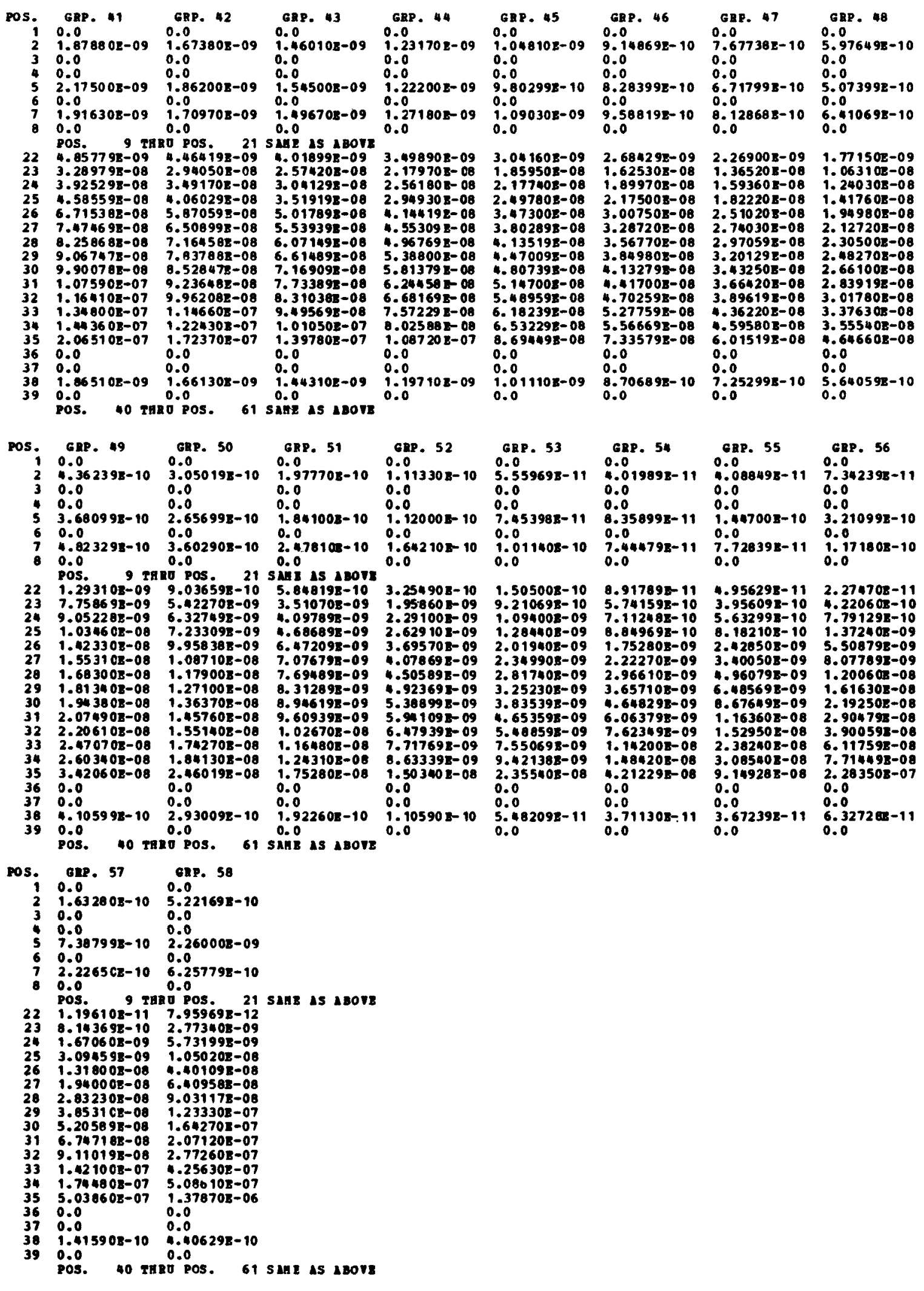




\section{APPENDIX C \\ ARID - A Simple Collapsing Code}

A simple collapsing code, ARID, has been developed from the COMAND module of the AMPX processing code to enable collapse of the $37-21$ library to smaller group structures. The ARID code is included in the library package, and is tailored to receive the 37-21 energy group structure cross-section sets as input data sets. The user then specifies the final group structure and the collapsing spectrum. A (1/E) to thermal Maxwellian neutron spectrum and a flat gamma spectrum are built into the code, or the user can input an arbitrary weighting spectrum, such as that obtained for a specific calculation with the original $37-21$ library. This gives the user the capacity to generate problem-dependent cross-section libraries as desired, or to create smaller problemindependent libraries. The following pages give details on procedures for running ARID and discuss output choices. 
The collapser to go with the DNA Few Group Library is a simplified version of the AMPX COMAND module.

This module, ARID (ANISN Reduction If DNA Few Group Library is Your Base Cross Section Set), assumes the following:

1. An ANISN Iibrary in card image or binary format is mounted on logical unit 20. It has the ANISN $\mathrm{P}_{\ell}$ decks stacked with control cards interspersed as if one were getting ready to run ALC1 to make an ANISN 1ibrary.

2. A collapsed library will be written in ANISN binary format on logical unit 18. These can be punched onto cards in the ANISN free-form BCD format on logical 7.

3. ARID assumes:

a. total number of groups on DNA library is 58 (37 neutron and 21 gamma),

b. the total cross section is in position 3, c. the within group cross section is in position 4 , d. the table length of the DNA 1ibrary is 61 .

4. One can selectively collapse the data over a built-in spectrum, or the user may override the built-in data by inputting his own. The built-in spectrum consists of a $1 / E$ variation joined to a thermal Maxwellian (300k) for neutrons and has a flat variation for gamma groups.

The data required are as follows:

$1 \$ \$$ Collapsing Case Description 
1. IGMF - total number of groups in broad structure.

2. IHTF - position of $\sigma_{\mathrm{T}}$ in broad structure.

3. IHSF - position of $\sigma_{g g}$ in broad structure.

4. IHMF - table length in broad structure.

5. IEDIT - 0, almost no edit,

1 , edit collapsed data,

2, edit fine group and collapsed data.

6. NSPEC - spectrum number to use in collapsing (input a zero).

7. $\mathrm{NPNCH}-0$, no punch,

1, punch collapsed cross sections in ANISN free-form format.

8. NFM - -1, DNA Few Group Library is in ANISN binary format,

0 , DNA Few Group Library is in ANISN BCD free-form format,

1, DNA Few Group Library is in ANISN BCD fixed form format.

T Terminate Block 1

$3 * *$ Weighting Spectrum (This will override a selected spectrum.)

$4 \$$ Broad Group Numbers by Fine Group

This corresponds to the $28 \$$ array in ANISN or $51 \$$ array in XSDRN.

A zero entry will suppress a group.

T Terminate Case Data

Note that one can use ARID to convert ANISN library formats, either BCD to binary or binary to $\mathrm{BCD}$. 
APPENDIX D

Energy Group Structure for $129 n-43 \gamma$ Cross-Section Set

\begin{tabular}{c} 
Group \\
\hline 1 \\
2 \\
3 \\
4 \\
5 \\
6 \\
7 \\
8 \\
9 \\
10 \\
11 \\
12 \\
13 \\
14 \\
15 \\
16 \\
17 \\
18 \\
19 \\
20 \\
21 \\
22 \\
23 \\
24 \\
25 \\
26 \\
27 \\
28 \\
29 \\
30 \\
31 \\
32 \\
33 \\
34 \\
35 \\
36 \\
37 \\
38 \\
39 \\
40 \\
41 \\
42 \\
43 \\
44 \\
45 \\
46
\end{tabular}

Energy Range
$1.4000 \mathrm{E} 07-1.4500 \mathrm{E} 07$
$1.3250 \mathrm{E} 07-1.4000 \mathrm{E} 07$
$1.2500 \mathrm{E} 07-1.3250 \mathrm{E} 07$
$1.1750 \mathrm{E} 07-1.2500 \mathrm{E} 07$
$1.1500 \mathrm{E} 07-1.1750 \mathrm{E} 07$
$1.1250 \mathrm{E} 07-1.1500 \mathrm{E} 07$
$1.1000 \mathrm{E} 07-1.1250 \mathrm{E} 07$
$1.0800 \mathrm{E} 07-1.100 \mathrm{E} 07$
$1.0500 \mathrm{E} 07-1.0800 \mathrm{E} 07$
$1.0000 \mathrm{E} 07-1.0500 \mathrm{E} 07$
$9.7500 \mathrm{E} 06-1.000 \mathrm{E} 07$
$9.5000 \mathrm{E} 06-9.7500 \mathrm{E} 06$
$8.9000 \mathrm{E} 06-9.500 \mathrm{E} 06$
$8.6000 \mathrm{E} 06-8.9000 \mathrm{E} 06$
$8.4000 \mathrm{E} 06-8.6000 \mathrm{E} 06$
$8.2000 \mathrm{E} 06-8.4000 \mathrm{E} 06$
$8.1000 \mathrm{E} 06-8.2000 \mathrm{E} 06$
$8.0000 \mathrm{E} 06-8.1000 \mathrm{E} 06$
$7.9000 \mathrm{E} 06-8.0000 \mathrm{E} 06$
$7.7500 \mathrm{E} 06-7.9000 \mathrm{E} 06$
$7.5000 \mathrm{E} 06-7.7500 \mathrm{E} 06$
$7.2500 \mathrm{E} 06-7.5000 \mathrm{E} 06$
$7.1000 \mathrm{E} 06-7.2500 \mathrm{E} 06$
$7.0000 \mathrm{E} 06-7.1000 \mathrm{E} 06$
$6.8500 \mathrm{E} 06-7.0000 \mathrm{E} 06$
$6.7000 \mathrm{E} 06-6.8500 \mathrm{E} 06$
$6.5700 \mathrm{E} 06-6.7000 \mathrm{E} 06$
$6.4300 \mathrm{E} 06-6.5700 \mathrm{E} 06$
$6.2500 \mathrm{E} 06-6.4300 \mathrm{E} 06$
$5.7500 \mathrm{E} 06-6.2500 \mathrm{E} 06$
$5.5200 \mathrm{E} 06-5.7500 \mathrm{E} 06$
$5.2000 \mathrm{E} 06-5.5200 \mathrm{E} 06$
$5.0700 \mathrm{E} 06-5.2000 \mathrm{E} 06$
$5.0000 \mathrm{E} 06-5.0700 \mathrm{E} 06$
$4.9200 \mathrm{E} 06-5.0000 \mathrm{E} 06$
$4.8000 \mathrm{E} 06-4.9200 \mathrm{E} 06$
$4.7200 \mathrm{E} 06-4.8000 \mathrm{E} 06$
$4.6500 \mathrm{E} 06-4.7200 \mathrm{E} 06$
$4.4500 \mathrm{E} 06-4.6500 \mathrm{E} 06$
$4.3000 \mathrm{E} 06-4.4500 \mathrm{E} 06$
$4.1400 \mathrm{E} 06-4.3000 \mathrm{E} 06$
$4.0500 \mathrm{E} 06-4.1400 \mathrm{E} 06$
$3.9500 \mathrm{E} 06-4.0500 \mathrm{E} 06$
$3.8000 \mathrm{E} 06-3.9500 \mathrm{E} 06$
$3.6000 \mathrm{E} 06-3.8000 \mathrm{E} 06$
$3.4500 \mathrm{E} 06-3.6000 \mathrm{E} 06$

Lethargy Range

$-0.372--0.336$

$-0.336--0.281$

$-0.281--0.223$

$-0.223--0.161$

$-0.161--0.140$

$-0.140--0.118$

$-0.118--0.095$

$-0.095--0.077$

$-0.077--0.049$

$-0.049-0.0$

$0.0-0.025$

$0.025-0.051$

$0.051-0.117$

$0.117-0.151$

$0.151-0.174$

$0.174-0.198$

$0.198-0.211$

$0.211-0.223$

$0.223-0.236$

$0.236-0.255$

$0.255-0.288$

$0.288-0.322$

$0.322-0.342$

$0.342-0.357$

$0.357-0.378$

$0.378-0.400$

$0.400-0.420$

$0.420-0.442$

$0.442-0.470$

$0.470-0.553$

$0.553-0.594$

$0.594-0.654$

$0.654-0.679$

$0.679-0.693$

$0.693-0.709$

$0.709-0.734$

$0.734-0.751$

$0.751-0.766$

$0.766-0.810$

$0.810-0.844$

$0.844-0.882$

$0.882-0.904$

$0.904-0.929$

$0.929-0.968$

$0.968-1.022$

$1.022-1.064$ 
Energy Group Structure (Cont'd.)

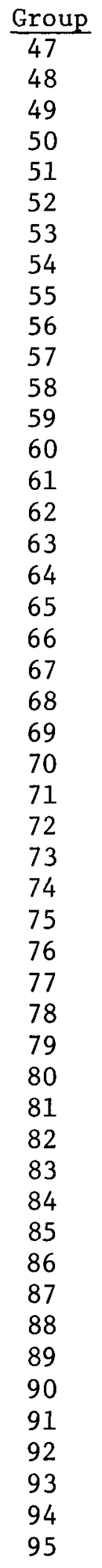

Energy Range
3.300 EE06 - $3.4500 E 06$
$3.1500 E 06-3.3000 E 06$
$3.0000 E 06-3.1500 E 06$
$2.9400 E 06-3.0000 E 06$
$2.8700 E 06-2.9400 E 06$
$2.7800 E 06-2.8700 E 06$
$2.7200 E 06-2.7800 E 06$
$2.4500 E 06-2.7200 E 06$
$2.4000 E 06-2.4500 E 06$
$2.3800 E 06-2.4000 E 06$
$2.3600 E 06-2.3800 E 06$
$2.3400 E 06-2.3600 E 06$
$2.3200 E 06-2.3400 E 06$
$2.3000 E 06-2.3200 E 06$
$2.2600 E 06-2.3000 E 06$
$2.1800 E 06-2.2600 E 06$
$1.9700 E 06-2.1800 E 06$
$1.8500 E 06-1.9700 E 06$
$1.8200 E 06-1.8500 E 06$
$1.7500 E 06-1.8200 E 06$
$1.6600 E 06-1.7500 E 06$
$1.6300 E 06-1.6600 E 06$
$1.5700 E 06-1.6300 E 06$
$1.4500 E 06-1.5700 E 06$
$1.3900 E 06-1.4500 E 06$
$1.3300 E 06-1.3900 E 06$
$1.2900 E 06-1.3300 E 06$
$1.2700 E 06-1.2900 E 06$
$1.2450 E 06-1.2700 E 06$
$1.2200 E 06-1.2450 E 06$
$1.2000 E 06-1.2200 E 06$
$1.1500 E 06-1.2000 E 06$
$1.1000 E 06-1.1500 E 06$
$1.0500 E 06-1.1000 E 06$
$9.8000 E 05-1.0500 E 06$
$9.3000 E 05-9.8000 E 05$
$8.3000 E 05-9.3000 E 05$
$7.3000 E 05-8.3000 E 05$
$6.3000 E 05-7.3000 E 05$
$5.8000 E 05-6.3000 E 05$
$5.0000 E 05-5.8000 E 05$
$4.0000 E 05-5.0000 E 05$
$2.5000 E 05-4.0000 E 05$
$1.5500 E 05-2.5000 E 05$
$1.3000 E 05-1.5500 E 05$
$1.1100 E 05-1.3000 E 05$
$8.2500 E 04-1.1100 E 05$
$8.1000 E 04-8.2500 E 04$
$7.2000 E 04-8.1000 E 04$

Lethargy Range

$1.064-1.109$

$1.109-1.155$

$1.155-1.204$

$1.204-1.224$

$1.224-1.248$

$1.248-1.280$

$1.280-1.302$

$1.302-1.406$

$1.406-1.427$

$1.427-1.435$

$1.435-1.444$

$1.444-1.452$

$1.452-1.461$

$1.461-1.470$

$1.470-1.487$

$1.487-1.523$

$1.523-1.625$

$1.625-1.687$

$1.687-1.704$

$1.704-1.743$

$1.743-1.796$

$1.796-1.814$

$1.814-1.852$

$1.852-1.931$

$1.931-1.973$

$1.973-2.017$

$2.017-2.048$

$2.048-2.064$

$2.064-2.083$

$2.083-2.104$

$2.104-2.120$

$2.120-2.163$

$2.163-2.207$

$2.207-2.254$

$2.254-2.323$

$2.323-2.375$

$2.375-2.489$

$2.489-2.617$

$2.617-2.765$

$2.765-2.847$

$2.847-2.996$

$2.996-3.219$

$3.219-3.689$

$3.689-4.167$

$4.167-4.343$

$4.343-4.501$

$4.501-4.798$

$4.798-4.816$

$4.816-4.934$ 
Energy Group Structure (Cont'd.)

\begin{tabular}{|c|c|}
\hline Group & Energy Range \\
\hline 96 & $6.800 \overline{0 \mathrm{E} 04-7.2000 \mathrm{E} 04}$ \\
\hline 97 & $4.0860 \mathrm{E} 04-6.8000 \mathrm{E} 04$ \\
\hline 98 & $3.5000 \mathrm{E} 04-4.0860 \mathrm{E} 04$ \\
\hline 99 & $3.0000 \mathrm{E} 04-3.5000 \mathrm{E} 04$ \\
\hline 100 & $2.7000 \mathrm{E} 04-3.0000 \mathrm{E} 04$ \\
\hline 101 & $2.5000 \mathrm{E} 04-2.7000 \mathrm{E} 04$ \\
\hline 102 & $2.3000 \mathrm{E} 04-2.5000 \mathrm{E} 04$ \\
\hline 103 & $1.9300 \mathrm{E} 04-2.3000 \mathrm{E} 04$ \\
\hline 104 & $1.5030 \mathrm{E} 04-1.9300 \mathrm{E} 04$ \\
\hline 105 & $9.1100 \mathrm{E} 03-1.5030 \mathrm{E} 04$ \\
\hline 106 & $5.5300 \mathrm{E} 03-9.1100 \mathrm{E} 03$ \\
\hline 107 & $3.3500 \mathrm{E} 03-5.5300 \mathrm{E} 03$ \\
\hline 108 & $2.0300 \mathrm{E} 03-3.3500 \mathrm{E} 03$ \\
\hline 109 & $1.2300 \mathrm{E} 03-2.0300 \mathrm{E} 03$ \\
\hline 110 & $9.6100 \mathrm{E} 02-1.2300 \mathrm{E} 03$ \\
\hline 111 & $7.4900 \mathrm{E} 02-9.6100 \mathrm{E} 02$ \\
\hline 112 & $5.8300 \mathrm{E} 02-7.4900 \mathrm{E} 02$ \\
\hline 113 & $3.5300 \mathrm{E} 02-5.8300 \mathrm{E} 02$ \\
\hline 114 & $2.7500 \mathrm{E} 02-3.5300 \mathrm{E} 02$ \\
\hline 115 & $1.6700 \mathrm{E} 02-2.7500 \mathrm{E} 02$ \\
\hline 116 & $1.0100 \mathrm{E} 02-1.6700 \mathrm{E} 02$ \\
\hline 117 & $7.8900 \mathrm{E} 01-1.0100 \mathrm{E} 02$ \\
\hline 118 & $4.7800 \mathrm{E} 01-7.8900 \mathrm{E} 01$ \\
\hline 119 & $2.9000 \mathrm{E} 01-4.7800 \mathrm{E} 01$ \\
\hline 120 & $1.7600 \mathrm{E} 01-2.9000 \mathrm{E} 01$ \\
\hline 121 & $1.0670 \mathrm{E} 01-1.7600 \mathrm{E} 01$ \\
\hline 122 & $6.4700 \mathrm{E} 00-1.0670 \mathrm{E} 01$ \\
\hline 123 & $3.9300 \mathrm{E} 00-6.4700 \mathrm{E} 00$ \\
\hline 124 & $2.3800 \mathrm{E} 00-3.9300 \mathrm{E} 00$ \\
\hline 125 & $1.4400 \mathrm{E} 00-2.3800 \mathrm{E} 00$ \\
\hline 126 & $8.7600 \mathrm{E}-01-1.4400 \mathrm{E} 00$ \\
\hline 127 & $5.3100 \mathrm{E}-01-8.7600 \mathrm{E}-01$ \\
\hline 128 & $4.1400 \mathrm{E}-01-5.3100 \mathrm{E}-01$ \\
\hline 129 & $1.0000 \mathrm{E}-04-4.1400 \mathrm{E}-01$ \\
\hline
\end{tabular}

\begin{tabular}{r} 
Lethargy Range \\
\hline $4.934-4.991$ \\
$4.991-5.500$ \\
$5.500-5.655$ \\
$5.655-5.809$ \\
$5.809-5.915$ \\
$5.915-5.991$ \\
$5.991-6.075$ \\
$6.075-6.250$ \\
$6.250-6.500$ \\
$6.500-7.001$ \\
$7.001-7.500$ \\
$7.500-8.001$ \\
$8.001-8.502$ \\
$8.502-9.003$ \\
$9.003-9.250$ \\
$9.250-9.499$ \\
$9.499-9.750$ \\
$9.750-10.252$ \\
$10.252-10.501$ \\
$10.501-11.000$ \\
$11.000-11.503$ \\
$11.503-11.750$ \\
$11.750-12.251$ \\
$12.251-12.751$ \\
$12.751-13.250$ \\
$13.250-13.751$ \\
$13.751-14.251$ \\
$14.251-14.749$ \\
$14.749-15.251$ \\
$15.251-15.753$ \\
$15.753-16.250$ \\
$16.250-16.751$ \\
$16.751-17.000$ \\
$17.000-25.328$
\end{tabular}


Photon Interaction Cross Sections

\begin{tabular}{ccc} 
Group & Group Limits (MeV) \\
\hline 1 & $1.00000 \mathrm{E} 01$ & $1.40000 \mathrm{E} 01$ \\
2 & $9.00000 \mathrm{E} 00$ & $1.00000 \mathrm{E} 01$ \\
3 & $8.00000 \mathrm{E} 00$ & $9.00000 \mathrm{E} 00$ \\
4 & $7.76000 \mathrm{E} 00$ & $8.00000 \mathrm{E} 00$ \\
5 & $7.72000 \mathrm{E} 00$ & $7.76000 \mathrm{E} 00$ \\
6 & $7.50000 \mathrm{E} 00$ & $7.72000 \mathrm{E} 00$ \\
7 & $7.04000 \mathrm{E} 00$ & $7.50000 \mathrm{E} 00$ \\
8 & $7.02000 \mathrm{E} 00$ & $7.04000 \mathrm{E} 00$ \\
9 & $6.73000 \mathrm{E} 00$ & $7.02000 \mathrm{E} 00$ \\
10 & $6.71000 \mathrm{E} 00$ & $6.73000 \mathrm{E} 00$ \\
11 & $6.14000 \mathrm{E} 00$ & $6.71000 \mathrm{E} 00$ \\
12 & $6.12000 \mathrm{E} 00$ & $6.14000 \mathrm{E} 00$ \\
13 & $5.84000 \mathrm{E} 00$ & $6.12000 \mathrm{E} 00$ \\
14 & $5.82000 \mathrm{E} 00$ & $5.84000 \mathrm{E} 00$ \\
15 & $5.12000 \mathrm{E} 00$ & $5.82000 \mathrm{E} 00$ \\
16 & $5.09500 \mathrm{E} 00$ & $5.12000 \mathrm{E} 00$ \\
17 & $4.45000 \mathrm{E} 00$ & $5.09500 \mathrm{E} 00$ \\
18 & $4.43000 \mathrm{E} 00$ & $4.45000 \mathrm{E} 00$ \\
19 & $3.86000 \mathrm{E} 00$ & $4.43000 \mathrm{E} 00$ \\
20 & $3.84000 \mathrm{E} 00$ & $3.86000 \mathrm{E} 00$ \\
21 & $3.69000 \mathrm{E} 00$ & $3.84000 \mathrm{E} 00$ \\
22 & $3.67000 \mathrm{E} 00$ & $3.69000 \mathrm{E} 00$ \\
23 & $3.38000 \mathrm{E} 00$ & $3.67000 \mathrm{E} 00$ \\
24 & $3.36000 \mathrm{E} 00$ & $3.38000 \mathrm{E} 00$ \\
25 & $2.84000 \mathrm{E} 00$ & $3.36000 \mathrm{E} 00$ \\
26 & $2.82000 \mathrm{E} 00$ & $2.84000 \mathrm{E} 00$ \\
27 & $2.80000 \mathrm{E} 00$ & $2.82000 \mathrm{E} 00$ \\
28 & $2.78000 \mathrm{E} 00$ & $2.80000 \mathrm{E} 00$ \\
29 & $2.32000 \mathrm{E} 00$ & $2.78000 \mathrm{E} 00$ \\
30 & $2.30000 \mathrm{E} 00$ & $2.32000 \mathrm{E} 00$ \\
31 & $2.13000 \mathrm{E} 00$ & $2.30000 \mathrm{E} 00$ \\
32 & $2.11000 \mathrm{E} 00$ & $2.13000 \mathrm{E} 00$ \\
33 & $1.79000 \mathrm{E} 00$ & $2.11000 \mathrm{E} 00$
\end{tabular}

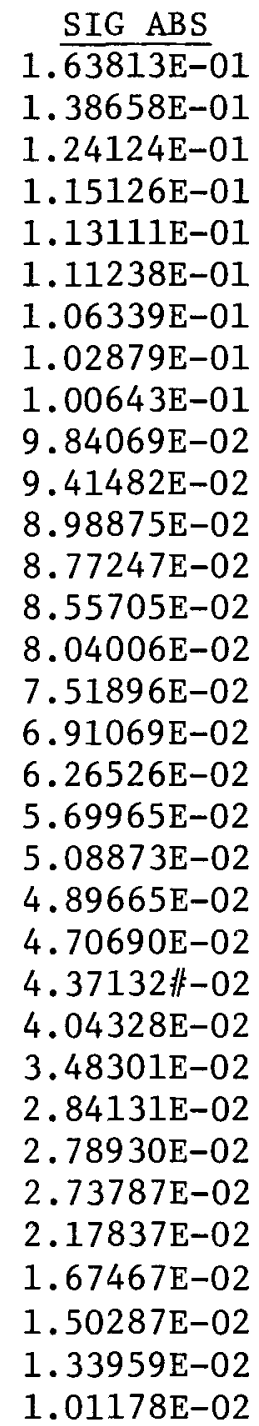

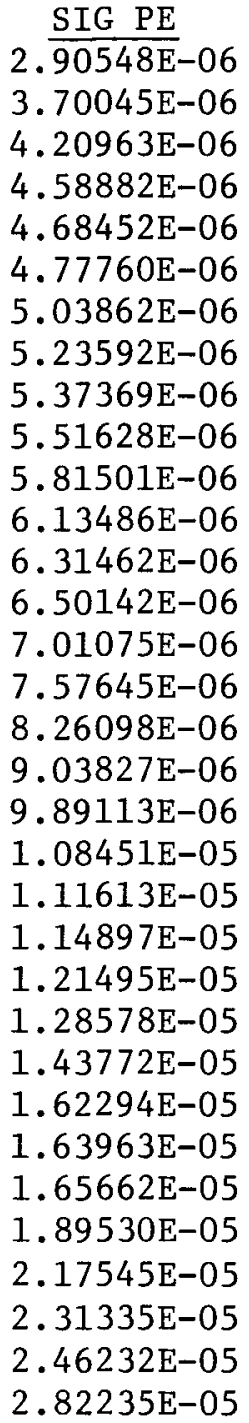

$\begin{array}{cc}\text { SIG PP } & \text { ENERGY DEP } \\ 1.63810 \mathrm{E}-01 & 4.78338 \mathrm{E} 00 \\ 1.38654 \mathrm{E}-01 & 3.91056 \mathrm{E} 00 \\ 1.24119 \mathrm{E}-01 & 3.54783 \mathrm{E} 00 \\ 1.15122 \mathrm{E}-01 & 3.33044 \mathrm{E} 00 \\ 1.13106 \mathrm{E}-01 & 3.28231 \mathrm{E} 00 \\ 1.11233 \mathrm{E}-01 & 3.23790 \mathrm{E} 00 \\ 1.06334 \mathrm{E}-01 & 3.12268 \mathrm{E} 00 \\ 1.02874 \mathrm{E}-01 & 3.04195 \mathrm{E} 00 \\ 1.00638 \mathrm{E}-01 & 2.99020 \mathrm{E} 00 \\ 9.84014 \mathrm{E}-02 & 2.93860 \mathrm{E} 00 \\ 9.41424 \mathrm{E}-02 & 2.84104 \mathrm{E} 00 \\ 8.98814 \mathrm{E}-02 & 2.74386 \mathrm{E} 00 \\ 8.77185 \mathrm{E}-02 & 2.69467 \mathrm{E} 00 \\ 8.55640 \mathrm{E}-02 & 2.64558 \mathrm{E} 00 \\ 8.03937 \mathrm{E}-02 & 2.52788 \mathrm{E} 00 \\ 7.51920 \mathrm{E}-02 & 2.40924 \mathrm{E} 00 \\ 6.90987 \mathrm{E}-02 & 2.29427 \mathrm{E} 00 \\ 6.26436 \mathrm{E}-02 & 2.17853 \mathrm{E} 00 \\ 5.69866 \mathrm{E}-02 & 2.07562 \mathrm{E} 00 \\ 5.08765 \mathrm{E}-02 & 1.97083 \mathrm{E} 00 \\ 4.89553 \mathrm{E}-02 & 1.93997 \mathrm{E} 00 \\ 4.70575 \mathrm{E}-02 & 1.90912 \mathrm{E} 00 \\ 4.37011 \mathrm{E}-02 & 1.85277 \mathrm{E} 00 \\ 4.04199 \mathrm{E}-02 & 1.79629 \mathrm{E} 00 \\ 3.48158 \mathrm{E}-02 & 1.69617 \mathrm{E} 00 \\ 2.83969 \mathrm{E}-02 & 1.59281 \mathrm{E} 00 \\ 2.78766 \mathrm{E}-02 & 1.58494 \mathrm{E} 00 \\ 2.73622 \mathrm{E}-02 & 1.57707 \mathrm{E} 00 \\ 2.17647 \mathrm{E}-02 & 1.48170 \mathrm{E} 00 \\ 1.67250 \mathrm{E}-02 & 1.38491 \mathrm{E} 00 \\ 1.50056 \mathrm{E}-02 & 1.34548 \mathrm{E} 00 \\ 1.33713 \mathrm{E}-02 & 1.30559 \mathrm{E} 00 \\ 1.00896 \mathrm{E}-02 & 1.23102 \mathrm{E} 00\end{array}$


Photon Interaction Cross Sections (Cont'd.)

\begin{tabular}{lll} 
Group & \multicolumn{3}{c}{ Group Limits $(\mathrm{MeV})$} \\
\hline 34 & $1.77000 \mathrm{E} 00$ & $1.79000 \mathrm{E} 00$ \\
35 & $1.64000 \mathrm{E} 00$ & $1.77000 \mathrm{E} 00$ \\
36 & $1.62000 \mathrm{E} 00$ & $1.64000 \mathrm{E} 00$ \\
37 & $7.38000 \mathrm{E}-01$ & $1.62000 \mathrm{E} 00$ \\
38 & $7.18000 \mathrm{E}-01$ & $7.38000 \mathrm{E}-01$ \\
39 & $5.20000 \mathrm{E}-01$ & $7.18000 \mathrm{E}-01$ \\
40 & $5.00000 \mathrm{E}-01$ & $5.20000 \mathrm{E}-01$ \\
41 & $3.00000 \mathrm{E}-01$ & $5.00000 \mathrm{E}-01$ \\
42 & $1.00000 \mathrm{E}-01$ & $3.00000 \mathrm{E}-01$ \\
43 & $1.00000 \mathrm{E}-04$ & $1.00000 \mathrm{E}-01$
\end{tabular}

SIG ABS
$6.53062 \mathrm{E}-03$
$5.32255 \mathrm{E}-03$
$4.26155 \mathrm{E}-03$
$7.67590 \mathrm{E}-04$
$2.08687 \mathrm{E}-04$
$3.39119 \mathrm{E}-04$
$5.54766 \mathrm{E}-04$
$1.25894 \mathrm{E}-03$
$1.64216 \mathrm{E}-02$
$6.08122 \mathrm{E} 02$

SIG PE
$3.28530 \mathrm{E}-05$
$3.54684 \mathrm{E}-05$
$3.83345 \mathrm{E}-05$
$8.15626 \mathrm{E}-05$
$2.08687 \mathrm{E}-04$
$3.39119 \mathrm{E}-04$
$5.54766 \mathrm{E}-04$
$1.25894 \mathrm{E}-03$
$1.64216 \mathrm{E}-02$
$6.08122 \mathrm{E} 02$

SIG PP
$6.49777 \mathrm{E}-03$
$5.28709 \mathrm{E}-03$
$4.22322 \mathrm{E}-03$
$6.86027 \mathrm{E}-04$
0.0
0.0
0.0
0.0
0.0
0.0

ENERGY DEP

$1.15399 \mathrm{E} 00$

$1.11890 \mathrm{E} 00$

$1.08330 \mathrm{E} 00$

$8.40891 \mathrm{E}-01$

$5.64813 \mathrm{E}-01$

$4.85497 \mathrm{E}-01$

$4.02880 \mathrm{E}-01$

$3.13480 \mathrm{E}-01$

$1.43309 \mathrm{E}-01$

$1.71055 \mathrm{E} 00$ 
-

$?$ 


\section{Internal Distribution}

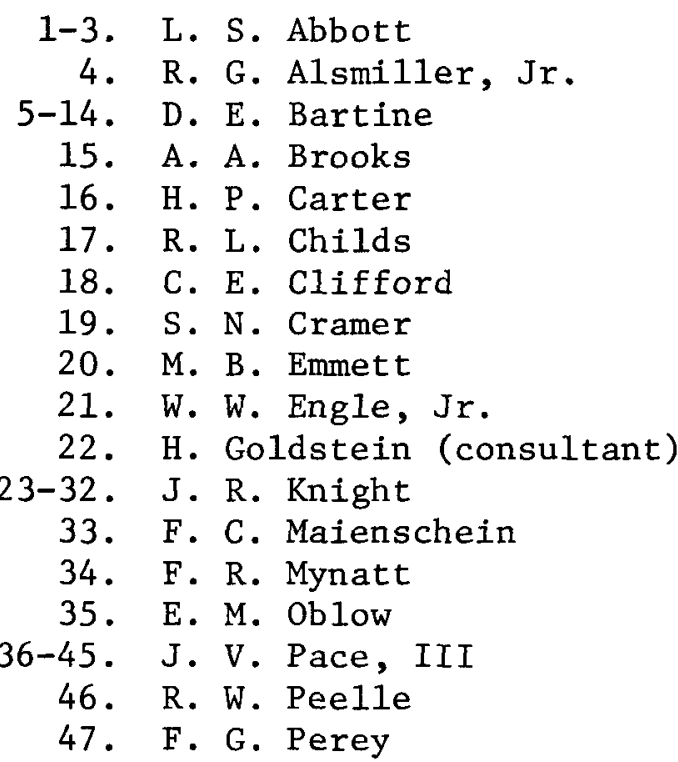

48. R. W. Roussin

49. C. O. Slater

50. D. K. Trubey

51. C. R. Weisbin

52. R. M. Westfall

53. G. E. Whitesides

54. A. Zucker

55. Paul Greebler (consultant)

56. W. W. Havens, Jr. (consultant)

57. A. F. Henry (consultant)

58. R. E. Uhrig (consultant)

59-60. Central Research Library

61. ORNL Y-12 Technical Library,

Document Reference Section

62-63. Laboratory Records Department

64. Laboratory Records ORNL RC

65. ORNL Patent Office

66. Computer Science Division Library

67-116. RSIC

External Distribution

117. P. B. Hemmig, Division of Reactor Research and Development, Energy Research and Development Administration, Washington, D. C. 20545

118. Research and Technical Support Division, Energy Research and Development Administration, Oak Ridge, Tennessee 37830

119-191. Given DNA Radiation Transport Distribution

192-218. Technical Information Center, ORO

219. Dr. David Auton, Defense Nuclear Agency, Attn: RATN, Tactical Nuclear Division, Washington, D. C. 20305

220. Norm Banks, Ballistics Research Lab., Aberdeen Proving Ground, Maryland 21005

221. J. Burgio, Air Force Weapons Lab., Albuquerque, N.M. 87117

222. V. R. Cain, SAI, 114 Tulsa Rd., Oak Ridge, Tenn. 37830

223. Tom DeRosa, Picatinny Arsena1, NDED B1dg. 65, Dover, New Jersey 07840

224. Steve Dupree, Organization 5231, Sandia Labs., Albuquerque, N.M. 08715

225. J. Fasano, Picatinny Arsenal, NDED B1dg. 65, Dover, New Jersey 07840

226. Dr. D. Kaul, 305 Kinsingston Ct., Palatine, Illinois 60067

227. Ltc. R. M. Roberds, Attn: AFWL/DYT, Kirtland Air Force Base, N.M. 87117

228. R. A. Shulstad, Air Force Weapons Lab., Albuquerque, N.M. 87117

229. Capt. A. S. Warshawsky, Department of the Army, Headquarters, United States Army Nuclear Agency, Fort Bliss, Texas 79916 